Aus der Abteilung Prothetik

(Prof. Dr. med. Dr. med. dent. A. Hüls)

im Zentrum Zahn-, Mund- und Kieferheilkunde

der Medizinischen Fakultät der Universität Göttingen

\title{
Retrospektive klinische Studie zum Langzeitverhalten von In-Ceram-Einzelzahnkronen über einen Zeitraum von mehr als 10 Jahren
}

\author{
INAUGURAL - DISSERTATION \\ zur Erlangung des Doktorgrades \\ für Zahnheilkunde \\ der Medizinischen Fakultät \\ der Georg-August-Universität zu Göttingen
}

vorgelegt von

Athanasios Tsigaras

aus Bielefeld 
Dekan:

Prof. Dr. med. C. Frömmel

I. Berichterstatter:

II. Berichterstatter/in:

\section{Berichterstatter/in:}

Tag der mündlichen Prüfung:

Prof. Dr. med. Dr. med. dent. A. Hüls 


\section{Inhaltsverzeichnis}

2.1 Dentalkeramik $\quad 8$

2.1.1 Historischer Rückblick $\quad 8$

2.1.2 Dentalkeramische Massen 9

2.1.3 Systematik keramischer Systeme 9

$\begin{array}{lll}2.2 & \text { Vollkeramische Versorgungen } & 10\end{array}$

2.3 Hochleistungskeramiken auf der Basis von Metalloxiden 11

2.3.1 Das In-Ceram-System 11

2.3.2 Aluminiumoxid - Infiltrationskeramiken 11

$\begin{array}{lll}2.3 .3 & \text { Zirkoniumdioxid } & 13\end{array}$

2.3.4 Klinische Erfahrungen mit In-Ceram 14

2.4 VMK-Kronen 16

2.4.1 Überblick 16

2.4.2 Vorteile 16

$\begin{array}{lll}2.4 .3 & \text { Nachteile } & 16\end{array}$

2.4.4 Langzeitverhalten/Verweildauer 16

2.5 Gründe für den Verlust von Restaurationen 17

$\begin{array}{lll}2.5 .1 & \text { Sekundärkaries } & 17\end{array}$

2.5.2 Vitalitätsverluste und endodontische Komplikationen 17

$\begin{array}{lll}2.5 .3 \text { Zahnextraktionen } & 19\end{array}$

$\begin{array}{lll}2.5 .4 & \text { Keramikfrakturen } & 20\end{array}$

$3 \quad$ Material und Methoden $\quad 22$

3.1 Patientengut 22

3.1.1 Systematische Aktenanalyse 22

3.1.2 Klinische Nachuntersuchung 22 
3.2 Untersuchungsablauf 23

3.3 Statistische Analyse 26

$\begin{array}{llr}4 & \text { Ergebnisse } & 27\end{array}$

4.1 Charakterisierung des Untersuchungsgutes 27

$\begin{array}{lll}\text { 4.1.1 Das Patientengut } & 27\end{array}$

4.1.1.1 Geschlechtsverteilung 27

4.1.1.2 Mundhygienestatus 28

4.1.1.3 Häufigkeitsverteilung der Restaurationen pro Patient 29

4.1.2 Die Restaurationen 30

4.1.2.1 Verteilung der Restaurationen bezogen auf die einzelnen Zähne (FDI) 30

4.1.2.2 Art der Zementierung 31

4.1.2.3 Endodontische Vorbehandlung der Pfeilerzähne 31

4.1.2.4 Liegezeiten der Restaurationen $\quad 32$

4.2 Ergebnisse der Nachuntersuchung 32

4.2.1 15-Jahres-Ergebnisse 32

4.2.1.1 Charakteristika der Restaurationen 32

4.2.1.2 Überlebenswahrscheinlichkeit (in-situ-Kriterium) 33

4.2.1.3 Erfolgswahrscheinlichkeit (ereignisfreie Restaurationen) 34

$\begin{array}{lll}\text { 4.2.2 10-Jahres-Ergebnisse } & 35\end{array}$

5 Diskussion $\quad 53$

$\begin{array}{lll}6 & \text { Schlussfolgerungen } & 61\end{array}$

$\begin{array}{lll}7 & \text { Zusammenfassung } & 62\end{array}$

$8 \quad$ Literaturverzeichnis $\quad 63$ 
9.1 Protokoll für die Nachuntersuchung CRF 72

9.2 Votum der Ethikkommission $\quad 75$

9.3 Aufklärungsbogen Patient $\quad 76$

9.4 Einverständniserklärung Patient $\quad 78$

9.5 Publikationen im Zusammenhang mit dieser Dissertation 79

$\begin{array}{lll}\text { 9.5.1 Postervortrag } & 79\end{array}$

9.5.2 Wissenschaftliches Journal 79 


\section{$1 \quad$ Einleitung}

Diskussionen über die Amalgamproblematik, ein hohes Maß an Gesundheitsbewusstsein und nicht zuletzt das Bild des Schönheitsideales in der breiten Bevölkerung haben zur Entwicklung einer Vielzahl neuer biokompatibler, ästhetisch anspruchsvoller Restaurationsmethoden und Materialien geführt (Pröbster, 1997; Adamcyk und Spiechowicz, 1990). Die Zahl der Eingliederungen von vollkeramischen Inlays, Onlays, Kronen und Brücken ist in den letzten zehn Jahren kontinuierlich auf über 5,5 Millionen Restaurationen pro Jahr gestiegen und erzielte damit einen Anteil von 20\% am Behandlungsvolumen, das für zahnerhaltende und prothetische Versorgungen indiziert ist. Diese Entwicklung wurde gefördert durch die Verfügbarkeit hochfester Restaurationskeramiken sowie durch die CAD/CAM-Technik und Software mit zahntechnischen Applikationen (Kern, 2010).

Dentale Keramiken werden hohen ästhetischen Ansprüchen gerecht, die Haltbarkeit ist gewährleistet, die physikalischen und chemischen Eigenschaften sind weitgehend untersucht und die Adhäsion von Mikroorganismen an ihren Oberflächen ist teilweise niedriger als auf natürlichen Zähnen (Chan und Weber, 1986). Die Struktur akkumulierter Plaque ist im Vergleich zu anderen Materialien weniger dicht, weshalb vollkeramische Restaurationen als parodontalfreundlich eingestuft werden können (Adamcyk und Spiechowicz, 1990; Schwickerath, 1987).

Unverträglichkeitsreaktionen wie Allergien oder lokaltoxische Reaktionen sind zwar bei hochwertigen und korrosionsresistenten Legierungen außerordentlich selten, dennoch verlangen diese Einzelfälle nach therapeutischen Alternativen, die zumindest teilweise mit keramischen Werkstoffen zu realisieren sind (Kappert et al., 1994; Strub et al., 1999).

Die Eingliederung vollkeramischer Restaurationen wurde wegen deren mechanischer Stabilität jedoch häufig kontrovers diskutiert (Erpenstein und Kerschbaum, 1995). Neue Dentalkeramiken mit den unterschiedlichsten kristallinen Inhaltsstoffen wurden entwickelt, um die Rissausbreitung bei Zugbelastung positiv $\mathrm{zu}$ beeinflussen und damit höhere Bruchfestigkeitswerte als bei herkömmlichen Keramiken $\mathrm{zu}$ garantieren. In diesem Zusammenhang sind vor allem auch die hartkernbasierten Keramiken wie glas-infiltrierte Oxidkeramiken (Bsp.: In-Ceram) und Zirkoniumdioxid zu nennen. Aufgrund der spezifischen verbesserten mechanischen Eigenschaften dieser hochfesten Strukturkeramiken konnten Indikationsbereiche wie Kronen und sogar Brücken im Seitenzahngebiet erschlossen werden, welche bei Versorgung mittels konventioneller Feldspat- oder Glaskeramiken erhöhte Misserfolgsraten zeigten (Pospiech, 2007). 
Eine multizentrische Studie der AG Keramik (Leitung: Dr. Reiss, Malsch) beinhaltet die Bewertung von 5.700 vollkeramischer Restaurationen aus über 200 privaten Praxen mit dem Ziel einer Qualitätssicherung im relativen Praxisvergleich untereinander. In diesem Zusammenhang werden Überlebensraten verschiedener vollkeramischer Restaurationen von 83\% nach einer mittleren Beobachtungszeit von 13 Jahren beschrieben (Kern, 2010). 


\section{$2 \quad$ Literaturübersicht}

\subsection{Dentalkeramik}

\subsubsection{Historischer Rückblick}

Keramiken umfassen eine große Familie anorganischer Stoffe innerhalb der Werkstoffgruppe der Nichtmetalle. Der Name „Keramik“ kommt aus dem Griechischen und ist mit den Worten ,gebrannter Stoff“, der aus Tonerde gefertigt wird, zu übersetzen (Friedmann, 1991). In China war man bereits vor rund zweieinhalbtausend Jahren mit der Herstellung von Porzellan vertraut. Johann F. Böttger, Gründer der Meissener Porzellanmanufaktur, versuchte schon im frühen 17. Jahrhundert vergeblich, die Kunst der Porzellanherstellung der Chinesen zu kopieren.

Die französischen Apotheker Alexis Duchateau und de Charmant waren die ersten, die aus Porzellan gefertigte Vollprothesen herstellten. Erst durch den italienischen Dentisten Giuseppe Fonzi (1768-1840), der den Weg für eine Serienproduktion wesentlich prägte, wurde die Herstellung von Keramikzähnen entscheidend weiterentwickelt. Schon damals gelang es ihm, Porzellanzähne mit Hilfe eingebrannter Metallstifte mechanisch im Prothesenkörper zu verankern. Die Fertigung von Keramikfrontzähnen nach dem heutigen Solila-Prinzip basiert auf dem Prinzip der damaligen Entdeckung (Strub et al., 1999). Dabei werden zunächst zwei kleine geschlitzte Edelmetallhülsen direkt in den Kern des Keramikzahns eingebrannt, um sie anschließend mit Hilfe eines Lotplättchens an einen knopfförmig verdickten Goldmantelstift im Durchlaufofen anzulöten.

Die ersten Zahnfabriken entstanden Ende des 17. Jahrhunderts in den USA, wobei die bekanntesten Brennereien die von Peale und Stockton waren. 100 Jahre später gründete Friedrich August Wienand die erste deutsche Zahnfabrik in Pforzheim, die heute zur Firma De Trey/Dentsply gehört.

Bereits 1989 brachte VITA die erste Materialvariante des Vollkeramik-Systems VITA In-Ceram - VITA In-Ceram ALUMINA -auf den Markt. Seitdem entwickelte das Dentalunternehmen immer wieder neue, innovative Varianten dieses Materials. Bis heute können Zahntechniker und Zahnärzte aus insgesamt fünf Materialvarianten in unterschiedlichsten Aggregatzuständen für diverse Verarbeitungsvarianten - vom Powder für die Schlickertechnik bis zu Blöcken für die CAD/CAM-gestützte Herstellung von Vollkeramik-Restaurationen - wählen. Seit 1930 stellt die VITA- 
Zahnfabrik Materialien für vollkeramische zahnärztliche Restaurationen her, von den historischen VITA-LUMIN- und VITADUR-Keramiken bis zu den VITA-In-Ceram Sinterkeramiken (Pröbster und Groten, 2006).

\subsubsection{Dentalkeramische Massen}

Dentalkeramiken, zu denen auch die Feldspatkeramiken zählen, entsprachen zu Beginn ihrer Entwicklung etwa der Zusammensetzung des chinesischen Porzellans. Der Anteil von Kaolin lag ungefähr bei 40-70\%, der von Quarz und Feldspat lediglich zwischen 15-30\% (Marx und Meiners, 2001). Aus diesen drei Grundstoffen entstehen beim Brennen Leucit und Mullit, die sich durch eine unerwünschte Weißfärbung und nur geringe Transluzenz auszeichnen. Deshalb wurde der Feldspatanteil zu Lasten des Kaolinanteils erhöht, wodurch die Transluzenz der Keramik entscheidend verbessert werden konnte (McLean, 1978). Heutige moderne Verblendkeramiken enthalten fast ausschließlich Feldspat (65-90\%), 10-30\% Quarz und nur noch 0-5\% Kaolin (Pospiech, 2007).

\subsubsection{Systematik keramischer Systeme}

Keramiken umfassen ein breites werkstoffkundliches Spektrum. Man unterscheidet u.a Silikatkeramiken, Oxidkeramiken und nichtoxidische Keramiken (Schüller und Hennicken, 1985).

Hauptbestandteil der Silikatkeramiken ist eine glasig amorphe Matrixmasse (Glasphase) aus Siliziumoxid $\left(\mathrm{SiO}_{2}\right)$ mit ausgeprägter Porenstruktur, in die eine Kristallphase eingelagert ist. Ihr Grundbaustein ist ein Siliziumatom, tetraedrisch umgeben von vier Sauerstoffatomen, die über ionische und kovalente Bindungen Kristallgitter bilden.

Im Unterschied zu Verblendkeramiken weisen oxidkeramische Werkstoffe vor allem eine kristalline Struktur auf, während ihr Anteil an amorphen Glasbestandteilen zu vernachlässigen ist. Zu den einfachen Oxidkeramiken gehören die Keramiken aus der Stoffgruppe der Spinelle. Nichtoxidische Keramiken (z.B. Carbide, Nitride) sind im Allgemeinen schwarz und daher zur Aufbereitung für Dentalkeramiken ungeeignet (Hahn und Löst, 1992). Oxidkeramiken bieten aufgrund ihrer physiko-chemischen Eigenschaften und unterschiedlichen Herstellungsvarianten ein besonders breites 
klinisches Anwendungsspektrum unter Einbeziehung größerer Brückenkonstruktionen (Pospiech, 2007).

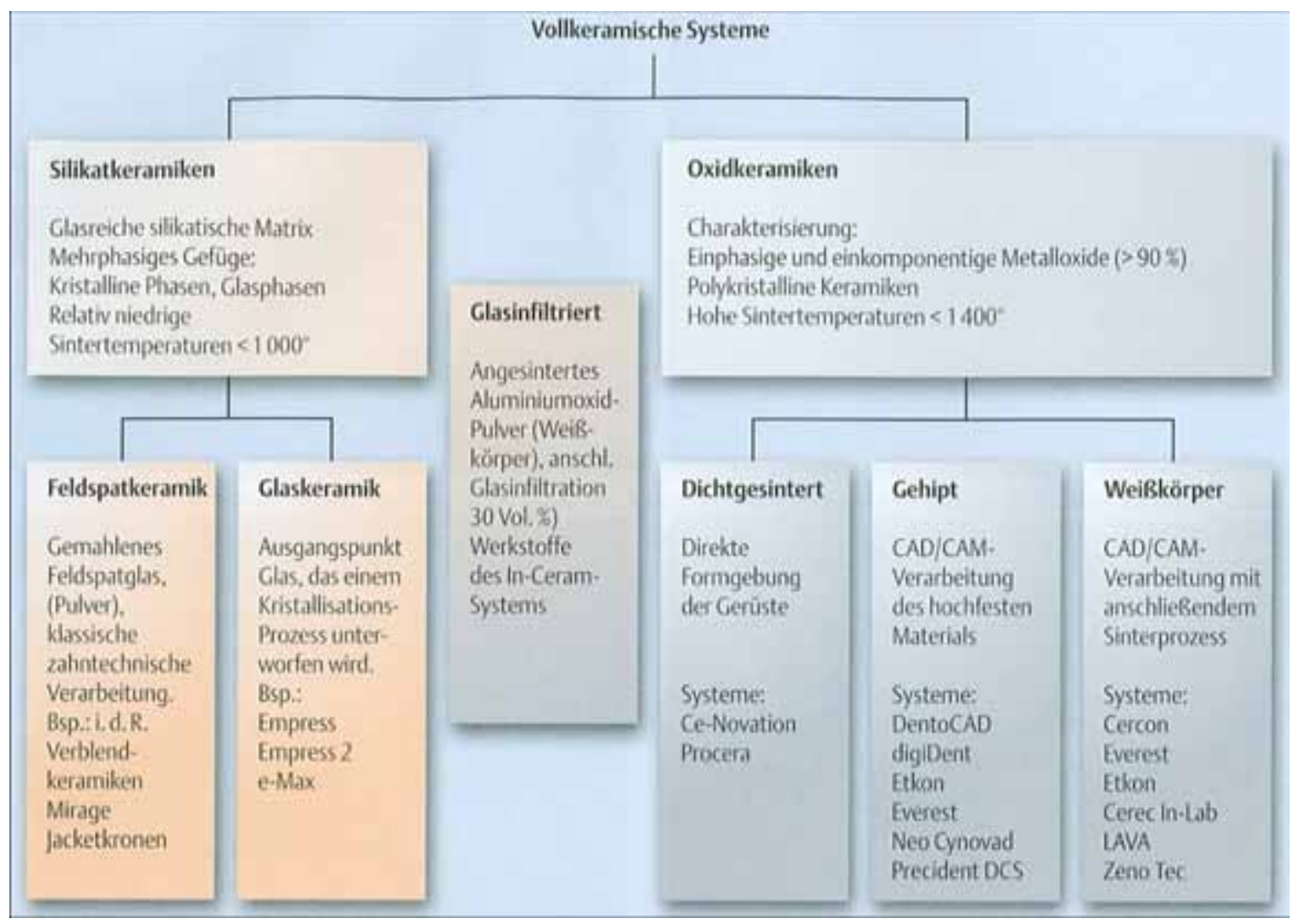

Abbildung 1: Überblick über aktuelle vollkeramische Systeme unter werkstoffkundlichen Aspekten. (aus: Pospiech, 2007, S.72)

\subsection{Vollkeramische Versorgungen}

Für die Einzelzahnrestaurationen bieten sich abhängig vom Zerstörungsgrad verschiedene Versorgungsformen an.

Veneers werden häufig aus ästhetischen Gründen und wegen der schmelzähnlicheren Eigenschaften als sinnvolle Alternative zu ausgedehnten Kunststofffüllungen gesehen. Wie für die Veneers gilt gleiches auch für die Inlay-/Onlayrestaurationen im Seitenzahnbereich. Für die okklusionstragende Rekonstruktion ist die Keramik das Mittel der Wahl, da sie gegenüber vereinzelt diskutierten Kompositrestaurationen den Vorteil der schmelzähnlichen Abrasionswerte hat und aufgrund ihrer anorganischen Apatitstruktur den Zahnschmelzstrukturen am nächsten kommt. 
Für den Frontzahnbereich kommen alle Keramiken für die Restaurationen in Frage. Im Prämolaren- und Molarenbereich sollten eher die Glaskeramiken der 2. Generation oder verblendete Oxidkeramiken verwendet werden.

Auch zum Ersatz verloren gegangener Zähne besteht die Möglichkeit, vollkeramische Restaurationen einzugliedern. Neben der vollkeramischen Krone auf einem Implantat mit vollkeramischem Abutment ist die konventionelle Versorgungsform mittels Anfertigung einer Brücke auf der Basis eines Gerüstes aus Hochleistungskeramik (Zirkoniumdioxid) und einer Verblendung mit Glaskeramik ebenso denkbar. Die Versorgung mit vollkeramischen Brücken mit bis zu zwei Zwischengliedern ist möglich. Hier liegen klinische Daten über mehr als fünf Jahre vor (Rammelsberg und Ohlmann, 2007).

\subsection{Hochleistungskeramiken auf der Basis von Metalloxiden}

\subsubsection{Das In-Ceram-System}

Die Basis moderner Hochleistungskeramiken bilden Aluminiumoxid $\left(\mathrm{Al}_{2} \mathrm{O}_{3}\right)$, Zirkoniumdioxid $\left(\mathrm{ZrO}_{2}\right)$, Siliciumcarbid $(\mathrm{SiC})$ und Siliciumnitrid $\left(\mathrm{Si}_{3} \mathrm{~N}_{4}\right)$ (Luthardt et al., 1998). In der konservierenden und prothetischen Zahnheilkunde sind jedoch nur Aluminiumoxid und Zirkoniumdioxid von Interesse (Rüde und Evans, 1989).

Die gewünschten indikationsspezifischen Hochleistungseigenschaften können nur durch eine hoch technisierte Fertigung standardisierter Rohlinge (Preforms) realisiert werden. Ziel dieser modernen Werkstoffentwicklung ist die Verringerung der Streuung ihrer Belastbarkeitskennwerte bei gleichzeitiger Steigerung der Gesamtfestigkeit und der Erhöhung ihrer Fehlertoleranz. Grundlage hierfür ist die Steigerung der Bruchzähigkeit, eines bei konventionellen Keramiken kritischen Parameters.

\subsubsection{Aluminiumoxid - Infiltrationskeramiken}

Aluminiumoxid, die Hauptkomponente des In-Ceram-Systems, ist ein natürlich vorkommender Stoff. Aufgrund seiner Härte und seines ungewöhnlich hohen Elastizitätsmoduls von $410 \mathrm{GPa}$ wurde es bereits in früheren Jahren als Schleifmittel und zur Herstellung von hochfesten Materialien verwendet. Seit vielen Jahren wird 
kristallines Aluminiumoxid auch zur Festigkeitssteigerung von Dentalkeramiken, zur so genannten Dispersionsverstärkung, verwendet. Bei dieser Keramik wurde der Aluminiumoxidanteil auf über $80 \%$ erhöht. Dieses neuartige Verfahren entwickelte der Pariser Zahnarzt Michael Sadoun, der damit den Grundstein für die moderne prothetische Vollkeramikversorgung legte, die als In-Ceram-Technologie der Firma Vita seit 1989 auf dem Markt ist (Sadoun, 1988).

Das In-Ceram-Gerüst besteht aus feinstem Aluminiumoxidpulver, das entweder nach Anmischen einer wässrigen Suspension (Schlicker) mit einem Pinsel auf den Gipsstumpf aufgetragen oder mit Hilfe der von Kappert und Knode (1991) entwickelten Gieß- bzw. Spritztechnik hergestellt wird. Das Käppchen wird anschließend im Vita-Inceramat, einem Ofen, der innerhalb von 7,5 Stunden eine Temperatur von $1120^{\circ} \mathrm{C}$ erreicht, gebrannt. Während der zweistündigen Haltezeit kommt es zur Kontraktion des Gipsstumpfes. Gleichzeitig verbacken die Aluminiumoxidkristalle $\mathrm{zu}$ einer kontinuierlichen, porösen, polykristallinen Matrix. Ein Sinterungsprozess, ähnlich dem Brennvorgang einer herkömmlichen Verblendkeramik in der Heißzone des Keramikofens, findet dabei nicht statt (Hofer, 1984). Das bis zu diesem Zeitpunkt noch sehr bruchanfällige, offen poröse $\mathrm{Al}_{2} \mathrm{O}_{3}$ Gerüst erhält erst durch den Infiltrationsbrand seine eigentliche Härte. Durch den bestehenden Kapillarstrom diffundiert ein niedrigvisköses Lanthanglas in den offen porösen Primärkern ein (Sadoun, 1988). Da das $\mathrm{Al}_{2} \mathrm{O}_{3}$-Gerüst einen geringfügig höheren thermischen Ausdehnungskoeffizienten als seine Einzelkomponenten aufweist, kommt es bei seiner Abkühlung zum Aufbau interner Druckspannungen im Glas. Bei Zugbelastungen müssen diese zusätzlich überwunden werden, was ein Versagen der Restauration verzögert (Fischer et al., 1991). Das Hartkerngerüst stellt ein homogenes, fast porenfreies Mikrogefüge dar, das nach Entfernung des Glasüberschusses mit herkömmlichen Keramikmassen verblendet werden kann.

Spinell (Magnesiumaluminat, $\mathrm{MgAl}_{2} \mathrm{O}_{4}$ ) gilt als weitere Modifikation der In-CeramKeramik. Aufgrund seiner hervorragenden Transluzenz und der dem natürlichen Schmelz sehr ähnlichen Lichttransmission, wurde das Indikationsgebiet speziell auf Veneers und Frontzahnkronen erweitert. Allerdings führte diese optische Verbesserung zu einer signifikanten Reduktion der mechanischen Festigkeit, die durch die größere Partikelgröße erklärt werden kann (Seghi et al., 1990). Anders als beim Aluminiumoxid mit seiner hexagonalen Struktur weist die Spinellstruktur eine kubische Anordnung des Atombaus auf, die Doppelbrechungen unmöglich macht 
(Kappert, 1996). Dies erklärt die positiven lichtoptischen Eigenschaften der Spinellkeramik.

\subsubsection{Zirkoniumdioxid}

Bei Zirkoniumdioxid-TZP (Tetragonal Zirkonia Polycristals) handelt es sich um eine industriell gefertigte Hochleistungskeramik mit einer mittleren Korngröße von 0,4 $\mu \mathrm{m}$ (Rieger, 1993). Durch heißisostatisches Pressen feinkörnigen Rohmaterials bei ca. $1500{ }^{\circ} \mathrm{C}$ entsteht ein Industriewerkstoff mit sehr hoher Packungsdichte und nahezu fehlerfreier Qualität (Kappert et al., 1995). Zirkoniumdioxid findet seit vielen Jahren Verwendung in der Orthopädie, speziell im Fachgebiet der Hüftgelenkendoprothetik (Rieger, 1993). In der Zahnmedizin dient es seit langem als Grundlage metallkeramischer Opaquermassen.

Erste Untersuchungen konnten beweisen, dass die Festigkeitseigenschaften der InCeram-Aluminiumoxidkeramik durch die Einlagerung feindisperser Zirkoniumdioxidpartikel in die Aluminiumoxid-Matrix deutlich gesteigert werden können. Abhängig von seiner Wärmebehandlung liegt Zirkoniumdioxid in verschiedenen Modifikationen vor (Maier, 1991). Aus der monoklinen Tieftemperaturphase entsteht bei Temperaturen oberhalb von $1170{ }^{\circ} \mathrm{C}$ durch die martensitische Umwandlung, auch Transformationsverfestigung genannt, die tetragonale Modifikation (Boysen et al., 1991). Durch die Dotierung von Oxiden wie Calciumoxid $(\mathrm{CaO})$, Yttriumoxid $\left(\mathrm{Y}_{2} \mathrm{O}_{3}\right)$ oder $\mathrm{Cer}(\mathrm{III})$-Oxid $\mathrm{Ce}_{2} \mathrm{O}_{3}$ ist jedoch eine gezielte Stabilisierung der tetragonalen Phase bis auf Raumtemperatur möglich (Boysen et al., 1991). Bei niedrigen Konzentrationen dieser Zusätze entsteht teilstabilisiertes Zirkoniumdioxid, so genanntes PSZ (Partially Stabilized Zirkonia). Ist deren Konzentration allerdings höher, führt dies zur Bildung von voll stabilisiertem Zirkoniumdioxid, das die Risszähigkeit des teilstabilisierten $\mathrm{ZrO}_{2}$ bei weitem nicht erreicht (Maier, 1991).

Wird das in der tetragonalen Modifikation stabilisierte Zirkoniumdioxid unter der Einwirkung einer äußeren Belastung im Bereich eines Risses hohen Zugspannungen ausgesetzt, so kann, bedingt durch den verminderten Matrixdruck, wieder eine Umwandlung in die monokline Phase stattfinden. Ein Riss, der sich einem tetragonal strukturierten Zirkoniumkristall nähert, löst eine lokale Volumenexpansion von 3-4 \% als Folge der Phasentransformation in die monokline Phase aus. Durch die Existenz 
lokaler Druckspannungen im Kristall werden die Rissflanken komprimiert, was die Ausbreitung des Risses an seinem Rissgrund erschwert (Maier, 1991).

\subsubsection{Klinische Erfahrungen mit In-Ceram}

In einer von Hüls (1995) veröffentlichen Langzeitstudie mit einem BeobachtungsZeitraum von sechs Jahren wurden insgesamt 335 In-Ceram Kronen untersucht. Bei der jährlichen Kontrolluntersuchung in den ersten drei Jahren lag die Erfolgsquote jeweils oberhalb von 97\%, was die günstigen Überlebensprognosen aus den vorangegangenen in-vitro-Studien bestätigte (Hüls, 1995). Scotti et al. (1995) untersuchten insgesamt 63 In-Ceram-Kronen bei 45 Patienten. Nach einer Beobachtungszeit von maximal vier Jahren waren alle Restaurationen in situ. Lediglich eine Gerüstfraktur konnte festgestellt werden.

Umfangreichere klinische Studien wurden von Sadoun 1996 (in: Kappert, 1996) unternommen, der insgesamt über 6000 In-Ceram-Kronen über einen Zeitraum von 10 Jahren beobachtete. Lediglich $0,4 \%$ der vollkeramischen Restaurationen wiesen Frakturen auf. Detaillierte Angaben zur Untersuchungsmethode wurden jedoch nicht gemacht (Kappert, 1996). Ähnliche positive Ergebnisse lieferte eine von Pröbster (1997) veröffentlichte Kohortenstudie, in der er Einzelkronen im Front- und Seitenzahnbereich über einen Zeitraum von 7 Jahren nachuntersuchte. Im Hinblick auf das Zielkriterium - Fraktur des Hartkerns - lag der Misserfolg nach 6 Jahren unterhalb von 3\%. Weitere Aspekte waren das Auftreten von Karies, Sensibilitätsverluste, sondierbare Randspalten oder Abplatzungen vom Hartkerngerüst. In dieser Hinsicht waren weitaus schlechtere, aber dennoch klinisch akzeptable Ergebnisse zu verzeichnen (63,5\% Überlebensrate nach 6 Jahren). In zwei vorangegangenen Studien imponierte das In-Ceram-Gerüst durch keinen einzigen klinischen Misserfolg (Pröbster, 1993; Pröbster, 1996). Moffa et al. (1988) ermittelten nach einer dreijährigen Funktionsperiode lediglich eine Verlustrate von $3,5 \%$ für den Frontzahnbereich und 11,7\% im Prämolarengebiet. Im hinteren Stützzonenbereich war die Misserfolgsquote jedoch dreimal so hoch (Moffa et al., 1988).

In einer In-vitro-Studie wurde die Dotierung von 10 bis 55 Gew.-\% Zirkoniumdioxid zu manuell bzw. maschinell aufbereitetem Aluminiumoxid zunächst an genormten Prüfplättchen, anschließend an dreigliedrigen Oberkieferseitenzahnbrücken mit Zirkoniumdioxidanteil und Brücken aus In-Ceram-Zirconia untersucht. Mit stetig 
steigendem Mischungsverhältnis kam es zur Zunahme der Belastungsparameter. Die höchste Bruchzähigkeit wurde bei den industriell angemischten Prüfkörpern durch Zusatz von 45-55 Gew.-\% tetragonalem Zirkoniumdioxid ermittelt, die mit einem speziellen Lanthanglas-infiltriert wurden. Im axialen Belastungsversuch konnten für die zirkoniumdioxidverstärkten Brücken (45 Gew.-\%) etwa doppelt so hohe Bruchfestigkeiten $(1498 \pm 155 \mathrm{~N})$ gegenüber denjenigen aus reinem Aluminiumoxid $(655 \pm 239 \mathrm{~N})$ festgestellt werden. Auffällig dagegen verhielten sich die unverblendeten Brücken aus In-Ceram-Zirkonia mit relativ niedriger Dotierung (33 Gew.-\%), Unterschiede waren jedoch zu der Gruppe ohne Transformationsverstärkung statistisch nicht signifikant $(1599 \pm 153 \mathrm{~N})$. Im verblendeten Zustand zeigten beide Materialien identische Bruchergebnisse.

Brückengerüste aus industriell hergestellter Zirkoniumdioxidkeramik, die subtraktiv über ein CAD-CAM-Verfahren bearbeitet wurden, imponierten im axialen Belastungstest durch relativ hohe Bruchfestigkeiten $(1937 \pm 124 \mathrm{~N})$ (Tinschert et al., 1999). Angaben zur Volumenverteilung wurden jedoch nicht gemacht. Nach Verblendung des Hartkerngerüsts wurden $2289 \pm 223$ N gemessen.

Andere Untersuchungen zur Bruchfestigkeit an dreigliedrigen Seitenzahnbrücken, denen 30 Gew.-\% Ceroxid-stabilisiertes tetragonales $\mathrm{ZrO}_{2}$ zugesetzt wurde, ergaben unter statischer Last $2480 \pm 387 \mathrm{~N}$, unter dynamischer Dauerlast wurde ein Maximalwert von $1500 \mathrm{~N}$ ermittelt (Kappert et al., 1995). Verglichen mit herkömmlichen In-Ceram Seitenzahnbrücken (1163 N/750 N) konnten Kappert et al. eine Verdopplung der Bruchfestigkeit unter statischen bzw. dynamischen Prüfbedingungen feststellen, die den Einsatz von teilstabilisiertem Zirkoniumdioxid für die Verwendung im okklusionstragenden Seitenzahnbereich geeignet erscheinen lässt.

Anfangs des Jahres 2000 erfolgte die Freigabe von der Firma Vita, die Keramikrohlinge in Form von Grünkörpern (In-Ceram-Zirconia) zur Herstellung von dreigliedrigen Seitenzahnbrücken auf dem Markt anzubieten. Bei den Grünkörpern handelt es sich um angesintertes Material, welches nach erfolgter Formgebung dicht gesintert wird. 


\section{$2.4 \quad$ VMK-Kronen}

\subsection{1 Überblick}

Keramisch verblendete Metallkronen sind aufgrund der hohen Verschleiß- und Farbbeständigkeit heute die meistverbreitete Kronenform im sichtbaren Bereich.

Keramisch verblendete Kronen bestehen aus einem mindestens 0,3 $\mathrm{mm}$ starkem Metallgerüst, das mit einer opaken Grundmasse $(0,2 \mathrm{~mm})$ und einer Aufbrennkeramik (1,2 mm) überzogen wird. Die zervikalen Ränder bleiben frei von Keramik $(0,2 \mathrm{~mm})$, um die Randadaptation zu erhöhen. In der Regel wird das Metallgerüst gusstechnisch aus einer aufbrennfähigen Edelmetalllegierung oder Nichtedelmetalllegierung auf CoCr-Basis hergestellt (Rammelsberg et al., 2007).

\subsubsection{Vorteile}

Aufgrund der hohen Druckfestigkeit und des zuverlässigen Metall-KeramikVerbundes liefern voll verblendete Kronen gute klinische Ergebnisse. Neben der guten Passgenauigkeit, die durch das Metallgerüst gewährleistet wird, ist die geringe Plaqueakkumulation auf der Keramikoberfläche von Vorteil (Rammelsberg und Ohlmann, 2007). Außerdem bieten VMK-Restaurationen auch bei ungünstigeren Präparationsbedingungen ein breiteres Anwendungsspektrum.

\subsubsection{Nachteile}

$\mathrm{Zu}$ den Nachteilen kann man die eingeschränkte Reparaturmöglichkeit bei Abplatzungen nennen. Auch wenn die Kronenränder unter den Gingivarand verlegt werden, wirkt sich ein Metallrand ästhetisch ungünstig aus. Die durch das Metallgerüst veränderte Lichtbrechung und geblockte Lichtstreuung und -beugung lässt den Randbereich häufig grau erscheinen (Rammelsberg und Ohlmann, 2007).

\subsubsection{Langzeitverhalten/Verweildauer}

Für das seit Jahrzehnten als Standard verwendete System der Verblend- Metallkeramik sind für Einzelkronen mittlere klinische Verweildauern von 95-97\% nach 10 Jahren 
(Walton, 1999) und 78\% nach 20 Jahren (Näpänkangas und Raustia, 2008) evaluiert worden.

In einem meta-analytisch angelegten direkten Vergleich von vollkeramischen und metall-keramischen Einzelzahnkronen zeigten beide Versorgungsarten im Frontzahnbereich ähnliche Überlebensraten $\quad(93-95 \%$ für 5 Jahre $)$. Im Seitenzahnbereich waren die In-Ceram-Restaurationen mit $90 \%$ tendenziell unterlegen (Pjetursson et al., 2007). Auch zeigen aktuelle volkeramische Versorgungen eine im Vergleich zur Metallkeramik erhöhte Rate von „Chipping“, also kohäsiven Frakturen der Verblendkeramik (Pospiech, 2010). Insgesamt sind diese Daten allerdings auf einen 5-Jahres-Zeitraum limitiert. Entsprechend wäre eine retrospektive Evaluation der vollkeramischen Einzelzahnkronen über einen Zeitraum von mehr als 10 Jahren von hoher klinischer Relevanz.

\subsection{Gründe für den Verlust von Restaurationen}

\subsubsection{Sekundärkaries}

Unter Sekundärkaries versteht man kariöse Defekte im Randbereich nach Neuversorgung mit zahnärztlichen Restaurationen, welche meistens klinisch und/oder röntgenologisch $\mathrm{zu}$ diagnostizieren sind. Häufigste Ursachen sind über- und unterkonturierte Restaurationen sowie Randspalten zwischen Restauration und Zahnhartsubstanz mit nachfolgender Plaquebildung (sekundäre Kariesprädilektionsstellen). Dabei entsteht eine äußere Läsion, die alle histologischen Merkmale einer beginnenden Karies aufweist. Ist ein Randspalt vorhanden, entsteht eine Kavitätenwandläsion (Hellwig et al., 1999).

2.5.2 Vitalitätsverluste und endodontische Komplikationen

$\mathrm{Zu}$ Vitalitätsverlusten kann als Konsequenz einer partiellen Entzündung in umschriebenen Bereichen oder in der gesamten Kronen- und Wurzelpulpa kommen. Ein Vitalitätsverlust der Pulpa kann nicht nur entzündungsbedingt entstehen, sondern auch dann, wenn aus traumatischen Gründen die Blutversorgung unterbrochen wird. 
Entzündungen der Pulpa können durch zahlreiche natürliche oder iatrogene Ursachen ausgelöst werden. Aus klinisch-praktischen Gründen bietet sich folgende grobe Klassifizierung an:

- infektiöse Pulpitis

- traumatische Pulpitis

- iatrogene Pulpitis.

Zahnärztliche präparative oder restaurative Maßnahmen, die an vitalen Zähnen vorgenommen werden, können eine mehr oder weniger große Irritation der Pulpa verursachen. Eine Einschätzung der ursächlichen Wirkung ist sehr schwer, da die behandelten Zähne zumeist in einem diagnostisch nicht verifizierbaren Maß durch eine Karies vorgeschädigt sind.

Die bei zahnärztlichen Maßnahmen auftretenden Irritationen sind grundsätzlich physikalischer und/oder chemischer Natur.

$\mathrm{Zu}$ den physikalischen Ursachen gehören:

- präparatorische Maßnahmen

- Trocknung der Kavität

- diagnostische Applikation von Kälte oder Wärme

-mechanisches Einbringen von Füllungsmaterialien und Abformungen.

$\mathrm{Zu}$ den chemischen Ursachen zählt die Anwendung von Reinigungs-, Desinfektionsund Trocknungsmitteln, Lacken, Linern, Unterfüllungsmaterialien, Füllungsmaterialien, Säuren und Agenzien zur Ausbildung eines adhäsiven Verbundes.

Besonders häufig entstehen Schädigungen der Pulpa bei der Kronen- und Kavitätenpräparation. Schädigend wirken können hierbei:

- Vibration

- Druck

- Temperaturerhöhung

- Austrocknung des Dentins.

Bei der Präparation des Dentins werden oft großflächig Dentinkanälchen eröffnet. Durch die Trennung der Odontoblastenfortsätze in diesen Kanälen können Entzündungsmediatoren freigesetzt werden, die eine vaskuläre Reaktion in der Pulpa auslösen (Hellwig et al., 1999).

Ungenügende Wasserkühlung bei der Präparation führt $\mathrm{zu}$ Überhitzung und Austrocknung des Dentins. Da Dentin ein schlechter Temperaturleiter ist, ist die 
Temperaturänderung an der pulpalen Dentinwand in Abhängigkeit von der verbleibenden Stärke wesentlich geringer als die von außen einwirkende Temperatur. Bei trockener Kavitätenpräparation kann es in der Pulpa zu einer Temperaturerhöhung von $2-3^{\circ} \mathrm{C}$ kommen, die aber alleine für sich nicht schädigend sein muss. Nachweislich führt erst ein Temperaturanstieg in der Pulpa von $10^{\circ} \mathrm{C} \mathrm{zu}$ einer irreversiblen Schädigung im Sinne einer Nekrose. Als besonders problematisch wird die Austrocknung des Dentins angesehen. Durch Flüssigkeitsentzug und Veränderung der Druckverhältnisse in den Dentinkanälchen können Odontoblastenkerne in die Kanälchen gesaugt werden. Dieser Vorgang wird häufig als Odontoblastenaspiration bezeichnet. Wenn keine ernsthaften Schädigungen erfolgen, ist die resultierende Entzündungsreaktion der Pulpa allerdings weitgehend reversibel (Hellwig et al., 1999).

\subsubsection{Zahnextraktionen}

Die Indikationskataloge in der Literatur folgen keinen starren Regeln. Regelmäßig aufgeführt werden:

- ausgedehnte kariöse Zerstörung des Zahns (keine Erhaltungswürdig- oder Fähigkeit)

- bei Erkrankungen der Pulpa bzw. des apikalen Parodonts, wenn eine Erhaltung mit endodontischen Mitteln (konservativ und chirurgisch) unmöglich ist

- bei weit fortgeschrittenen Parodontopathien

- bei traumatisch geschädigten Zähnen, die nicht erhaltungsfähig sind

- bei Zähnen im Bruchspalt einer Kieferfraktur, wenn sie für den weiteren

funktionellen Ablauf nicht erforderlich sind

- Zähne im Bereich eines malignen Tumors

- Zähne im Bereich einer Epulis bei deren Rezidiv

- Molaren, die eine Kieferhöhleninfektion unterhalten

- bei entsprechender kieferorthopädischen Indikation

- bei entsprechender prothetischer Indikation

(Weber, 1999). 


\subsubsection{Keramikfrakturen}

Grundsätzlich ist Keramik zur Aufnahme von Druckspannungen geeignet, Zugspannungen müssen jedoch vermieden werden. Frakturen bei Keramikkronen können sich dann einstellen, wenn mit der gewählten Indikation die Herstellerempfehlung überschritten wird. Ungeeignete Präparationen, zu dünne Wandstärken und Bearbeitungsfehler können Zugspannungen oder Mikrorisse im Werkstoff verursachen, die Monate später eine Fraktur auslösen können.

Unter dem Begriff „Chipping“ fast man zwiebelförmige Abscherungen (keine Abplatzungen, sondern Defekte innerhalb der Verblendkeramik) zusammen, ohne dass diese bis in die Tiefe des Gerüstes (Keramik, Metall) reichen. Die Ursachen für dieses Verhalten sind bisher nicht sicher bekannt. Vermutet wird u.a. ein unterschiedlicher Schrumpfungskoeffizient während der Abkühlung, welcher zu inneren Spannungen der Verblendkeramik führt (Swain, 2009).

$\mathrm{Da}$ in vivo eingegliederte Restaurationen bestimmungsgemäß vielfältigen mechanischen Belastungen ausgesetzt sind, stellt die Widerstandsfähigkeit des verwendeten Materials gegen Formveränderungen oder Bruch bei Einwirkung äußerer mechanischer Belastung wohl eines der wichtigsten Kriterien für die Beurteilung der Verwendbarkeit von Vollkeramiken dar.

Die vornehmlich beim Kauen, Schlucken oder Zähnepressen ausgelösten Biegebeanspruchungen sind dabei nicht nur die am häufigsten auftretenden, sondern auch die am meisten kritischen Lastfälle. In Abhängigkeit von Richtung, Angriffspunkt und Höhe der dabei einwirkenden Kräfte können innerhalb einer solchermaßen belasteten Restauration, wenngleich eher kurzzeitig, sehr hohe mechanische Spannungen, insbesondere in Form von Zug-, Druck- und Schubspannungen, auftreten. Körber et al. (1984) ermittelten dafür maximale Kaubelastungen von $200 \mathrm{~N}$ im Frontzahnbereich und $300 \mathrm{~N}$ im Seitenzahnbereich mit einer durchschnittlichen Häufigkeit von einer Kraftspitze pro Tag. Würden die in der Restauration aufgebauten mechanischen Spannungen die Widerstandsfähigkeit des jeweils verwendeten Materials übersteigen, so wäre mit einem Versagen der Restauration zu rechnen, was sich bei Keramikkronen in Form von Sprüngen, Abplatzungen oder gar einem vollständigen Bruch der Restauration zeigen kann. Zur Vermeidung eines aus mechanischen Belastungen resultierenden Versagens der Restauration sind sowohl die Widerstandsfähigkeit gegen Formveränderungen, als 
auch die Belastbarkeit der darin verwendeten Materialien in Abhängigkeit von den zu erwartenden mechanischen Spannungen entsprechend hoch zu wählen.

Bei vollkeramischen Restaurationen kann ein Bruch zum einen durch eine einmalige überschwellige Belastung ausgelöst werden. Hierbei überschreitet die einwirkende Kraft, die durch physiologische Kaubewegungen oder aber auch durch parafunktionelle Belastungen hervorgerufen sein kann, die gegenwärtige Gesamtfestigkeit der Restauration (Schwickerath, 1987). Zum anderen kann ein Bruch bei Abnahme der Festigkeit des Materials aber auch durch unterschwellige Beanspruchungen herbeigeführt werden, da beispielsweise thermische, mechanische, abrasive und korrosive Einflüsse zur Verringerung der ursprünglichen mechanischen Belastbarkeit führen können (Schwickerath, 1987).

Verschiedene Untersuchungen belegen, dass im wechselhaften Milieu der Mundhöhle ablaufende chemische Vorgänge und Abrieb Dentalkeramiken erheblich verschleißen und somit eine Minderung von deren Festigkeit zur Folge haben können (Kappert et al., 1989). Die Abnahme der Festigkeit lässt sich dabei unter anderem auch auf das Hydrolyseverhalten zurückführen, das bei Schwickerath und Coca (1987) auch als „statische Ermüdung“ bezeichnet wird. Ausgehend von Mikrofehlern im Gefüge der Keramik, z.B. Poren, Mikrorisse an der Oberfläche oder Einschlüsse im Material, können sich Risse ausbilden und unter wiederholter mechanischer oder thermischer Belastung, unterstützt durch Risskorrosion bei feuchtem Milieu, im Material ausbreiten. Diese Rissbildung ist bei den in der Mundhöhle vorherrschenden niedrigen Temperaturen irreversibel (Schwickerath, 1987). 


\section{$3 \quad$ Material und Methoden}

\subsection{Patientengut}

\subsubsection{Systematische Aktenanalyse}

In einer retrospektiven Querschnittsanalyse werden alle Patienten der Abteilung Prothetik der Universitätsmedizin Göttingen, die im Zeitraum von 1991 bis 1999 mit vollkeramischen In-Ceram-Kronen versorgt wurden, erfasst. Die Datenerhebung erfolgt durch Auswertung der vorliegenden Behandlungsdokumentation und einer klinischen Untersuchung.

\subsubsection{Klinische Nachuntersuchung}

Die Nachuntersuchung erfolgte nach telefonischer Terminvereinbarung bzw. im Rahmen der routinemäßigen Nachkontrolle.

Die Patienten wurden über die Studie aufgeklärt und die klinische Nachuntersuchung erfolgte erst nach der Einwilligungserklärung (Im Anhang: Patientenaufklärungstext und Einwilligungserklärung).

Von Seiten der Ethikkommission bestanden keine ethischen und rechtlichen Bedenken gegen die Durchführung des Forschungsvorhabens (Antragsnummer 2/2/09, siehe Anhang).

Insgesamt wurden im Zeitraum von September 2008 - Juli 2009 bei 60 Patienten 229 Restaurationen nachuntersucht. Bei den Patienten $(n=60)$ handelt es sich zu 51,6\% (n=31) um Frauen und zu 48,4\% (n=29) um Männer.

Für alle Patienten werden bei der Nachuntersuchung folgende Parameter erfasst:

- Geschlecht

- Alter.

Für jede Restauration werden folgende Parameter erfasst:

- Lokalisation

- Art der Zementierung

- Verlust der Restauration 
- Lockerung der Restauration

- Vitalität der Pfeilerzähne

- Sekundärkaries

- Frakturen der Gerüstkeramik

- Frakturen der Verblendkeramik.

Einschlusskriterien:

- alle erforderliche Informationen sind vorhanden bzw. verfügbar.

Ausschlusskriterien:

- erforderliche Informationen sind nicht verfügbar.

\subsection{Untersuchungsablauf}

Für jeden Patienten wurde ein Dokumentationsformular ausgefüllt (siehe Anhang). Die Untersuchung gliederte sich in 2 Abschnitte.

- Datenerfassung mit Hilfe der vorliegenden Behandlungsdokumentation

- Klinische Nachuntersuchung der Restauration/en

Die Datenerfassung mit Hilfe der vorliegenden Behandlungsdokumentation erfasste folgende Dokumentationspunkte:

- Pseudonymisierung

- Geburtsdatum des Patienten

- Datum der Untersuchung

- Fallnummer für die statistische Auswertung

- FDI-Zuordnung des Zahnes

- Art der Restauration

- verwendete Materialien und Eingliederungsdatum

Alle Restaurationen, deren Daten analysiert wurden, wurden durchnummeriert und die Datenblätter (CRFs) pseudonymisiert. Die Pseudonymisierung erfolgt durch Zusammenfassung des jeweils ersten und letzten Buchstabens des Vor- und Nachnamens (z.B. Athanasios Tsigaras: ASTS) sowie der Lokalisation der evaluierten Restauration (z.B. 26) zu einem sechsstelligen Code (Bsp.:ASTS26). 
Allen Restaurationen wurden Nummern zugewiesen: Die Restaurationen wurden in der Reihenfolge ihres Einschlusses nummeriert: INC-001 bis INC-229.

Als Eingliederungsdatum wurde der Tag dokumentiert, an dem die Restauration definitiv zementiert wurde.

Die klinische Nachuntersuchung erbrachte die nachfolgend aufgelisteten Befunde:

- Mundhygienestatus des Patienten

- Restauration in situ/Verlust der Restauration

- Vitalitätsverlust

- Randschlussqualität

- Sekundärkaries/Randverfärbungen

- Approximale Kontakte

- Okklusale Kontakte

- Frakturanfälligkeit.

Der Mundhygienestatus wurde anhand der aktuellen API/SBI-Werte ermittelt. Die Einteilung erfolgte in:

- Sehr gut

- Gut

- Befriedigend

- Unzureichend

- Schlecht.

Die klinische Inspektion zeigte, ob sich die eingegliederte Restauration noch in situ befand oder nicht. Bei einer erfolgten Entfernung der Restauration wurde der Grund für die Neuanfertigung bzw. Entfernung der Aktendokumentation entnommen bzw. durch Befragung des Patienten ermittelt.

Eine Sensibilitätsprüfung mit Hilfe eines mittels $\mathrm{CO}_{2}$-Schnee abgekühlten Schaumstoffpellets erlaubte Aufschlüsse über einen möglichen Vitalitätsverlust des untersuchten Zahnes. Falls ein Röntgenbild vorlag wurde zusätzlich ein radiologischer Befund erhoben. Die Klopfempfindlichkeit eines Zahnes wurde mit Hilfe des Perkussionstestes ermittelt. Mit dem Sondengriff wurde der Zahn in horizontaler und in vertikaler Richtung leicht angeklopft. Ist durch die Perkussion ein Schmerz 
induziert worden, wurde die Klopfempfindlichkeit auf dem Untersuchungsbogen vermerkt.

Die Randschlussqualität der Restaurationen wurde mit einer fabrikneuen zahnärztlichen Sonde geprüft. Mögliche Randunregelmäßigkeiten konnten durch gezieltes Auf- und Abbewegen der Sondenspitze am Kronenrand ertastet werden. Die Einteilung erfolgte in:

- Kein tastbarer Übergang zwischen Restauration und Zahn

- Tastbarer Randspalt an weniger als 50\% des Umfangs der Restauration

- Tastbarer Randspalt an mehr als 50\% des Umfanges der Restauration

- Tastbarer kontinuierlicher Randspalt an der gesamten Restauration.

Die Diagnose Sekundärkaries/Randverfärbung wurde sowohl visuell, als auch instrumentell mit der Sonde gestellt. Die Abstufung erfolgte in:

- Keine Verfärbungen zwischen Restauration und Zahn

- Lokale Verfärbungen zwischen Restauration und Zahn

- Kontinuierliche Verfärbungen zwischen Restauration und Zahn

- Kavitätenbildung mit erweichter Zahnhartsubstanz .

Die Kontaktpunkte wurden mit ungewachster Zahnseide überprüft. Die Einteilung der Approximalkontakte erfolgte in:

- Kontakte optimal gestaltet

- Mesialer Kontaktpunkt etwas zu schwach

- Mesialer Kontaktpunkt etwas zu stark

- Distaler Kontaktpunkt etwas zu schwach

- Distaler Kontaktpunkt etwas zu stark

- Mesialer Kontaktpunkt fehlt

- Mesialer Kontaktpunkt mit Zahnseide nicht gängig

- Distaler Kontaktpunkt fehlt

- Distaler Kontaktpunkt mit Zahnseide nicht gängig.

Die Okklusionskontakte wurden durch Okklusionsfolie überprüft und die Oberflächenrauhigkeit der Okklusionsfläche mit Hilfe einer zahnärztlichen Sonde beurteilt. Die Kontaktpunkte wurden folgendermaßen bewertet:

- Optimale okklusale Abstützung der Restauration 
- Restauration ist geringgradig zu hoch

- Restauration hat etwas zu schwache okklusale Kontaktpunkte

- Lokale Schlifffacetten an der Restauration

- Lokale Schlifffacetten am Antagonisten

- Starke Abrasionszeichen an der Restauration

- Starke Abrasionszeichen an den Antagonisten

- Restauration verursacht eine klinisch manifeste Funktionsstörung.

Eine Riss-/Frakturbildung wurde in folgende Kategorien eingeteilt:

- Kein Defekt

- Riss oder Sprung in der Verblendkeramik

- Fraktur in der Verblendkeramik

- Riss oder Sprung im Gerüst

- Fraktur im Gerüst.

\subsection{Statistische Analyse}

Jede Restauration wurde als statistische Einheit definiert. Ferner wurde eine Clusteranalyse in zwei Teilmengen der Stichproben vorgenommen (Einzelkrone im Frontzahnbereich, Einzelkrone im Seitenzahnbereich).

Die statistische Auswertung erfolgt mit einem Computerprogramm (Systat ${ }^{\mathrm{TM}}$ for Windows Version 10, Systat Inc. Evanston, USA).

Die zeitabhängige Überlebenswahrscheinlichkeit wurde nach der Kaplan-MeierMethode (Kaplan und Meier, 1958) berechnet. Diese Methode wird bei nicht normal verteilten Daten zur Berechnung der Überlebenszeiten verwendet, und ist sowohl bei kleinen, als auch bei mittleren und großen Fallzahlen anwendbar. Ein Vorteil besteht darin, dass bei der Überlebenszeitanalyse sowohl unzensierte wie auch zensierte Daten verwendet werden können.

Die Auswertung erfolgte unter Bewertung zweier wesentlicher Kriterien:

- „Überleben“: Ist die Restauration noch in Funktion (in-situ-Kriterium)?

- „Erfolg“: Musste während der Liegezeit der Restauration klinisch interveniert werden (Ereignisfreiheit)? 


\section{$4 \quad$ Ergebnisse}

4.1 Charakterisierung des Untersuchungsgutes

\subsubsection{Das Patientengut}

Nachfolgend werden die unterschiedlichen Auswertungen hinsichtlich

Geschlechtsverteilung und Anzahl der Restaurationen patientenbezogen dargestellt.

\subsubsection{Geschlechtsverteilung}

Bei den nachuntersuchten Patienten $(n=60)$ handelt es sich $\mathrm{zu} 51,6 \%(\mathrm{n}=31)$ um Frauen und zu 48,4\% (n=29) um Männer.

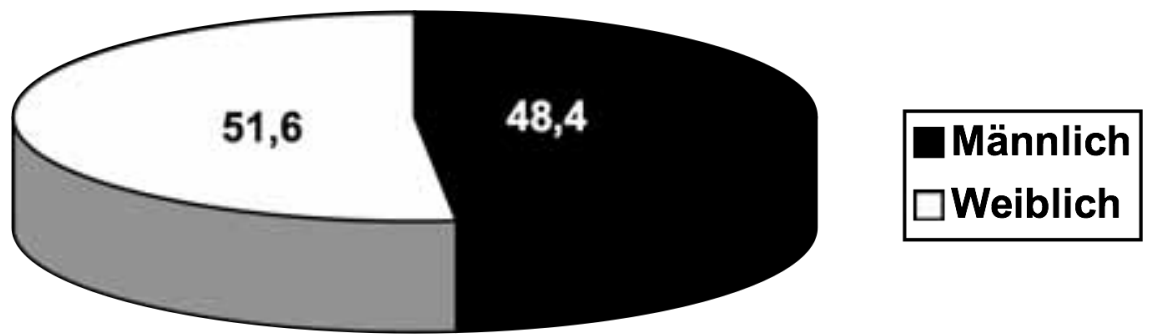

Diagramm 1: Geschlechtsverteilung der 60 untersuchten Patienten in Prozent. 


\subsubsection{Mundhygienestatus}

Gut $72 \%$ der Patienten wiesen eine gute bzw. sehr gute Mundhygiene auf. Lediglich $3 \%$ zeigten einen unzureichenden Status.

\section{Mundhygienestatus $(n=60)$}

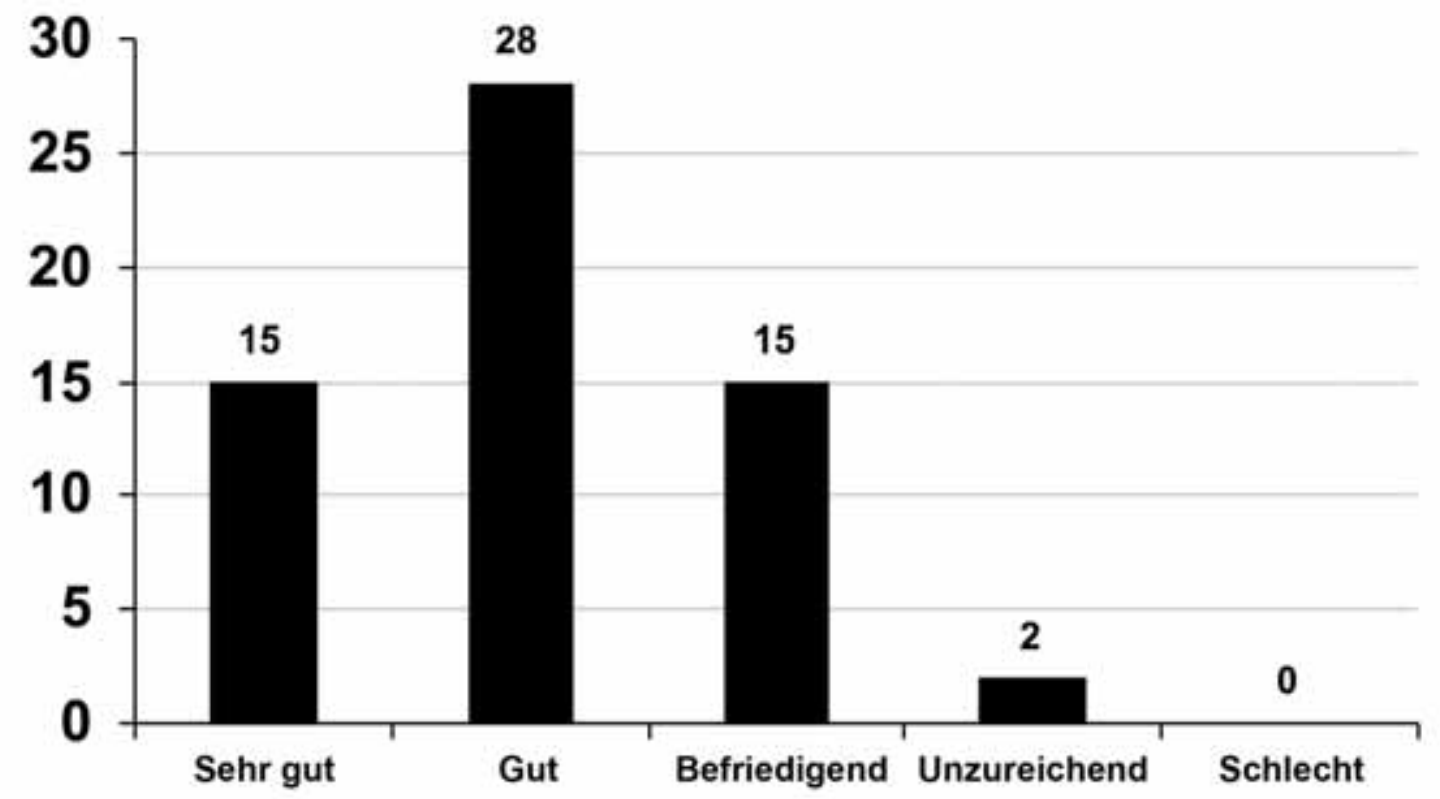

Diagramm 2: Überblick über den Mundhygienestatus der Patienten zum Zeitpunkt der klinischen Nachuntersuchung. 
4.1.1.3 Häufigkeitsverteilung der Restaurationen pro Patient

Diagramm 2 gibt Auskunft über die Anzahl der Restaurationen, die pro Patient eingegliedert wurden.

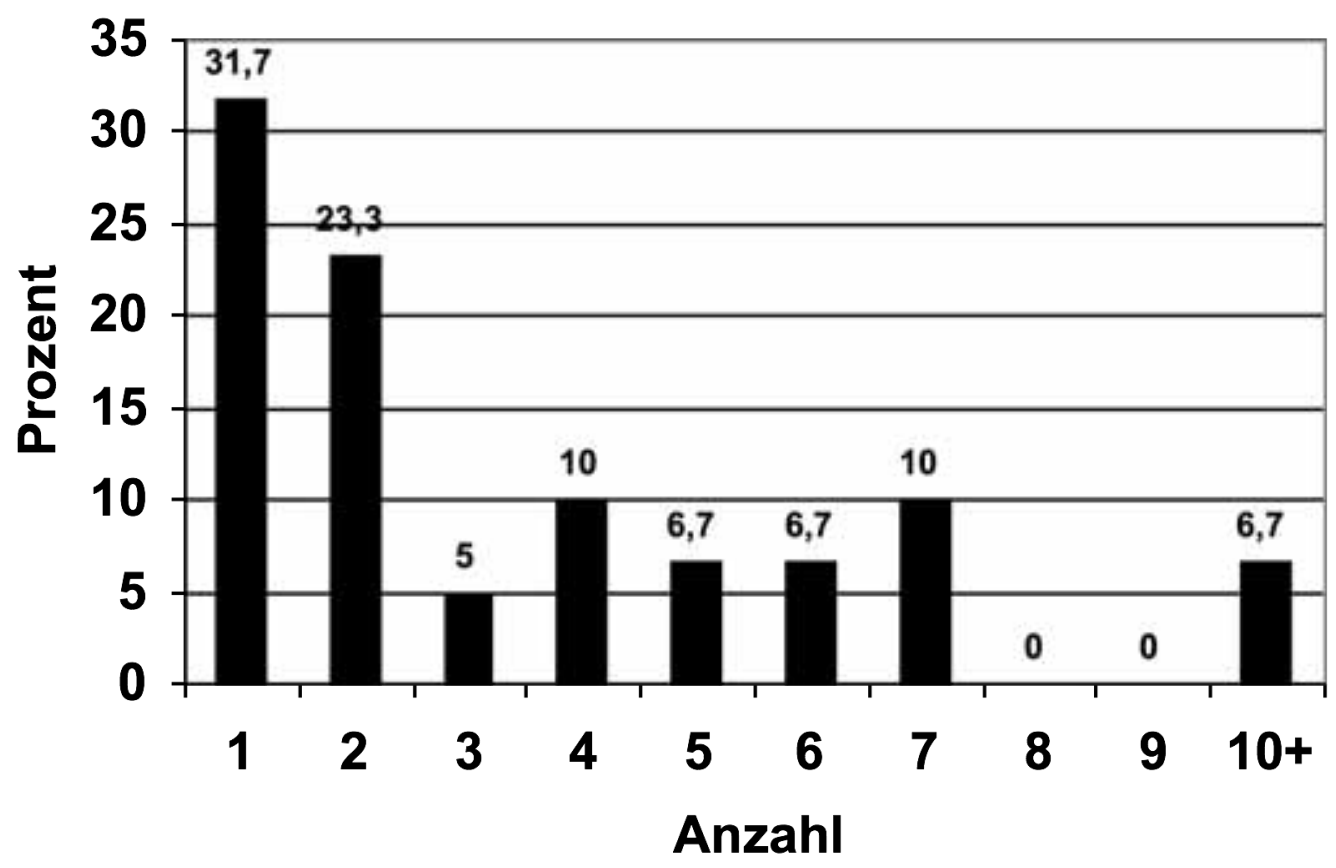

Diagramm 3: Häufigkeitsverteilung der Restaurationen pro Patient. 


\subsubsection{Die Restaurationen}

Die Ergebnisse hinsichtlich der Verteilung auf die verschiedenen Zähne und der Art der Zementierung werden in diesem Kapitel herausgestellt.

\subsubsection{Verteilung der Restaurationen bezogen auf die einzelnen Zähne (FDI)}

Die Verteilung der Restaurationen bezüglich ihrer Lokalisation in Ober- und Unterkiefer wird aus dem Diagramm 3 deutlich.

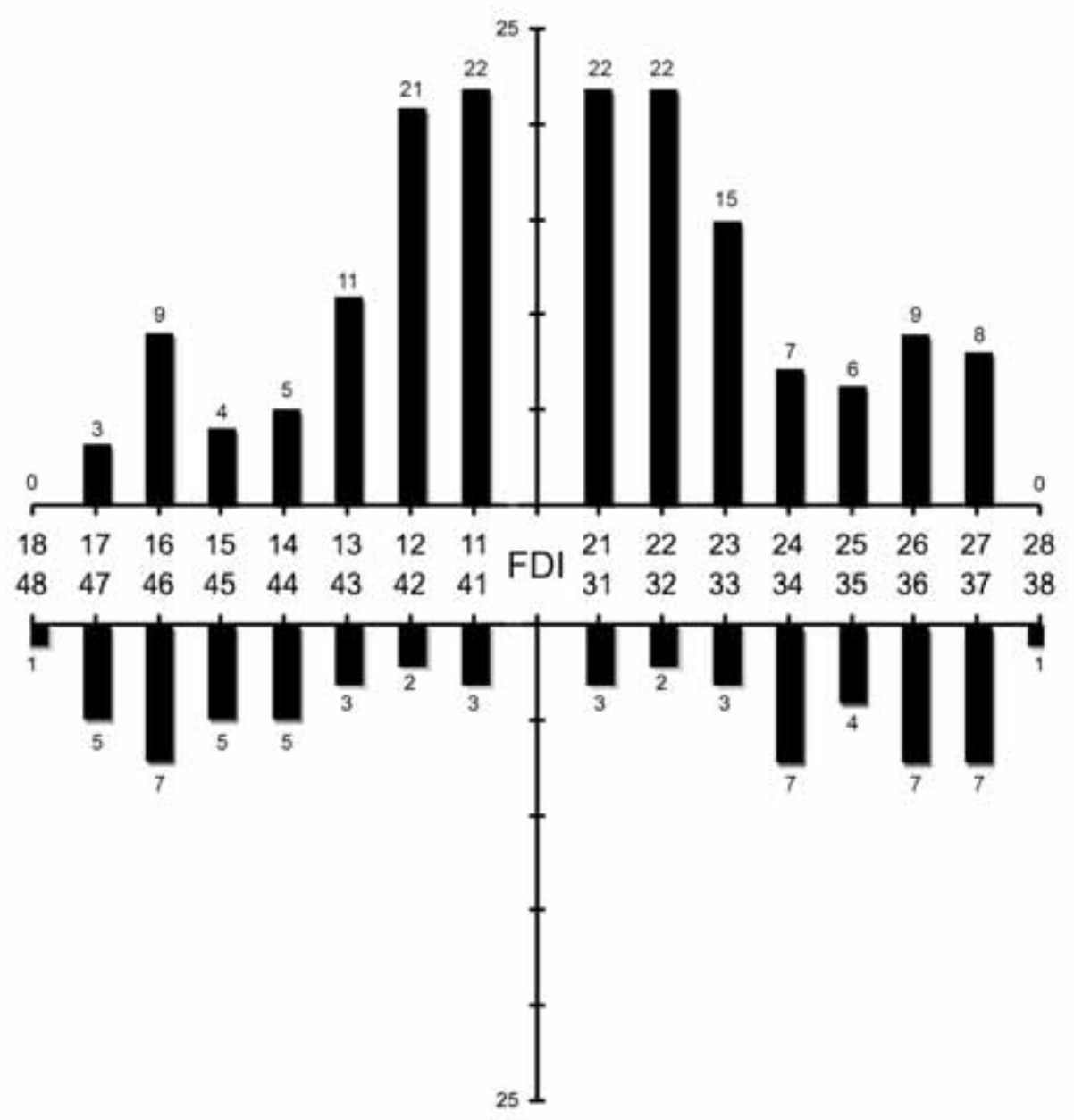

Diagramm 4: Häufigkeitsverteilung der Restaurationen bezogen auf die einzelnen Zähne (FDI). 


\subsubsection{Art der Zementierung}

Die Datenerfassung mit Hilfe der vorhandenen Patientenakten erfasste folgende verwendete Materialien zur definitiven Eingliederung der Restaurationen: Ketac Cem (3M Espe, Neuss), Dyract Cem (Dentsply, Konstanz), Compolute (3M Espe, Neuss), Harvard (Richter \& Hoffmann, Hoppegarten), Fleck`s (Mizzy Inc., Cherry Hill, USA), Panavia 21 (Kuraray Dental, Frankfurt/Main).

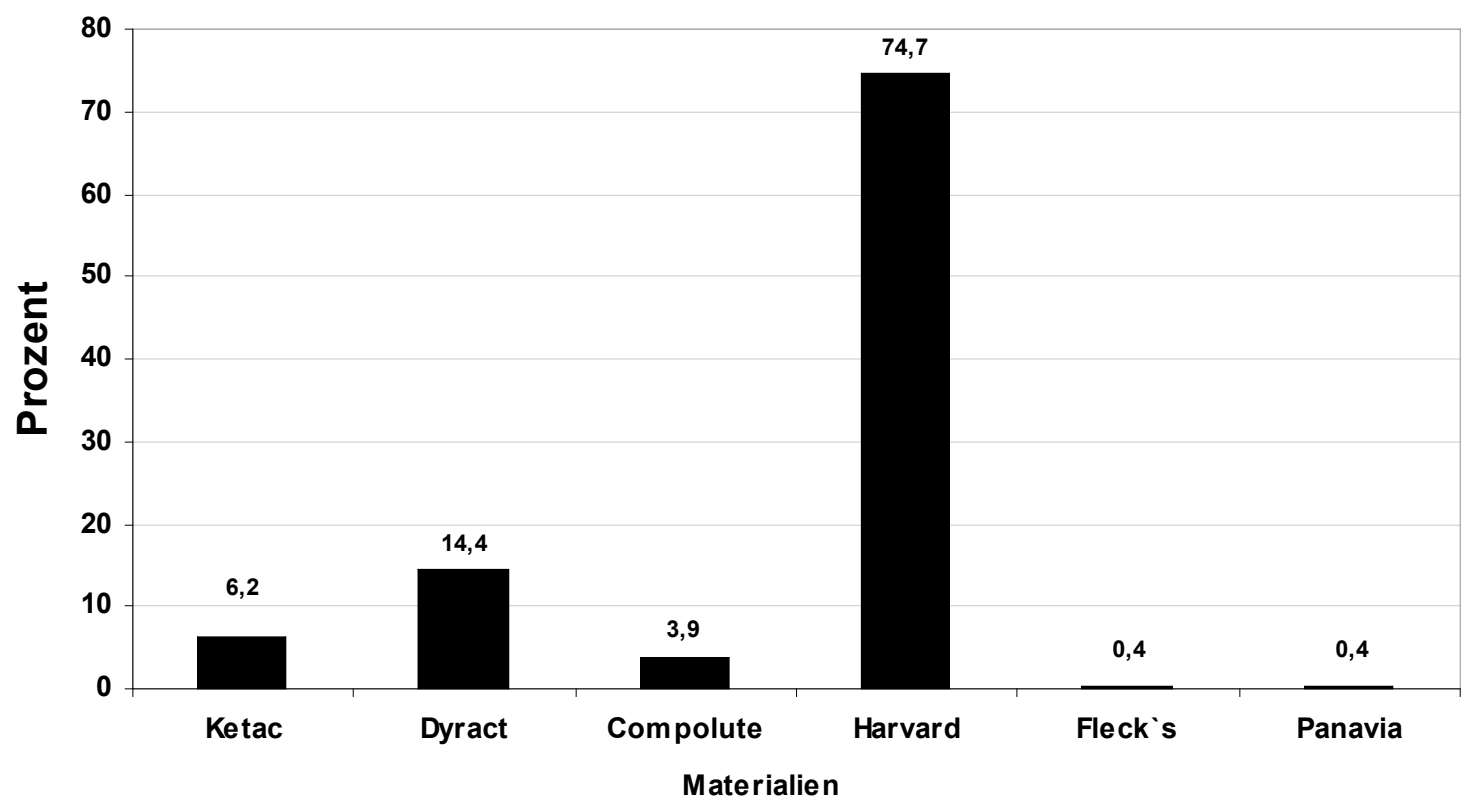

Diagramm 5: Verwendete Befestigungsmaterialien.

\subsubsection{Endodontische Vorbehandlung der Pfeilerzähne}

33 von 229 Restaurationen (14,4\%) wurden auf devitalen endodontisch vorbehandelten Pfeilerzähnen hergestellt. Vital zum Zeitpunkt der definitiven Eingliederung waren demnach 196 Pfeilerzähne (85,6\%). Bei 6 dieser vitalen Pfeilerzähne $(3,1 \%)$ trat ein Vitalitätsverlust nach prothetischer Versorgung ein. 
4.1.2.4 Liegezeiten der Restaurationen

Die in dieser Studie erfassten Restaurationen hatten eine mittlere Liegezeit von 12,8 Jahren bei einer Standardabweichung von 3,6 Jahren bei einem maximalen Wert von 17,7 Jahren.

4.2 Ergebnisse der Nachuntersuchung

\subsubsection{5-Jahres-Ergebnisse}

\subsubsection{Charakteristika der Restaurationen}

$82 \%$ aller Restaurationen zeigte eine einwandfreie Randschlussqualität. Bei 9,6\% der Kronen war an weniger als 50\% des Umfanges des Zahnes ein Randspalt tastbar, bei $8,7 \%$ war bei mehr als $50 \%$ der zirkulären Präparationsgrenze ein Randspalt sondierbar.

\section{Randschlussqualität}

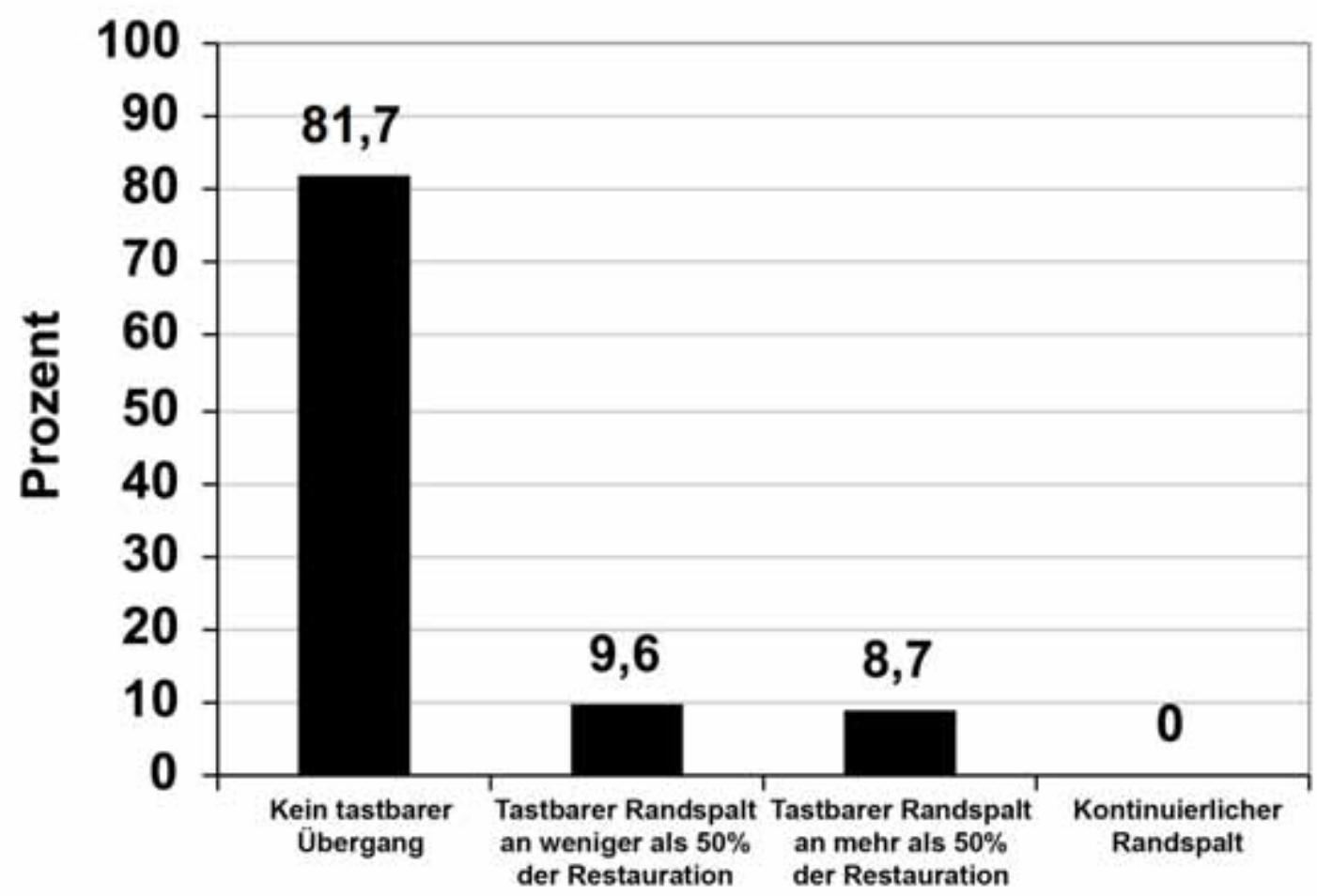

Diagramm 6: Randschlussqualität der Restaurationen nach 12,8 Jahren mittlerer klinischer Verweildauer. 
Lokale Verfärbungen am Kronenrand lagen bei 19,2\% der Restaurationen vor wohingegen 2,2\% kontinuierliche Verfärbungen aufwiesen. Bei 6,1\% war es im marginalen Bereich zu Kavitätenbildungen gekommen. Bei insgesamt 72,5\% ergaben sich für diesen Parameter keine Befunde.

\section{Sekundärkaries}

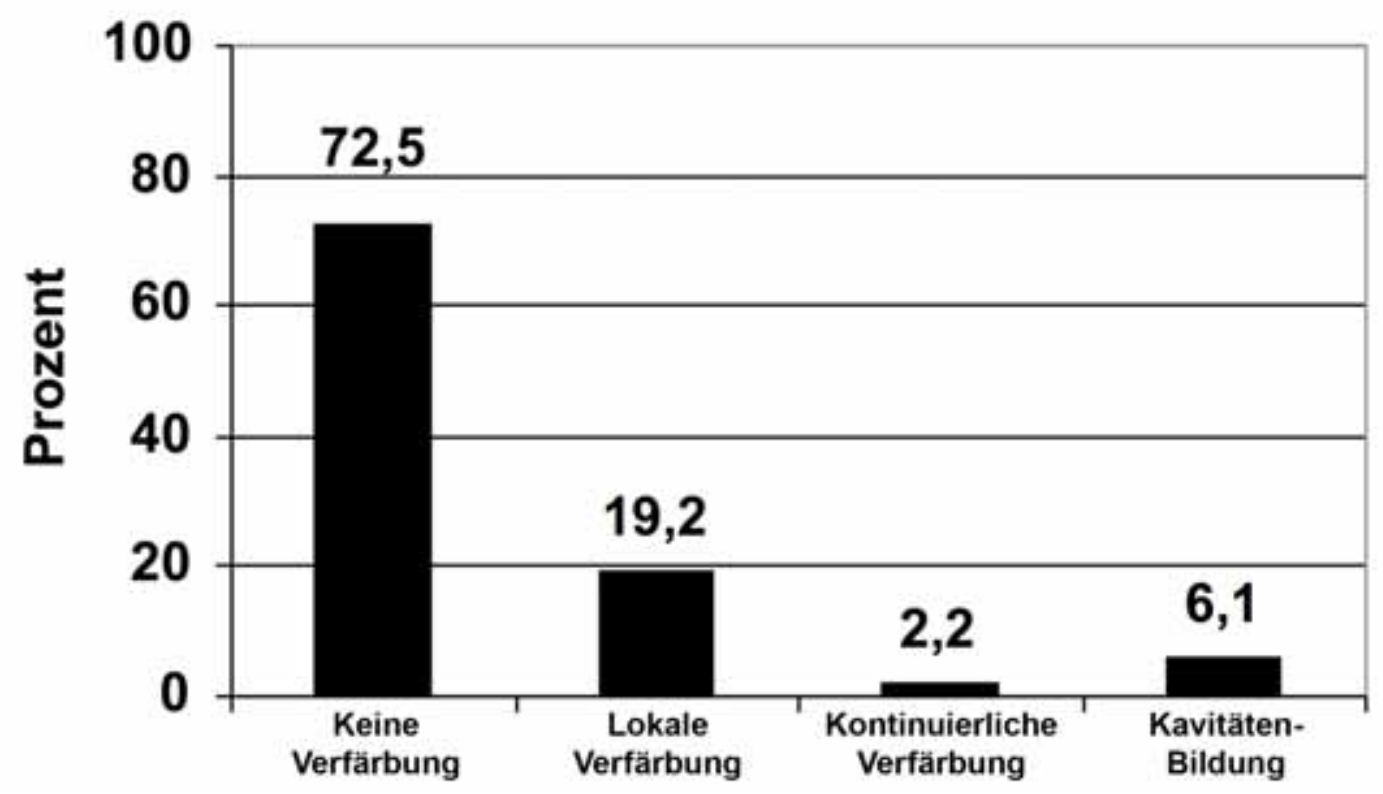

Diagramm 7: Beurteilung des marginalen Saumes der Restaurationen nach 12,8 Jahren mittlerer klinischer Verweildauer.

Die approximalen Kontakte waren bei fast allen Restaurationen als fehlerfrei zu beurteilen (98,7\%). Bei 0,4\% war der distale Kontaktpunkt zu schwach ausgeprägt, bei $0,9 \%$ fehlte dieser vollständig.

Im Bereich der okklusalen Kontaktpunkte waren keine Auffälligkeiten zu verzeichnen. Anhand der fotographischen Dokumentation lässt sich darstellen, dass Frakturen der Keramiken im Seitenzahngebiet vorwiegend im Bereich der zentrischen (tragenden) Höcker aufgetreten sind (Daten nicht im CRF).

\subsubsection{2 Überlebenswahrscheinlichkeit (in-situ-Kriterium)}

Insgesamt wurden 41 Totalverluste von 229 Restaurationen registriert (Tab. 1). Die resultierende Überlebenswahrscheinlichkeit (in-situ-Kriterium) für den 15-JahresZeitraum beträgt 77,3\% (nach Kaplan-Meier) (Diagramm 8). Technische Gründe 
lagen bei 12 der 41 Verluste zu Grunde, wobei großflächige Frakturen der Verblendkeramik oder Frakturen der Gerüste in dieser Kategorie gewertet wurden. Die anderen 19 Fälle entfielen auf biologische Versagensgründe wie Sekundärkaries oder endodontische und parodontologische Misserfolge. Weiterhin mussten 10 Restaurationen im Seitenzahnbereich aufgrund einer Änderung des prothetischen Behandlungsplanes entfernt werden.

Im Frontzahnbereich gingen 17 von 129 Restaurationen verloren (Tab. 1). Dies entspricht für einen 15-Jahreszeitraum einer Überlebensrate nach Kaplan-Meier von 85,9\% (Diagramm 9). Hiervon entfielen 6 Fälle auf technische und 11 Fälle auf biologische Versagensgründe.

Im Seitenzahnbereich wurde eine Überlebenswahrscheinlichkeit (Kaplan-Meier) für In-Ceram-Einzelzahnkronen von 64,6\% für eine klinische Verweildauer von 15 Jahren ermittelt (Diagramm 9). Dies entspricht 24 Verlusten von 100 Seitenzahnrestaurationen, wovon 6 Fälle auf biologische und 8 Fälle auf technische Gründe zurückzuführen waren (Tab. 1).

Ein signifikanter Unterschied zwischen den Überlebensraten von In-CeramEinzelzahnkronen im Front-, relativ zum Seitenzahnbereich, konnte mit Hilfe des LogRang-Testes (Cox-Mantel) dargestellt werden $(\mathrm{p}=0,0239)$.

\subsubsection{Erfolgswahrscheinlichkeit (ereignisfreie Restaurationen)}

Aufgrund von Komplikationen musste bei 28 Restaurationen klinisch interveniert werden, wobei diese allerdings in den meisten Fällen noch in Funktion sind (Tab. 2). Lediglich 4 Fälle vorerst minderschwerer Komplikationen wandelten sich im Verlauf der Liegedauer in Totalverluste.

Die häufigste Komplikation ( $\mathrm{n}=11$ ) trat in Form von „Chipping“ der Verblendkeramik auf, gefolgt von Sekundärkaries $(n=10)$. Die Frakturränder- und Flächen der Verblendungsfrakturen wurden geglättet und marginale kariöse Kavitationen mit Kompositfüllungen versiegelt. Endodontische Behandlungen nach eingetretenem Vitalitätsverlust wurden in 4 Fällen notwendig, 3 Fälle von Retentionsverlusten traten auf.

Die Erfolgsrate wurde entsprechend nach Kaplan-Meier für eine klinische Verweildauer von 15 Jahren mit 68,7\% ermittelt (Diagramm 10). 
Im Frontzahnbereich musste bei 13 Fällen (von insgesamt 129 Restaurationen) klinisch nachbehandelt werden, wobei in 6 Fällen nach „Chipping“ poliert und in 4 Fällen nach Vitalitätsverlust endodontisch behandelt werden musste. In einem Fall wurde eine Krone rezementiert und weiteren 2 Fällen kariöse Läsionen adhäsiv versiegelt (Tab. 2). Entsprechend wurde nach Kaplan-Meier eine Erfolgswahrscheinlichkeit von 74,5\% nach 15 Jahren klinischer Verweildauer ermittelt (Diagramm 11).

Im Seitenzahnbereich erfolgte bei 15 von 100 Fällen eine klinische Intervention nach Insertion (8 Versiegelungen nach marginaler Sekundärkaries, 5 Polituren nach „Chipping“, 2 Rezementierungen nach Retentionsverlust; Tab. 2). Dies entspricht einer Erfolgswahrscheinlichkeit (nach Kaplan-Meier für 15 Jahre klinischer Verweildauer) von 54,2\% (Diagramm 11).

Auch im Vergleich der Erfolgswahrscheinlichkeiten für den Front- und für den Seitenzahnbereich ergab sich unter Anwendung des Log-Rang-Tests (Cox-Mantel) ein signifikanter Unterschied ( $\mathrm{p}=0,004)$ im 15-Jahres-Zeitraum.

\subsubsection{0-Jahres-Ergebnisse}

Für 10 Jahre klinische Verweildauer zeigten In-Ceram-Einzelzahnkronen insgesamt eine Überlebenswahrscheinlichkeit (in-situ-Kriterium, nach Kaplan-Meier) von 88,9\%; im Frontzahnbereich betrug sie 89\% und im Seitenzahnbereich 88,8\%.

Die Erfolgswahrscheinlichkeit (ereignisfreie Restaurationen, nach Kaplan-Meier) lag bei 84,1\%. Isoliert für den Frontzahnbereich ergab sich eine Quote von 83,8\% und für den Seitenzahnbereich lag sie bei 84,6\%. 


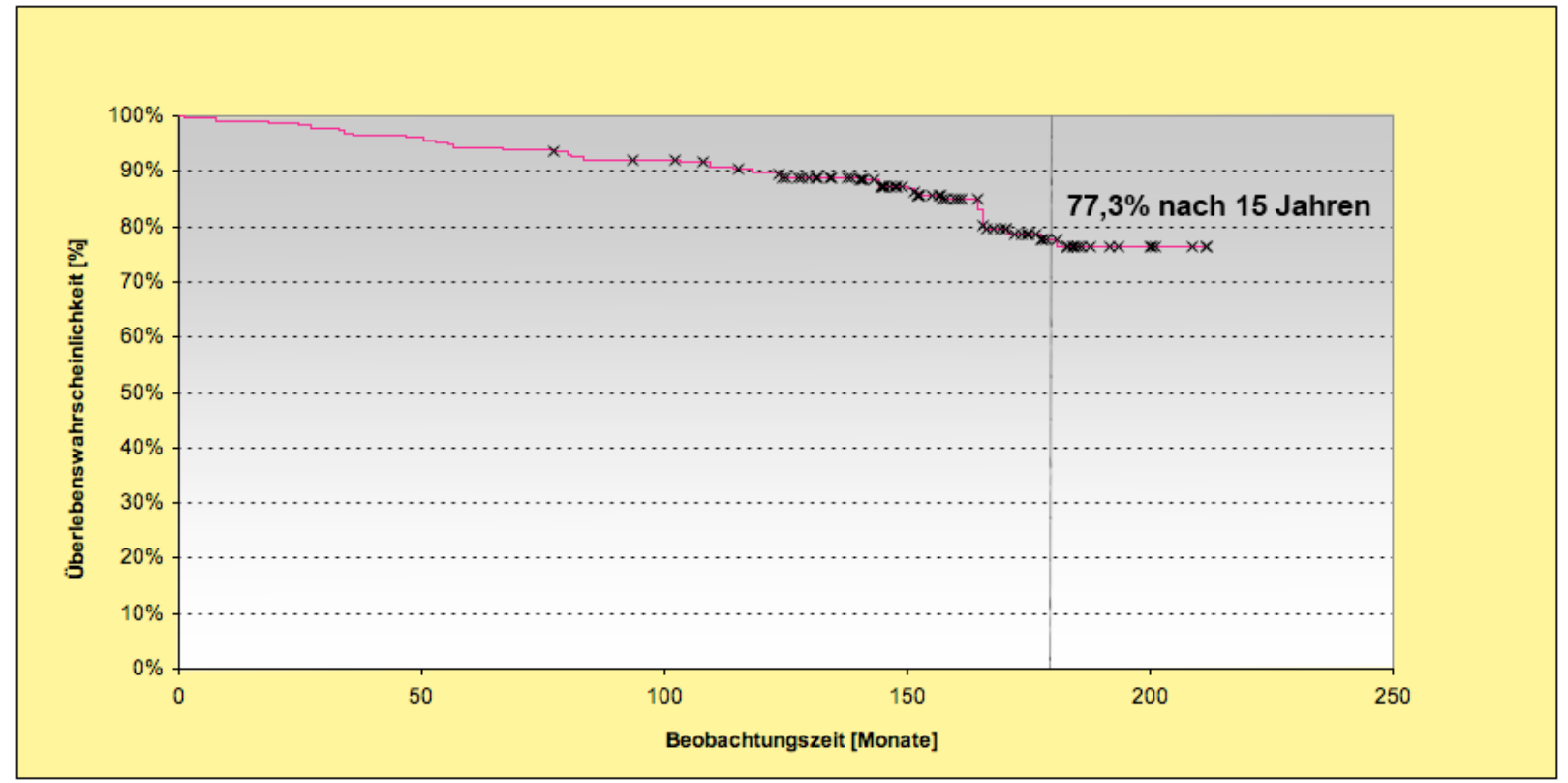

Diagramm 8: Darstellung der klinischen Überlebenswahrscheinlichkeit (in-situ-Kriterium) nach Kaplan-Meier für In-Ceram-Einzelzahnkronen (41 Verluste von insgesamt 229 Restaurationen). 


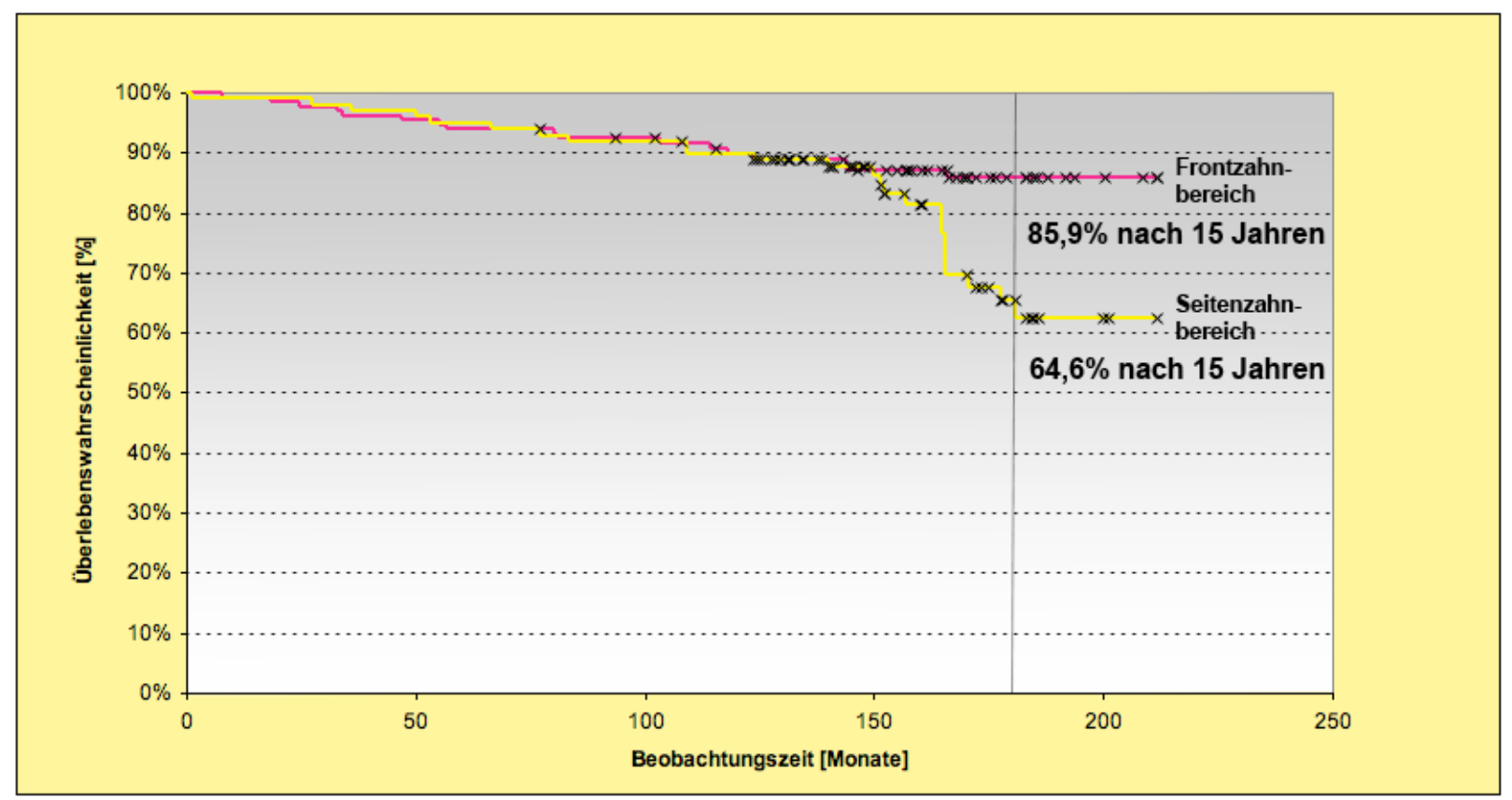

Diagramm 9: Darstellung der klinischen Überlebenswahrscheinlichkeit nach Kaplan-Meier für In-Ceram-Einzelzahnkronen, aufgeschlüsselt nach ihrer Lokalisation im Mund (17/129 Verluste im Frontzahnbereich; 24/100 Verluste im Seitenzahnbereich). 


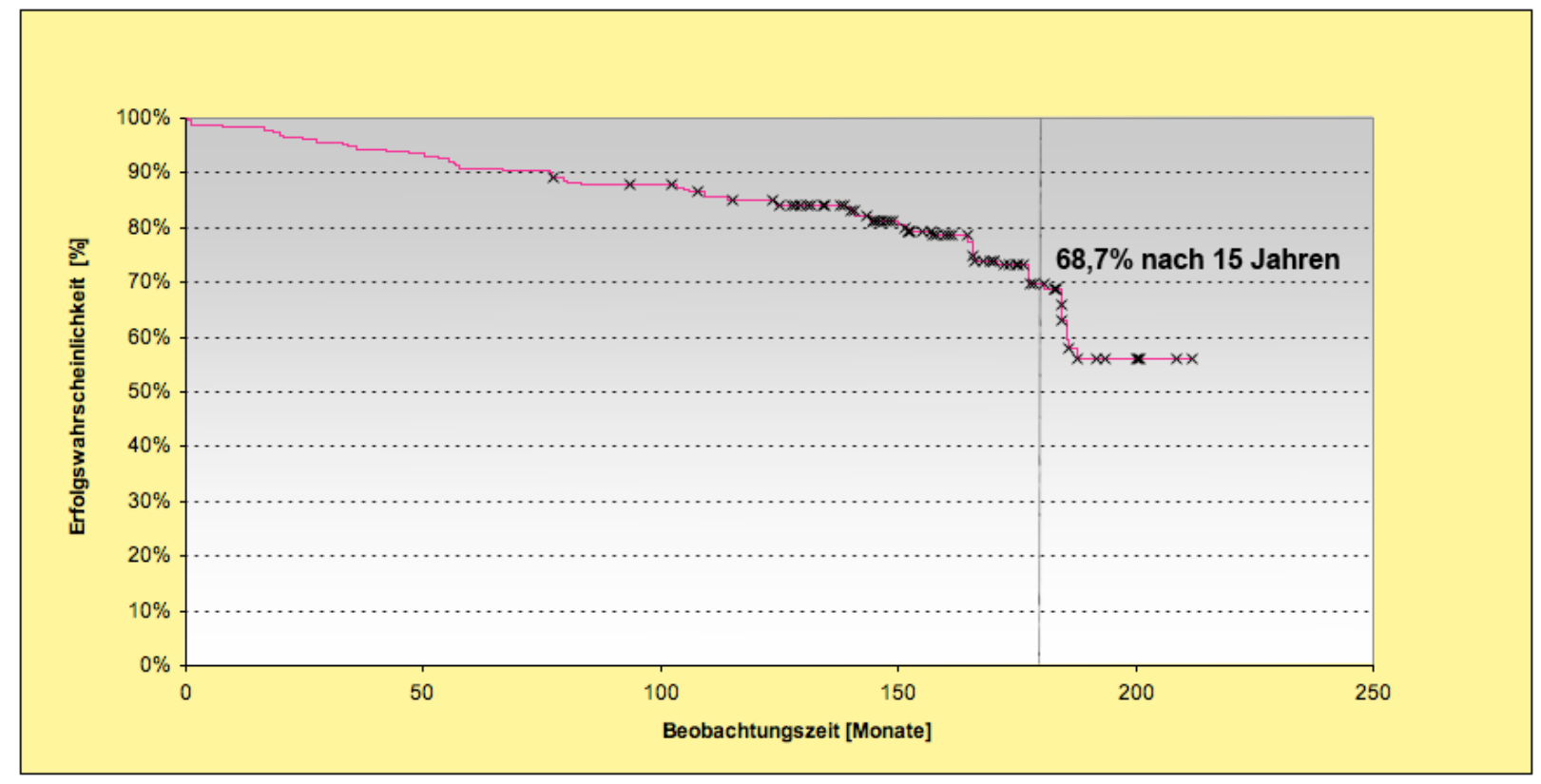

Diagramm 10: Darstellung der klinischen Erfolgswahrscheinlichkeit (Ereignisfreiheit) nach Kaplan-Meier für In-Ceram-Einzelzahnkronen (164 von insgesamt 229 Restaurationen). 


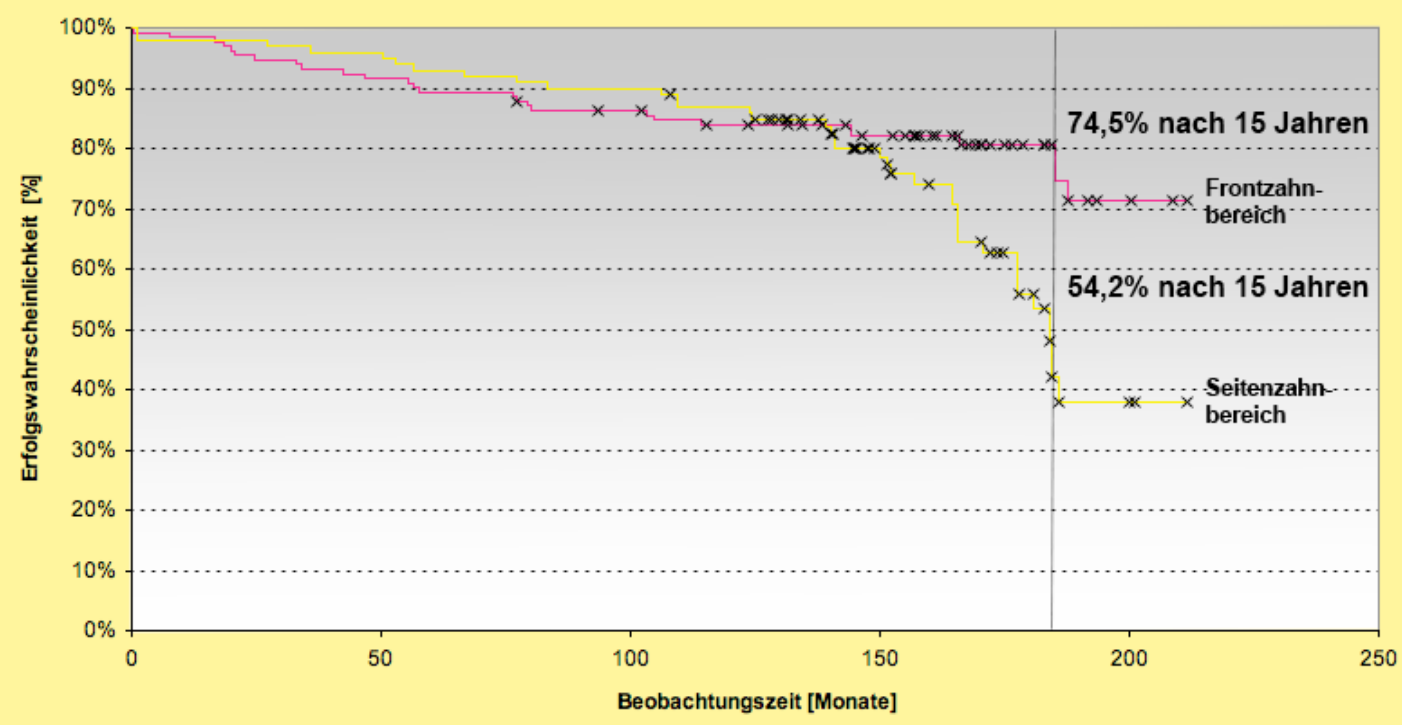

Diagramm 11: Darstellung der klinischen Erfolgswahrscheinlichkeit nach Kaplan-Meier für InCeram-Einzelzahnkronen, aufgeschlüsselt nach ihrer Lokalisation im Mund (102/129 erfolgreiche Restaurationen im Frontzahnbereich; 62/100 im Seitenzahnbereich). 


\begin{tabular}{|c|c|c|c|}
\hline Art des Verlustes & $\begin{array}{c}\text { Frontzahngebiet } \\
(\mathbf{n = 1 2 9})\end{array}$ & $\begin{array}{c}\text { Seitenzahngebiet } \\
(\mathbf{n = 1 0 0 )}\end{array}$ & $\begin{array}{c}\text { Total } \\
(\mathbf{n}=\mathbf{2 2 9})\end{array}$ \\
\hline $\begin{array}{c}\text { Technisch } \\
\text { (Keramik-Frakturen) }\end{array}$ & 6 & 6 & 12 \\
\hline $\begin{array}{c}\text { Biologisch } \\
\text { (Kariologisch, } \\
\text { Endodontisch, } \\
\text { Parodontologisch) }\end{array}$ & 11 & 8 & 19 \\
\hline $\begin{array}{c}\text { Änderung des } \\
\text { Behandlungsplanes }\end{array}$ & 0 & 10 & 10 \\
\hline Total & $\mathbf{1 7}$ & $\mathbf{2 4}$ & $\mathbf{4 1}$ \\
\hline
\end{tabular}

Tabelle 1: Darstellung der aufgetretenen Verluste, aufgeschlüsselt nach ihrer Lokalisation im Mund.

(Anm.: 4 Restaurationen wurden im Verlauf von einer Komplikation zu einem Totalverlust, 3 anteriore und 1 posteriore Restauration).

\begin{tabular}{|c|c|c|c|}
\hline Art der Komplikation & $\begin{array}{c}\text { Frontzahngebiet } \\
(\mathbf{n = 1 2 9})\end{array}$ & $\begin{array}{c}\text { Seitenzahngebiet } \\
(\mathbf{n}=\mathbf{1 0 0})\end{array}$ & $\begin{array}{c}\text { Total } \\
(\mathbf{n = 2 2 9})\end{array}$ \\
\hline Retentionsverluste & 1 & 2 & 3 \\
\hline $\begin{array}{c}\text { Geringgradiges } \\
\text { Chipping }\end{array}$ & 6 & 5 & 4 \\
\hline $\begin{array}{c}\text { Endodontische } \\
\text { Behandlung } \\
\text { Karies }\end{array}$ & 4 & 0 & 10 \\
\hline Total & 2 & 8 & $\mathbf{2 8}$ \\
\hline
\end{tabular}

Tabelle 2: Darstellung der aufgetretenen Komplikationen, aufgeschlüsselt nach ihrer Lokalisation im Mund.

(Anm.: 4 Restaurationen wurden im Verlauf von einer Komplikation zu einem Totalverlust, 3 anteriore und 1 posteriore Restauration). 


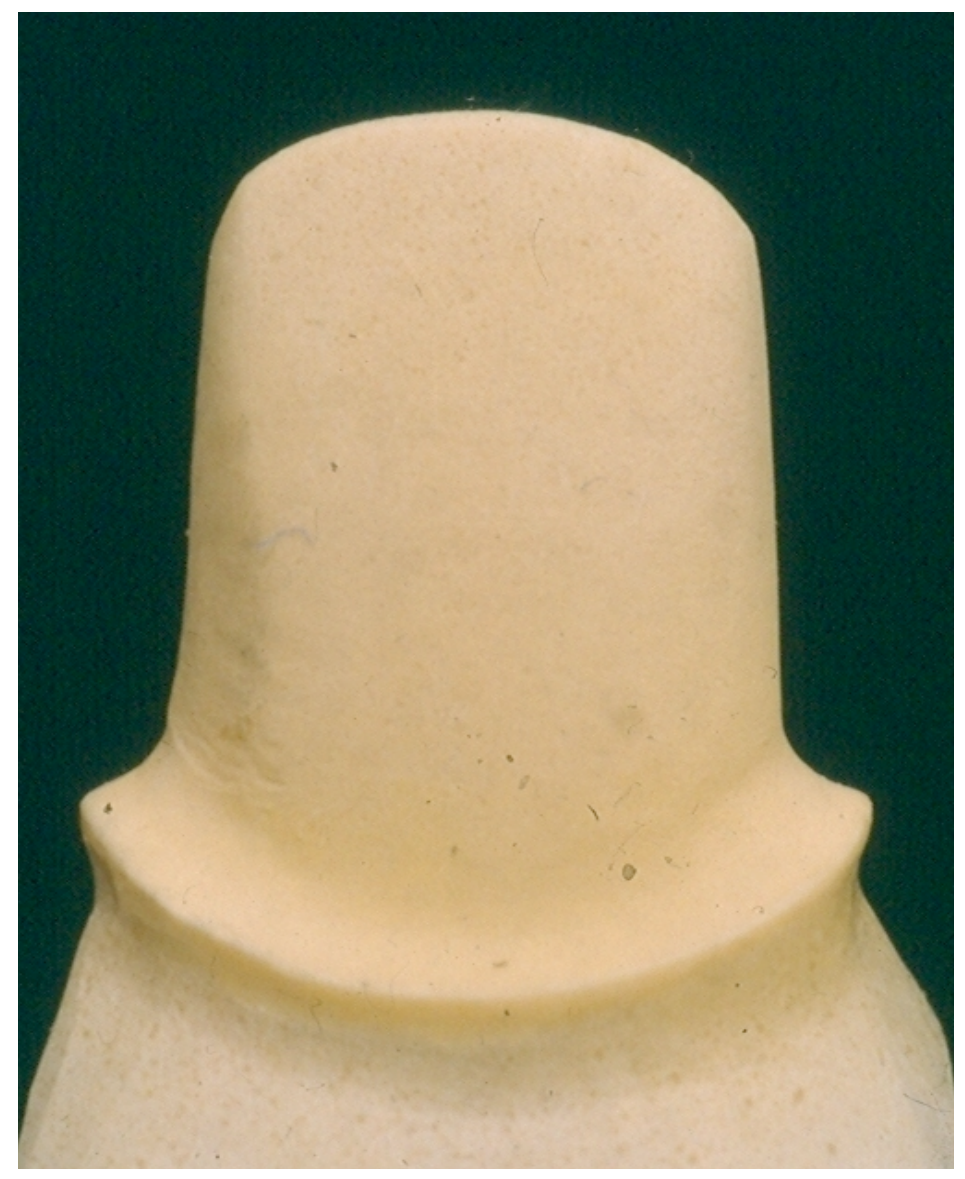

Abbildung 2: Idealisierte Präparation für eine vollkeramische Restauration mit ausgeprägter Hohlkehle (Schnitttiefe ca. 1,5 mm), $2 \times 3^{\circ}$ Konuswinkel und einer Stumpfhöhe von mehr als $4 \mathrm{~mm}$. Eine mechanische Abstützung an der Präparationsgrenze marginal ist notwendig. Voraussetzung ist ein ausreichendes Volumen an Zahnhartsubstanz. 


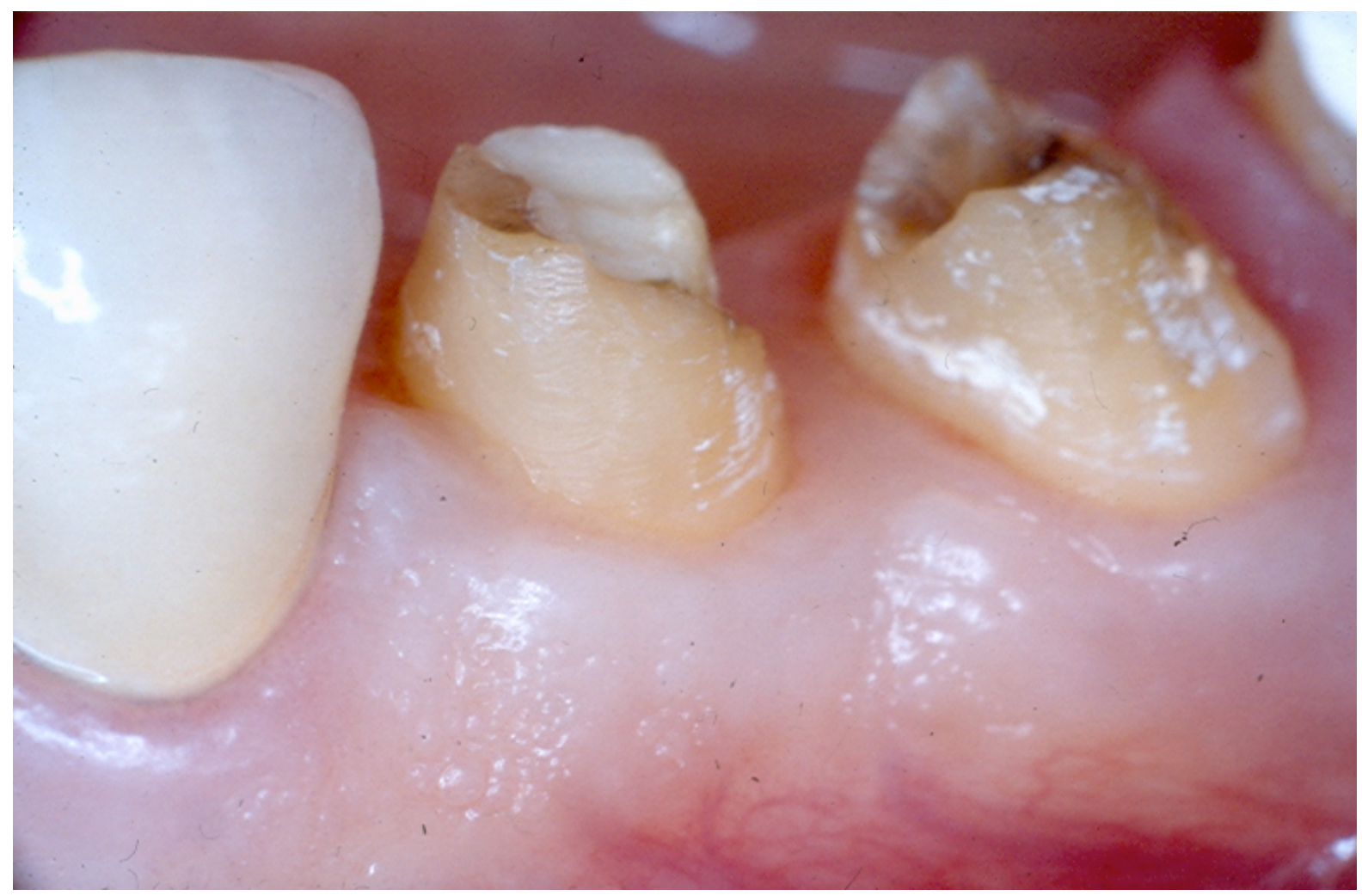

Abbildung 3: Vor allem bei Wiederholungsüberkronungen ist eine Versorgung mit dem InCeram-System aufgrund des reduzierten Hartsubstanzangebotes vergleichsweise oft nicht möglich. Hierdurch wird die Anwendungsbreite für vollkeramische Kronen reduziert.

Die Abbildung zeigt ein Beispiel für die Prämolaren 34 und 35 mit Zustand nach Entfernung insuffizienter metall-keramischer Restaurationen. 


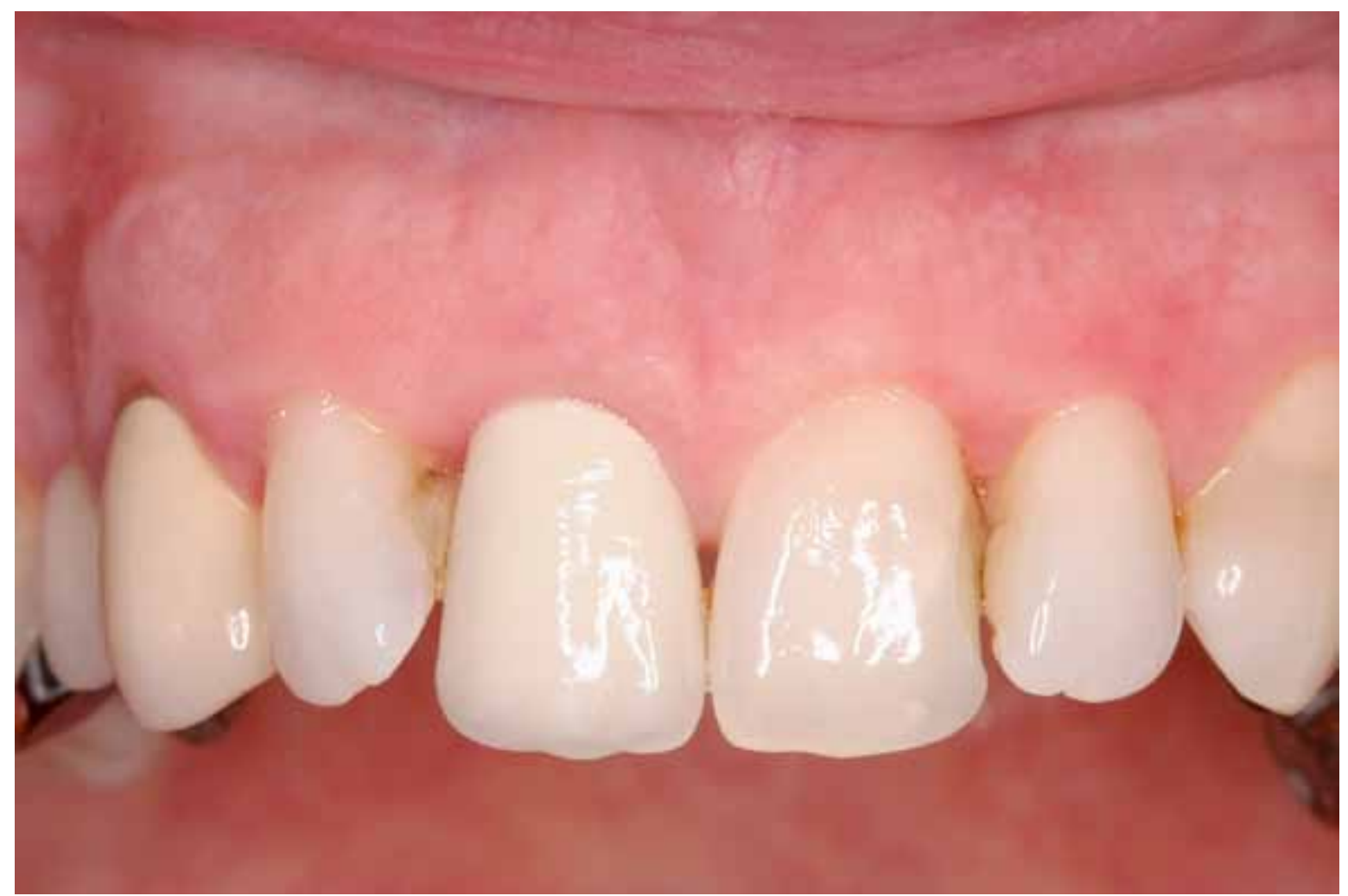

Abbildung 4: Ästhetisch und medizinisch suffiziente In-Ceram-Versorgung des Zahnes 11 bei einem 49-jährigen Patienten nach 16 Jahren klinischer Verweildauer. Auch die gingivalen Verhältnisse erscheinen reizlos und gesund. 


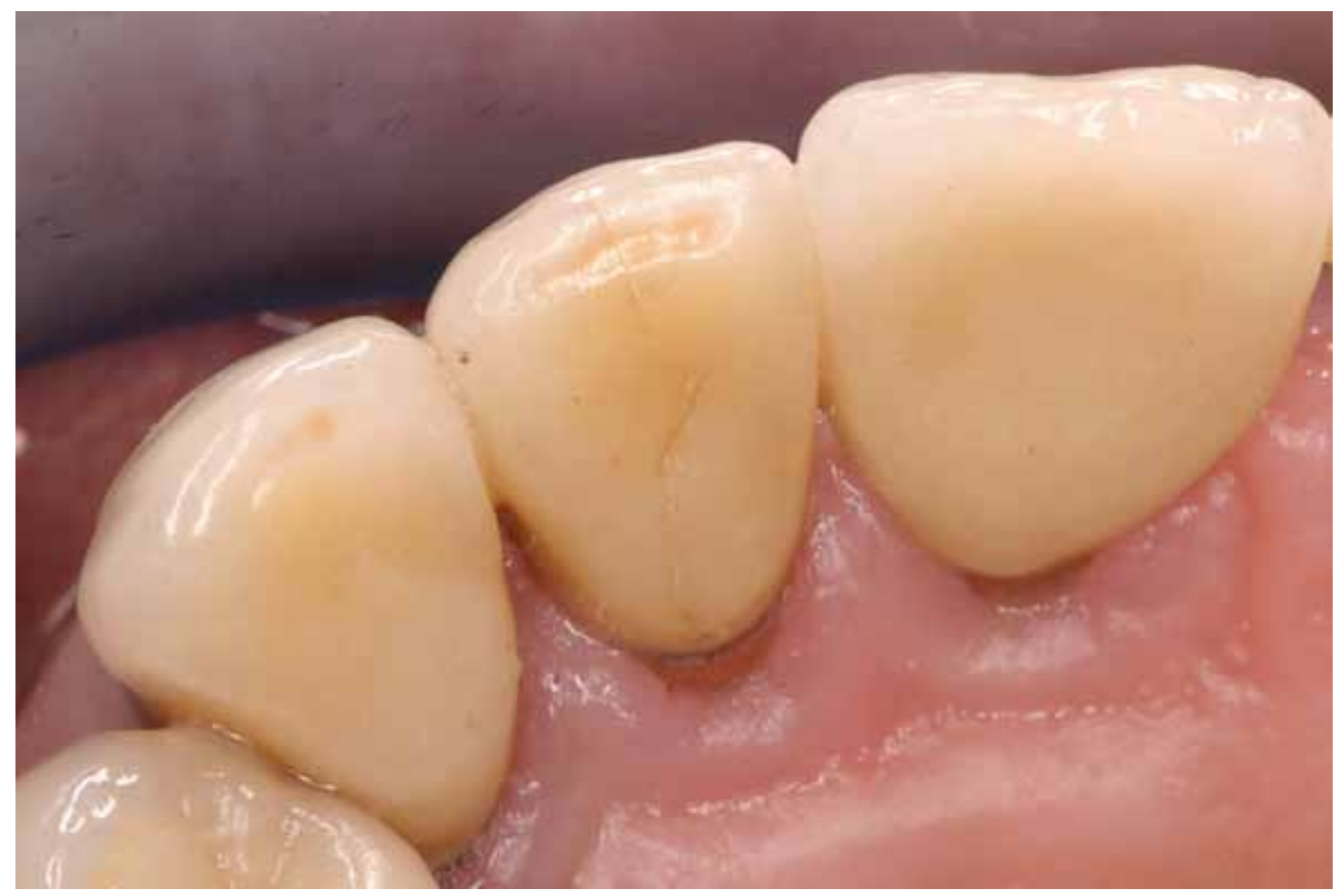

Abbildung 5: In-Ceram-Kronen auf den Zähnen 13-11 bei einem 74-jährigen Patienten nach 16 Jahren klinischer Verweildauer.

Die Krone auf Zahn 12 zeigt einen palatinalen Sprung in der Verblendkeramik und eine Verfärbung am geringfügig überkonturierten Rand palatinal. 


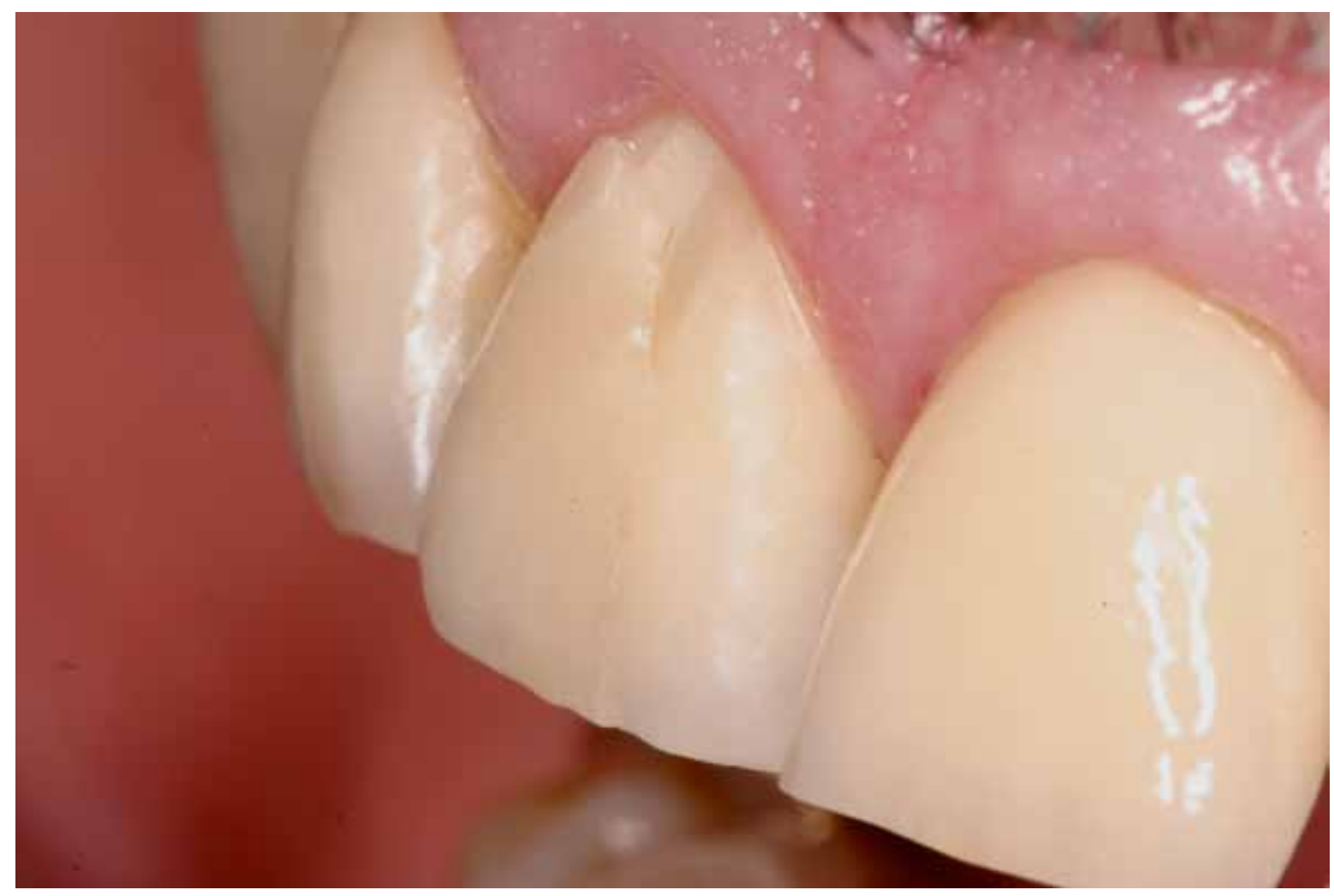

Abbildung 6: In-Ceram-Kronen auf den Zähnen 12-21 bei einem 67-jährigen Patienten nach 14 Jahren klinischer Verweildauer. Die Krone auf Zahn 11 zeigt einen vestibulären Riss mit Aussprengung der Verblendkeramik. Die meisten Frakturen der Verblendkeramik traten in dieser kohäsiven Art auf (ohne Exposition der Gerüstkeramik). 


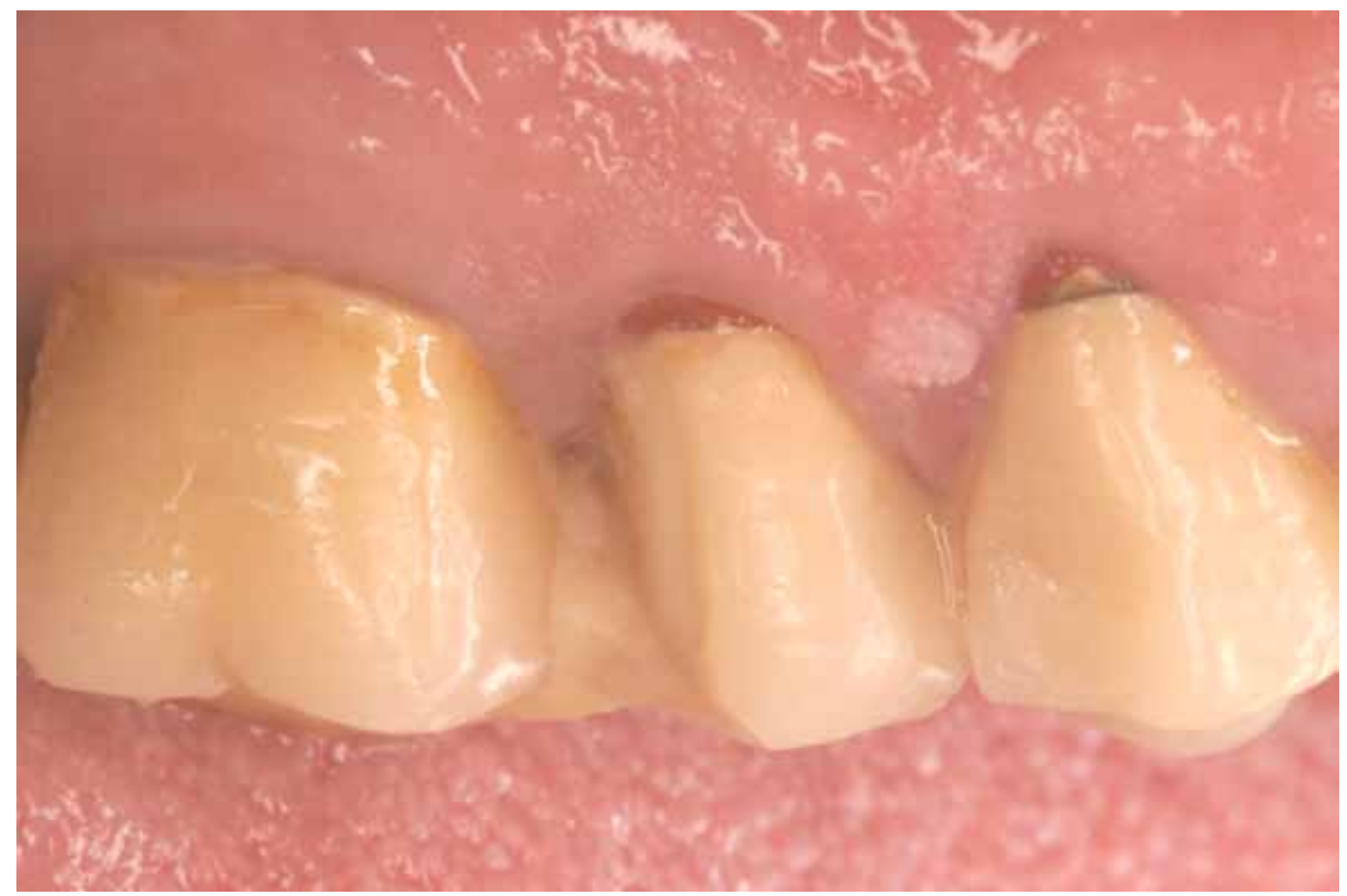

Abbildung 7: In-Ceram-Kronen auf den Zähnen 14-16 bei einem 70-jährigen Patienten nach 16 Jahren klinischer Verweildauer. Die Krone auf Zahn 15 zeigt eine vestibuläre Scherfraktur der Verblendkeramik, Zahn 14 weist eine initiale Randkaries auf. 


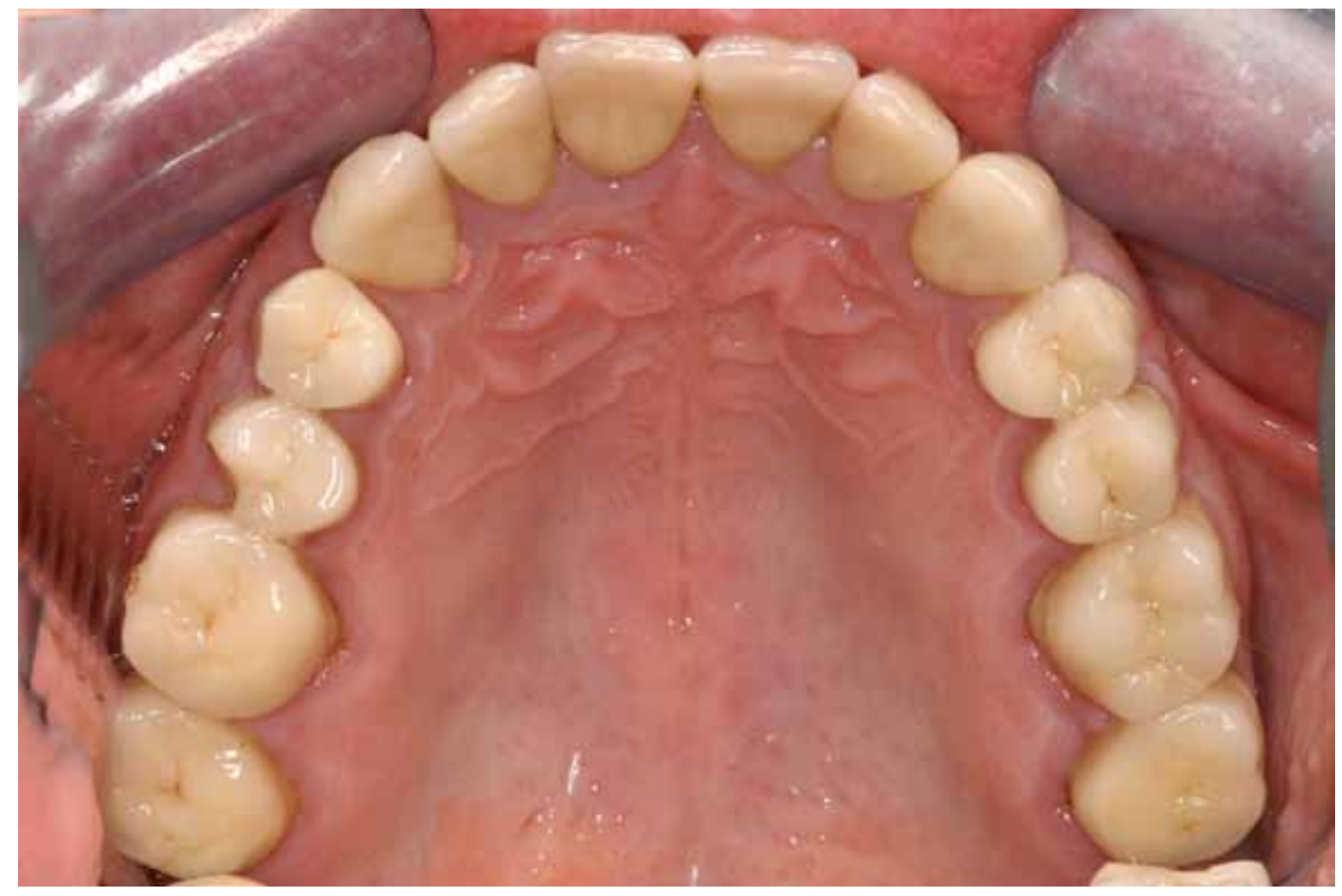

Abbildung 8: Komplettversorgung des Oberkiefers mit In-Ceram-Kronen (Patient identisch mit Abbildung 4) in der Aufsicht. Vestibulärer Defekt der Verblendkeramik ohne Exposition des Gerüstes an Zahn 15. 


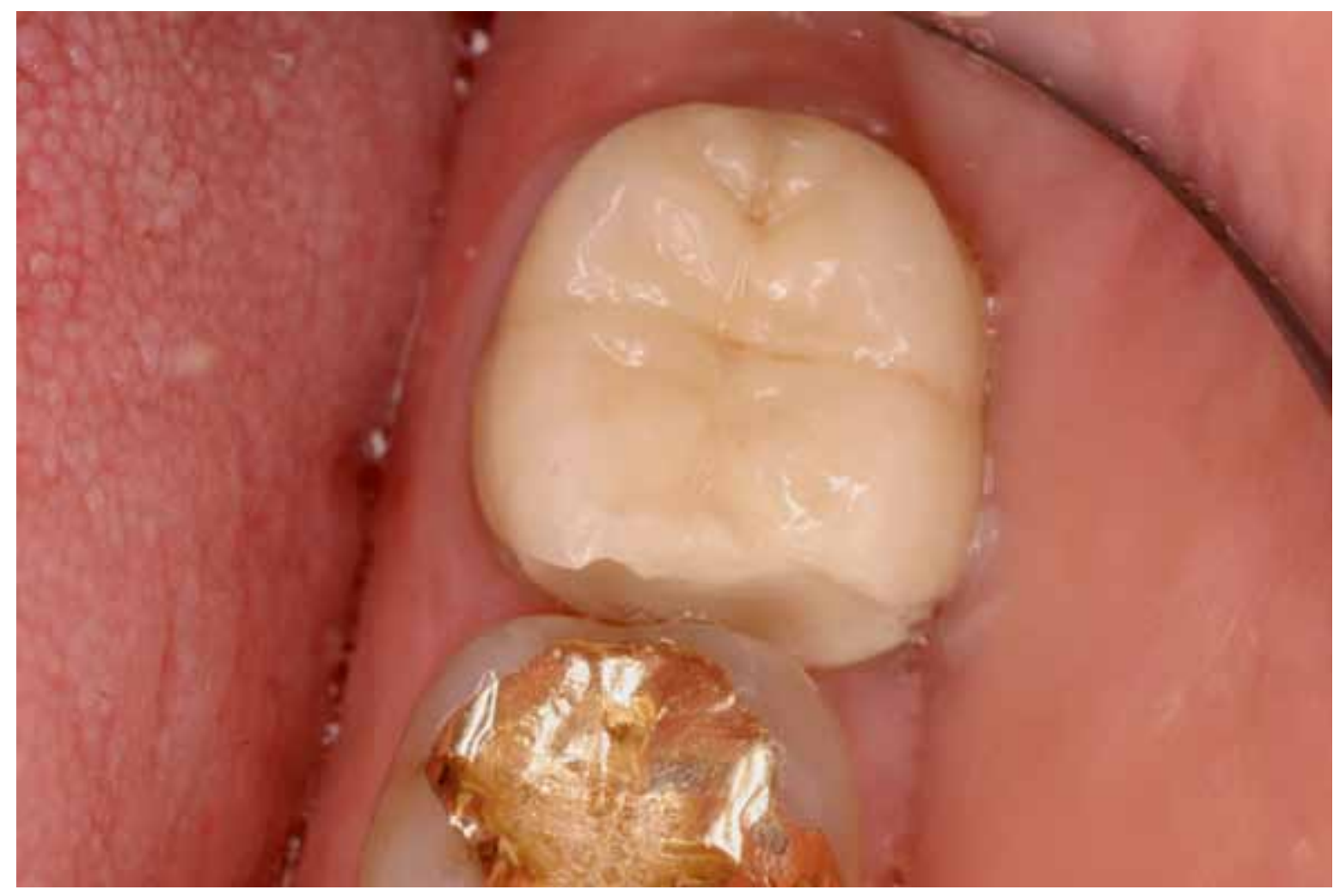

Abbildung 9: In-Ceram-Krone auf dem Zahn 38 bei einer 62-jährigen Patientin nach 14 Jahren klinischer Verweildauer. Die Krone zeigt eine approximale Scherfraktur der Verblendkeramik. Die Patientin beklagte eine immer wiederkehrende Retention von Speiseresten in diesem Bereich. 


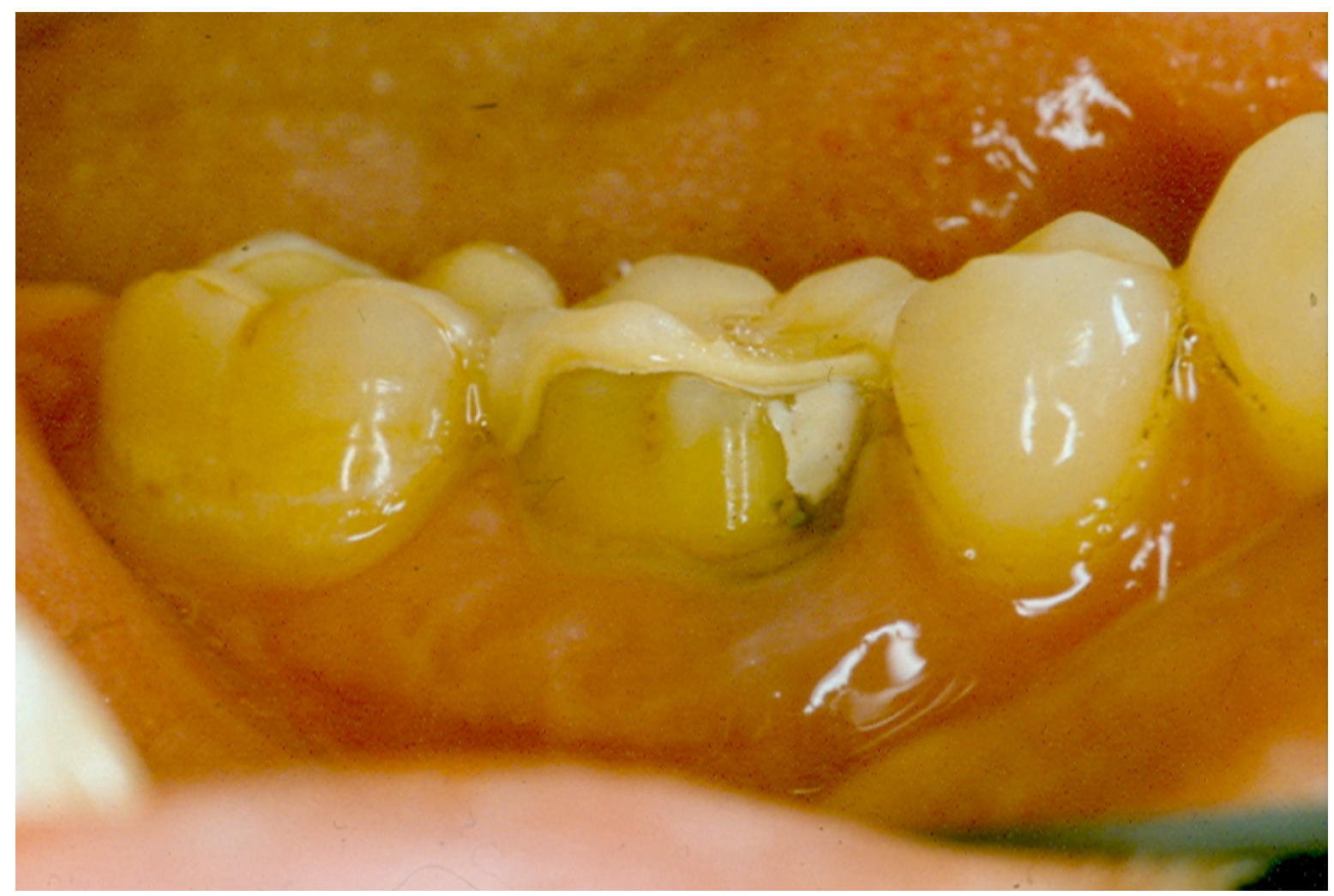

Abbildung 10: Gerüst-Fraktur einer In-Ceram-Krone auf dem Zahn 46 nach 12 Jahren klinischer Verweildauer im Bereich des zentrischen, tragenden Höckers im Unterkiefer. 


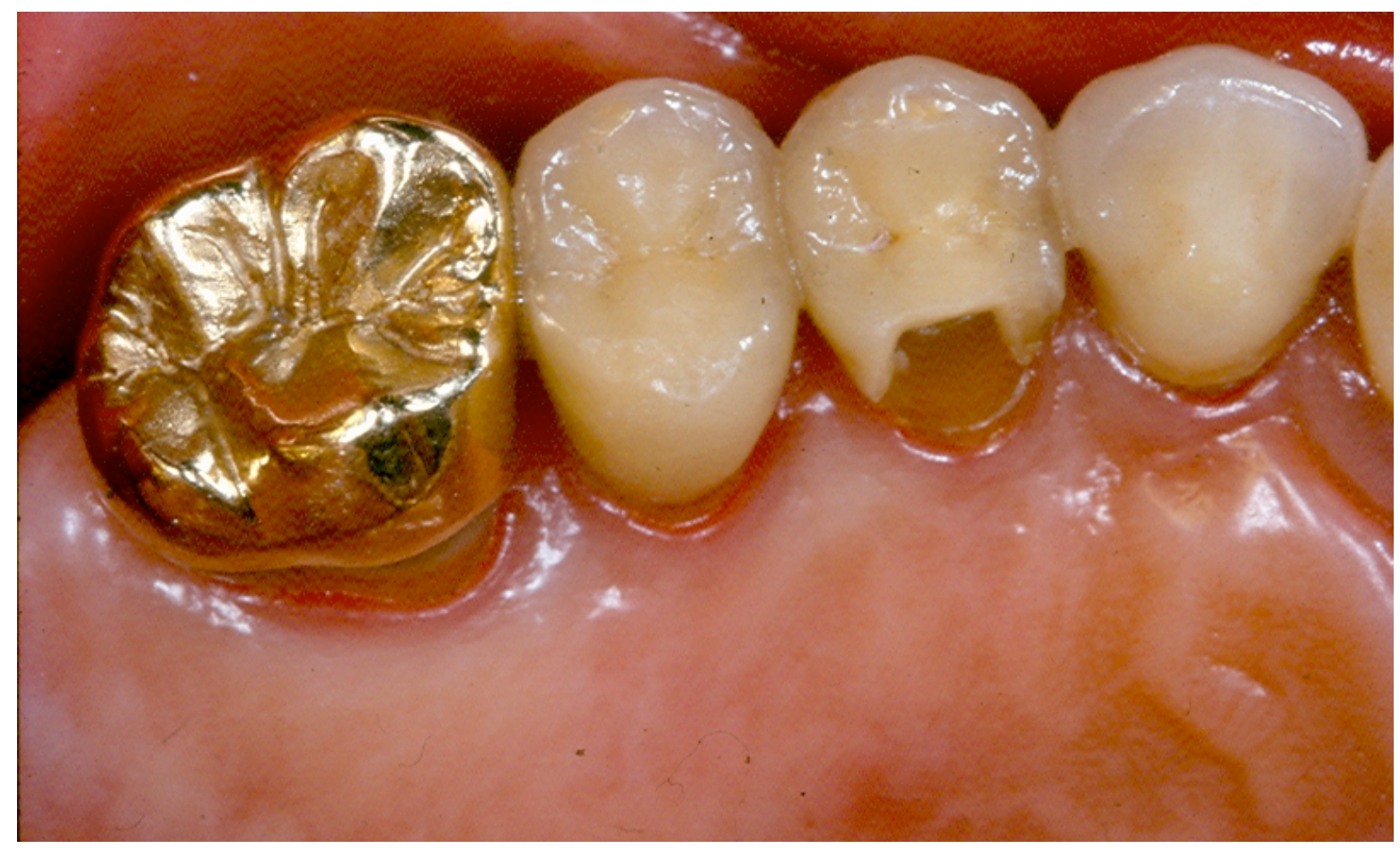

Abbildung 11: Gerüst-Fraktur einer In-Ceram-Krone auf dem Zahn 14 nach 13 Jahren klinischer Verweildauer im Bereich des zentrischen, tragenden Höckers im Oberkiefer. Die Mehrzahl der großflächigen Frakturen lagen im Bereich der tragenden (zentrischen) Höcker. Die In-Ceram-Kronen auf Zahn 13 und 15 erschienen intakt. 


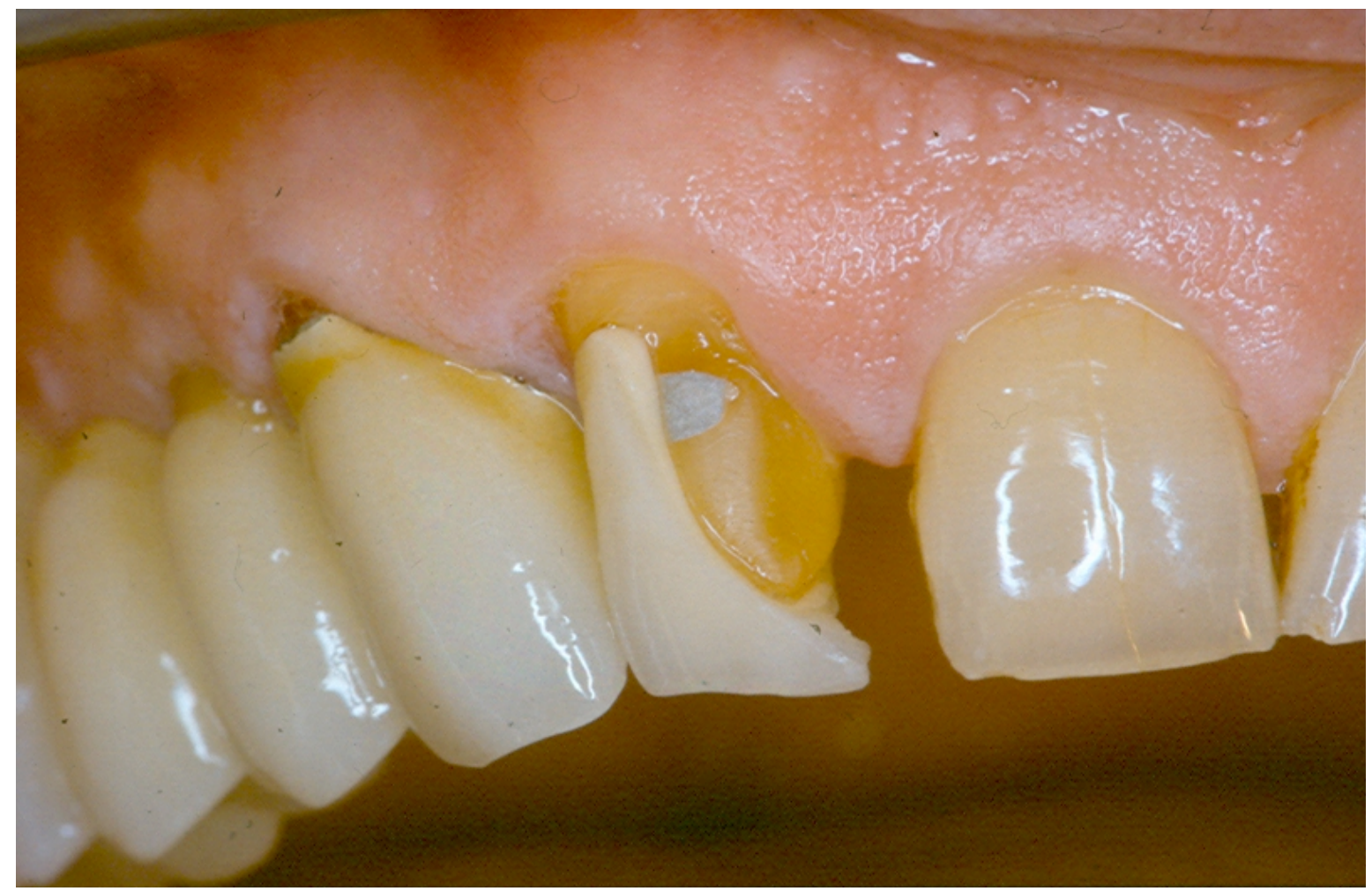

Abbildung 12: In-Ceram-Kronen auf den Zähnen 12-15 bei einem 78-jährigen Patienten nach 11 Jahren klinischer Verweildauer. An der Krone auf Zahn 12 zeigte sich eine Fraktur der Gerüstkeramik. Bei Zahn 13 wies der vestibuläre Kronenrand einen tastbaren Randspalt mit initialer Verfärbung auf. 


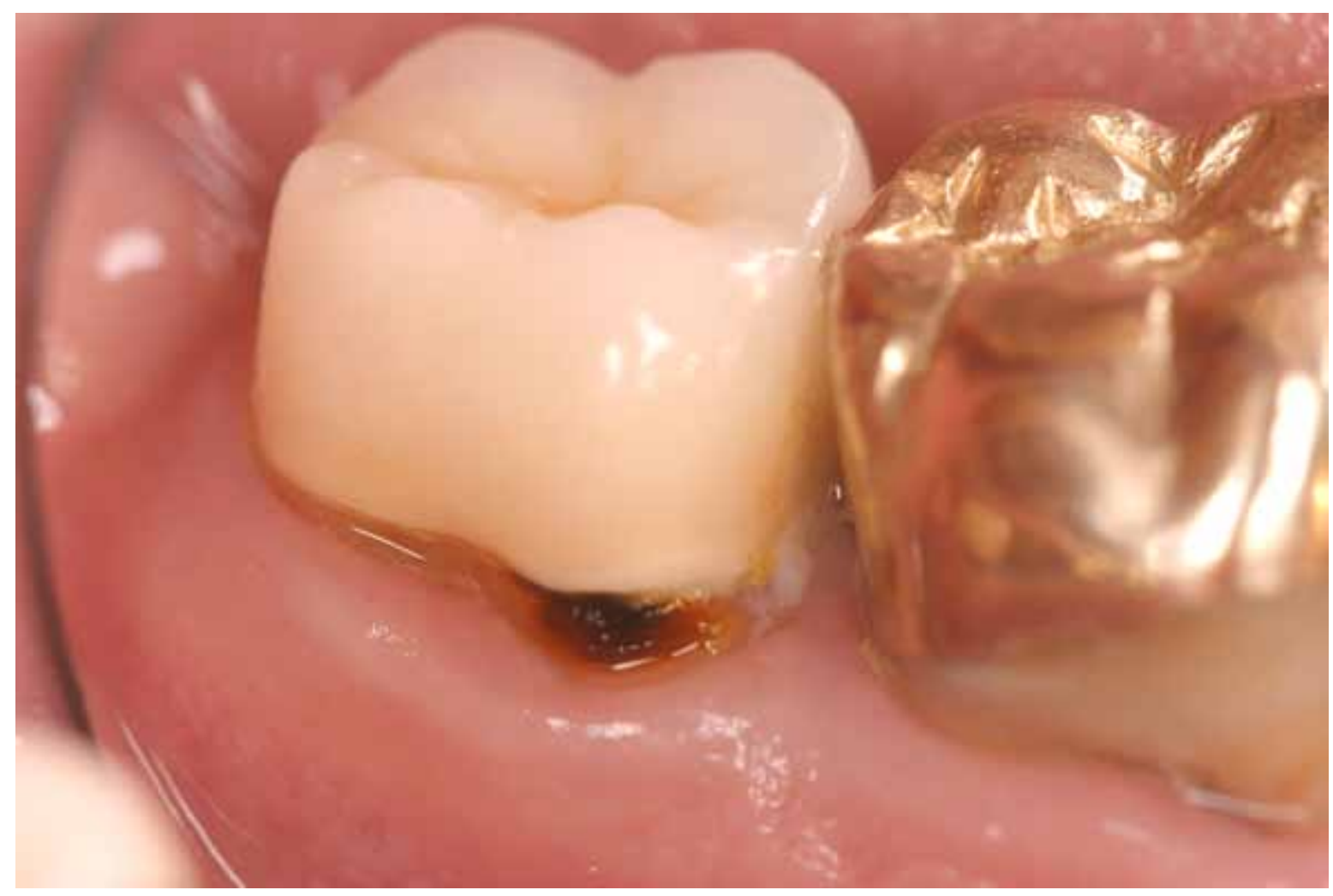

Abbildung 13: In-Ceram-Krone auf Zahn 37 bei einem 73-jährigen Patienten nach 11 Jahren klinischer Verweildauer. Der Kronenrand wies eine vestibuläre initiale Sekundärkaries auf. Mesial zeigte sich am etwas überkonturierten Kronenrand eine Plaque-Akkumulation, welche die Entstehung einer Sekundärkaries begünstigt haben könnte. 


\section{Diskussion}

In dieser Studie lag die Überlebensrate für anteriore und posteriore In-CeramEinzelzahnkronen nach 15 Jahren Beobachtungszeit bei 77,3\%. Bisher sind keine vergleichbaren Daten für glas-infiltrierte aluminiumoxidbasierte oder andere aktuelle vollkeramische Kronen über einen solchen Zeitraum verfügbar. Nur für glaskeramische Kronen gibt es klinische Langzeitdaten im 14-Jahres-Zeitraum. Hier konnte in einer Studie zu adhäsiv zementierten Glaskeramikkronen eine allgemeine Überlebensrate von 76\% gezeigt werden (Malament und Socransky, 1999). In dieser Studie wurden nur frakturierte Kronen als Verluste gewertet. Dies demonstriert die hohe technische Komplikationsrate glas-keramischer Kronen. In der vorliegenden Untersuchung wurden nur 12 von 41 Totalverlusten von Materialfrakturen verursacht. Basierend auf unterschiedlichen Überlebens-Kriterien scheint die LangzeitÜberlebensrate von konventionell zementierten In-Ceram-Kronen derer für adhäsiv zementierte Glaskeramik- und Feldspatkeramik-Kronen überlegen zu sein (Malament und Socransky, 1999; Sjogren et al., 1999).

Die Ergebnisse der vorliegenden Studie können außerdem mit Langzeit-Ergebnissen (durchschnittliche Beobachtungszeit: 10 Jahre) metallkeramischer Kronen verglichen werden. Auch in Anbetracht der unterschiedlichen Studien-Designs und definierten Restaurationscharakteristika wie bspw. „Überleben“ kann eine generelle Vergleichbarkeit in der Tendenz der Überlebensraten abgeleitet werden. Die meisten Untersuchungen von metallkeramischen Einzelzahnkronen legen Überlebensraten von über $80 \%$ bei Beobachtungszeiten bis zu 10 Jahren dar (Reitemeier et al., 2006; Bader und Shugars, 2009). Dies ist den 10-Jahres-Ergebnissen dieser Studie vergleichbar, bei welcher die Überlebensraten (Kaplan-Meier) um $89 \%$ und die Erfolgswahrscheinlichkeiten (Kaplan-Meier) um 84\% liegen und sowohl für den Front- als auch den Seitenzahnbereich tendenziell gleich gute Ergebnisse zeigen (Diagramm 13).

Es gibt nur wenige Studien, die Beobachtungszeiten über mehr als 10 Jahre beinhalten. In einer retrospektiven Studie über 1037 Vollkronen wurde eine Überlebensrate von 78\% nach 18 Jahren gezeigt. In dieser Untersuchung waren hauptsächlich biologische (66\%) gegenüber technischen Gründen für ein Versagen $\mathrm{zu}$ verzeichnen. Die häufigsten technischen Versagensgründe waren Keramik-Frakturen und Dezementierungen (DeBacker et al., 2006). In einer anderen Langzeit-Untersuchung 
von 50 Patienten mit 100 metallkeramischen Einzelzahnkronen wurde eine Überlebensrate von 78\% nach 20 Jahren demonstriert (Näpankangas und Raustia, 2008). Da der vorliegenden Studie die gleichen Kriterien zur Beurteilung der Überlebensraten zugrunde liegen, lässt sich vergleichend darstellen, dass die glasinfiltrierten alumniumoxid-basierten Einzelzahnkronen nach 15 Jahren ähnliche Ergebnisse aufweisen.

Allerdings zeigen nach 15 Jahren sowohl die zeitabhängigen Überlebens- als auch Erfolgsraten für den Front- und Seitenzahnbereich signifikant unterschiedliche Resultate (Diagramm 12). Diese lokalisationsbedingte Diskrepanz scheint ein spezifisches Problem der vollkeramischen Versorgungsform $\mathrm{zu}$ sein, da für metallkeramische Kronen auch in für diese langen Zeiträume identische Ergebnisse des klinischen Erfolges für den Front- und Seitenzahnbereich dargestellt werden konnten (Walton, 1999; DeBacker et al., 2006). Ein möglicher Grund hierfür könnte beispielsweise die Ermüdung der keramischen Gerüststruktur sein, welche für die Stabilität der Restauration entscheidend ist. Diese wird u.a. vom spezifischen Hydrolyse-Verhalten von Keramiken verursacht, die über einen langen Zeitraum dem Milieu der Mundhöhle ausgesetzt sind (Schwickerath und Coca, 1987). Des Weiteren ist bekannt, dass unter kontinuierlicher mechanischer und wechselnder thermischer Beanspruchung (wie im Patientenmund) in dentalen Keramiken im Laufe der Zeit fortlaufend Mikrorisse entstehen, welche die Integrität der Struktur insgesamt schwächen und zu makroskopischen Defekten führen können (Schwickerath, 1987). Derartige Versagensgründe können für metall-basierte Restaurationen weitgehend ausgeschlossen werden.

In Studien metallkeramischer Einzelzahnkronen und in der vorliegenden Untersuchung waren die häufigsten Gründe für ein Versagen biologischen Charakters (DeBacker et al., 2006; Reitemeier et al., 2006; Bader und Shugars, 2009). Dies demonstriert einen Unterschied zu früheren Studien zum klinischen Verhalten von vollkeramischen Kronen, in welchen Gerüstfrakturen mit $85 \%$ der Verluste die häufigsten Versagensgründe darstellten (Goodacre et al., 2003). In der vorliegenden Studie waren nur in 12 von 41 Fällen eine Gerüstfraktur oder eine großflächige Fraktur der Verblendkeramik ein Grund für das Versagen. Goodacre et al. (2003) kalkulierten eine durchschnittliche Frakturinzidenz mit 13\%. Aluminiumoxid-basierte Restaurationen der vorliegenden Untersuchung zeigten lediglich eine Frakturrate von 5\% nach einer durchschnittlichen Beobachtungszeit von 12 Jahren. Dies könnte ein 
Hinweis darauf sein, dass die verbesserten mechanischen Eigenschaften von glasinfiltrierten aluminiumoxidbasierten Restaurationen relativ $\mathrm{zu}$ konventionellen Glaskeramiken zu einer reduzierten Quote technischen Versagens geführt haben.

Smales und Hawthorne (1997) berichteten über eine Erfolgsrate (also Restaurationen ohne notwendige klinische Intervention nach Insertion) von 81\% nach 15 Jahren für goldbasierte metallkeramische Kronen. Diese Resultate sind konform mit den Ergebnissen einer anderen retrospektiven Studie, welche eine Erfolgsrate von $75 \%$ nach 20 Jahren für metallkeramische Einzelzahnkronen zeigt (Näpänkangas und Raustia, 2008). Die allgemeine Erfolgsrate der vorliegenden Untersuchung liegt bei ca. 69\% nach 15 Jahren klinischer Verweildauer. Dies bedeutet eine leicht erhöhte Komplikationsrate, welche hauptsächlich technischen Gründen zuzuordnen ist. 45\% der Komplikationen lagen als ein Chipping der Verblendkeramik vor, in den meisten Fällen als kohäsive Frakturen ohne Gerüstexposition. Dieser prozentuale Anteil liegt höher als bei metallkeramischen Kronen (Pjetursson et al., 2007; Näpänkangas und Raustia, 2008; De Backer et al., 2006). Dieser Befund findet sich in klinischen Studien zu aktuellen vollkeramischen Systemen wie den zirkoniumdioxidbasierten Restaurationen wieder, welche ebenfalls erhöhte „Chipping“-Quoten relativ zu metallkeramischen Versorgungen zeigen (Vult von Steyern et al., 2005; Sailer et al., 2007; Tinschert et al., 2007, Roediger et al., 2010). Aktuell postulierte Erklärungen für dieses Phänomen zielen zum einen auf den Aspekt der nicht ausreichend anatoformen Gerüstgestaltung und der resultierenden nicht-homogenen Stärke der Verblendkeramik ab. Eine höckerunterstützende Gerüstgestaltung dagegen vermeidet das Auftreten kritischer Zugspannungen in der Verblendung (Kern, 2008; Pospiech, 2010). Ein weiterer Erklärungsansatz ist die unterschiedliche Temperaturführung bei den Brennprozessen für metall- bzw. vollkeramische Restaurationen, was zu materialinternen Spannungen führen kann (Danzer et al., 2008). Sowohl FiniteElemente-Simulationsrechnungen (Eser et al., 2009) als auch erste in-vitro-Studien (Schmitter und Rammelsberg, 2009) haben einen positiven Effekt modifizierter Brennprogramme mit verlangsamten Aufheiz- und Abkühlungsphasen gezeigt, was allerdings in klinischen Langzeitstudien bestätigt werden muss. Wahrscheinlich sind diese Hinweise auch zur Verbesserung des „Chipping“-Verhaltens von In-CeramRestaurationen anwendbar. Außerdem ist bekannt, dass vollkeramische Versorgungen im Allgemeinen sensibler auf Präparationsfehler, wie unzureichende Abstützung der Restauration im marginalen Bereich oder auf exzessives Einschleifen mit Frakturen 
reagieren (Kerschbaum et al., 2009; Pospiech, 2010). Ein weiterer aktueller Lösungsansatz findet sich in dem Bestreben, verblendungsfreie vollkeramische Restaurationen aus Hartkernkeramiken zu gestalten. Erste Fallstudien zeigen hier positive Ergebnisse (Schneider et al., 2010). Aufgrund der relativen geringen Anzahl von Chipping-Fällen ließ sich allerdings keine signifikante, lokalisationsbedingte Häufung ableiten.

Ein Retentionsverlust lag in 3 Fällen $(1,3 \%)$ vor. Das entspricht in etwa den Ergebnissen vorangegangener Studien, in welchen diese Rate mit 2\% angegeben worden ist (Goodacre et al., 2003). Obwohl die hier gezeigte Inzidenz für Dezementierungen als niedrig einzustufen ist, kann dieser Aspekt Hinweis auf die marginale und interne Passungsqualität einer Restaurationsform sein (Moldovan, 2007). Das gleiche gilt für ein Auftreten von marginaler Sekundärkaries, dem im Rahmen dieser Studie in insgesamt 10 Fällen mit einer adhäsiven Versiegelung begegnet werden musste. Beim Vergleich dreier vollkeramischer Systeme zeigte das In-Ceram-System mit seinen Werten für einen marginalen Randspalt $(112 \pm 55 \mu \mathrm{m})$ relativ die größten Ungenauigkeiten (Yeo et al., 2003), wobei ein Randspalt von 120 $\mu \mathrm{m}$ allerdings allgemein als akzeptabel gilt (McLean und von Fraunhofer, 1971). Ein weiterer ätiologischer Faktor für die Entstehung von marginaler Randkaries könnten allerdings auch überkonturierte Kronenränder sein, welche die kariesfördernde Plaqueretention begünstigen. Dies kann beim In-Ceram-System durch die erweiterte Raumforderung der Restauration v.a. dann entstehen, wenn die Präparation marginal eine nicht ausreichende Schnitttiefe aufweist.

Die Überlebensrate anteriorer Kronen nach 15 Jahren entspricht 85\% gegenüber $64,6 \%$ für posteriore Einzelzahnkronen. Lediglich $54,2 \%$ der posterioren Restaurationen blieben ereignisfrei über den Beobachtungszeitraum von 15 Jahren (74,5\% für anteriore Restaurationen).

Auch bei abschließender ökonomischer Bewertung vollkeramischer Restaurationen ist anzumerken, dass sich die Konditionen zu ihren Gunsten verschoben haben (Kern, 2008). Zum einen bieten moderne Herstellungsverfahren, wie die CAD/CAMTechnologie, Zeit einsparende Potentiale, zum anderen wurde die preisliche Mehrbelastung des Patienten mittlerweile durch den hohen Preis für Edelmetalllegierungen relativiert. 


\section{5-Jahres-Ergebnisse}

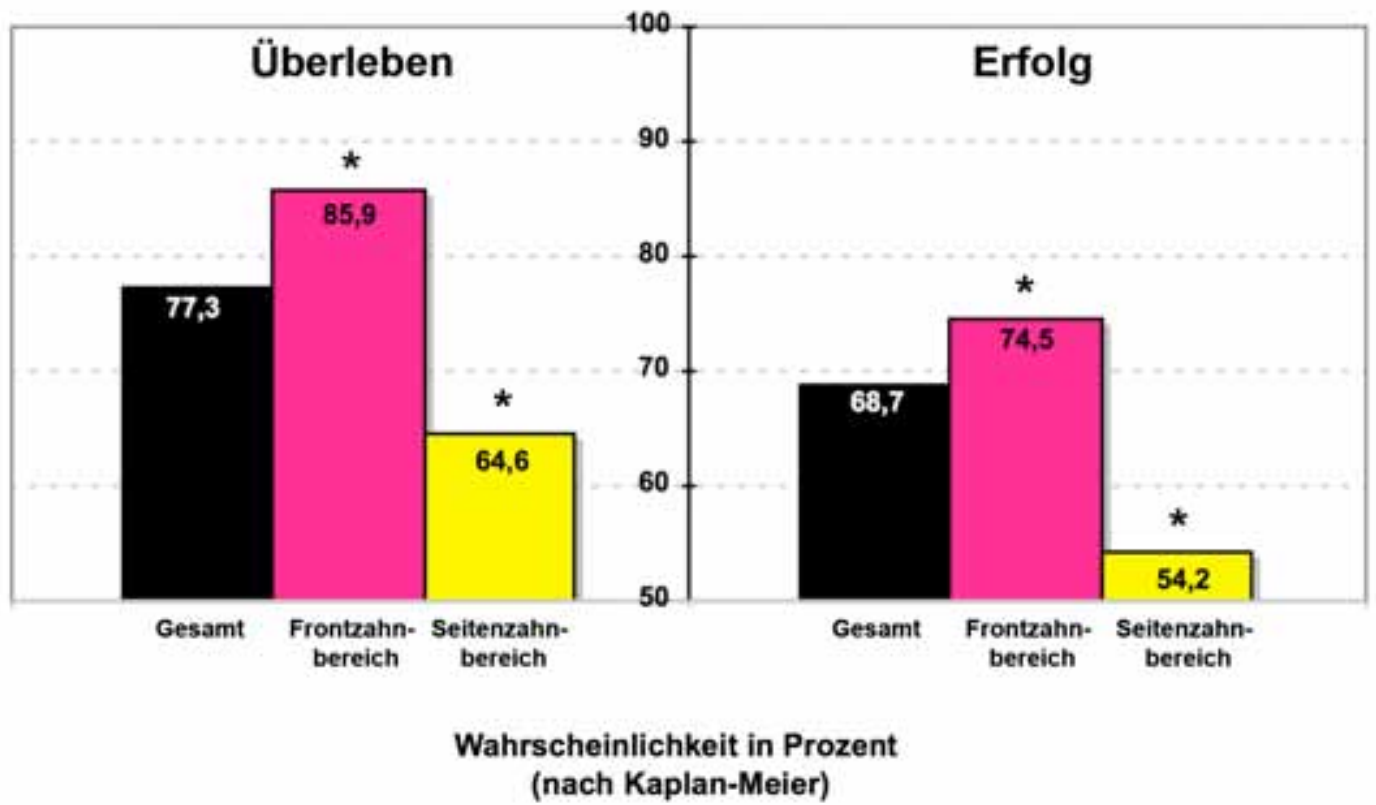

Diagramm 12: Darstellung der klinischen Überlebens- und Erfolgswahrscheinlichkeit von InCeram-Einzelzahnkronen für 15 Jahre Verweildauer nach Kaplan-Meier. Die unterschiedlichen Ergebnisse im Front- und Seitenzahnbereich zeigten jeweils statistische Signifikanz.

Nach Analyse dieser Daten lässt sich feststellen, dass das klinische Langzeitverhalten glas-infiltrierter aluminiumoxidbasierter Einzelzahnkronen abhängig von der Lokalisation im Mund ist. Die Analyse mit Hilfe des Log-Rang Testes zeigte eine signifikant bessere zeitabhängige Überlebens- $(p=0,0239)$ und Erfolgsrate $(0,004)$ anteriorer Restaurationen für 15 Jahre klinischer Liegedauer. Dies stellt einen Unterschied im Vergleich mit metallkeramischen Kronen dar, deren klinisches Langzeitverhalten im anterioren und im posterioren Bereich identisch ist. Zwei Studien zum klinischen Langzeitverhalten metallkeramischer Einzelzahnkronen (Walton, 1999; DeBacker et al., 2006) zeigten keinen signifikanten Einfluss der Lokalisation im Mund auf. Die Überlebensrate von anterioren In-CeramEinzelzahnkronen ist äquivalent $\mathrm{zu}$ denen metallkeramischer Kronen nach 15-20 Jahren klinischer Beobachtungszeit in retrospektiven Studien mit denselben Kriterien zur Überlebenswahrscheinlichkeit (DeBacker et al. 2006, Näpänkangas and Raustia 2008). Verglichen mit diesen Ergebnissen sind die klinischen Langzeitergebnisse posteriorer In-Ceram-Einzelzahnkronen in diesem Zeitraum offensichtlich etwas unterlegen, wohingegen sie bis zu 10 Jahren Verweildauer äquivalent erscheinen. 


\section{0-Jahres-Ergebnisse}

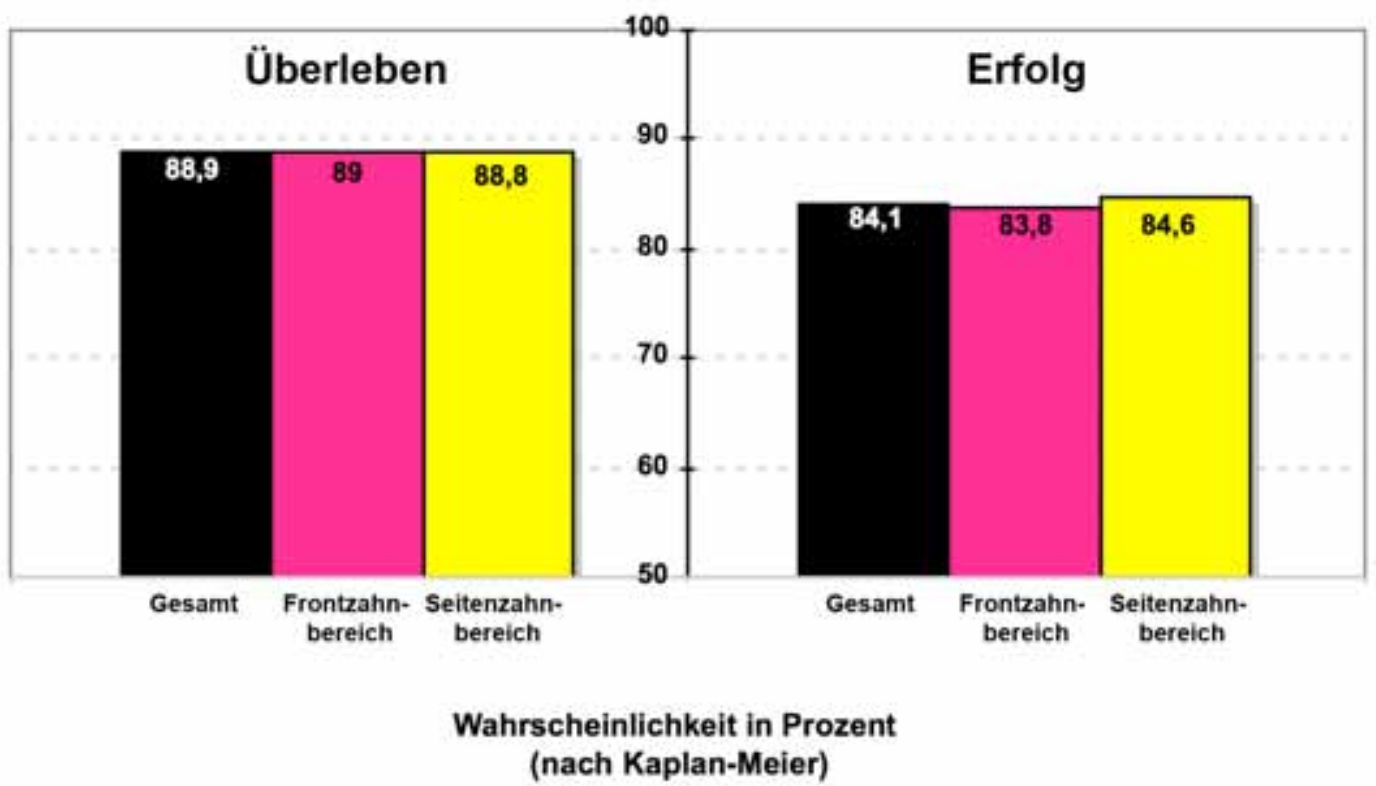

Diagramm 13: Darstellung der klinischen Überlebens- und Erfolgswahrscheinlichkeit von InCeram-Einzelzahnkronen für 10 Jahre Verweildauer nach Kaplan-Meier.

Im klinischen Alltag zeigen bei zahnärztlich-prothetischen Restaurationen die Pfeilerzähne, welche zum Versorgungszeitpunkt devital und endondontisch versorgt waren, teilweise erhöhte Misserfolgsquoten. In dieser Studie waren zum Zeitpunkt der prothetischen Versorgung 34 von 229 Zähnen bereits endodontisch versorgt (ca. 15\%), die fehlende Vitalität war allerdings kein Kausalfaktor für eine erhöhte Komplikations- oder Verlustrate. Möglicherweise spielt die Vitalität der Pfeilerzähne bei der Verankerung größerer prothetischer Konstruktionen wie Brückenzahnersatz oder kombiniert festsitzend-herausnehmbarem Zahnersatz eine entscheidende Rolle, da die mechanische Beanspruchung des Pfeilerzahnes in diesen Fällen stark erhöht ist (Wegner et al., 2006), im Gegensatz zu Einzelzahnversorgungen wie in der vorliegenden Studie. Dies entspricht den Resultaten anderer Studien zu vollkeramischen Einzelzahnversorgungen (Malament und Socransky, 1999).

Des Weiteren muss eine von der Restzahnhartsubstanz abhängige eingeschränkte klinische Anwendbarkeit relativ zur metallkeramischen Alternative erwähnt werden. Dies liegt in den besonderen Anforderungen bei der Präparation des Pfeilerzahnes begründet.

Die Resultate der vorliegenden Studie unterstützen die Ergebnisse einer systematischen Literatur-Analyse von Versagens- und Komplikationsraten von 
Einzelzahnkronen (Pjetursson et al., 2007). Ein Rückschluss aus dieser Arbeit ist, dass die Überlebensraten vollkeramischer Einzelzahnkronen, appliziert im Frontzahnbereich, hohe klinische Überlebensraten, vergleichbar mit dem „Goldstandard“ der metallkeramischen Kronen, aufweisen. Im Seitenzahnbereich allerdings sind die Überlebensraten für In-Ceram- und glaskeramische Kronen bei klinischen Verweildauern über 10 Jahre signifikant niedriger als die für metallkeramische Kronen. Obwohl diese Literatur-Analyse lediglich auf Studien mittelfristiger Beobachtungszeiten beruht, geben die Resultate ein konsistentes Bild zu den Langzeitergebnissen dieser Studie für glas-infiltrierte aluminiumoxid-basierte Einzelzahnkronen ab.

Die Ergebnisse der vorliegenden Untersuchung sollten allerdings unter Berücksichtigung der typischen Limitationen eines retrospektiven Studiendesigns interpretiert werden. Ein Problem retrospektiver Studien ist die Verfügbarkeit analysierbarer, konsistenter Daten. Allerdings stellte dies hier konkret kein Problem dar, da der klinische Verlauf in der Abteilung für zahnärztliche Prothetik seit Beginn der Versorgung 1991 im Rahmen einer standardisierten Prozedur dokumentiert worden ist. Deshalb kann hier davon ausgegangen werden, dass die vorliegenden Daten repräsentativ und vergleichbar sind.

Eine andere spezifische Einschränkung einer retrospektiven Studie, welche eine Nachsorge-Untersuchung beinhaltet, ist, dass Störungen in Bezug auf das Bevölkerungssegment, welches an der Studie teilgenommen hat, nur verallgemeinert werden können (Haselton et al., 2000). Von den 198 Patienten, welche kontaktiert wurden, haben 138 nicht teilgenommen, was in einer Rücklauf-Quote von 30,3\% resultierte. Für die Nicht-Teilnahme wurden verschiedene Gründe aufgenommen. Die häufigsten waren weit entfernte Umzugsorte, schwere Krankheit oder Tod des Patienten, sicherlich bedingt durch den sehr langen Dokumentationszeitraum. Außerdem muss berücksichtigt werden, dass eventuell hauptsächlich zufriedene Patienten zur Teilnahme an der Nachuntersuchung bereit waren, was unter Umständen zu einer positiven Überschätzung der Überlebens- und Erfolgsraten geführt haben könnte.

Dennoch kann festgestellt werden, dass in Anbetracht der vorliegenden Bedingungen, wie den typischen klinischen Konditionen (Bsp.: verschiedene Behandler), der langfristigen Beobachtungsdauer und der großen Zahl von involvierten 
Pfeilerzähnen/Restaurationen sehr valide Daten generiert werden konnten, die entsprechend fundierte Schlussfolgerungen zulassen.

Auch unterstreichen die dargestellten Resultate des klinischen Langzeiterfolges speziell im Seitenzahnbereich (relativ zum Frontzahnbereich) in der 10- bzw. 15Jahres-Auswertung den Wert einer solchen klinischen Nachuntersuchung. Nur der ungewöhnlich lange Nachverfolgungszeitraum der Restaurationen von 15 Jahren offenbarte zeitabhängige Kriterien für eine derart differenzierte Bewertung vollkeramischer aluminiumoxid-basierter glas-infiltrierter Einzelzahnkronen im Seitenzahnbereich.

\begin{tabular}{|c|c|c|}
\hline Klasse & Ausmaß & Konsequenz \\
\hline 1 & minimal, kaum sichtbar & Polieren \\
\hline 2 & mit bloßem Auge erkennbar, nicht funktionsrelevant & Polieren \\
\hline 3 & $\begin{array}{c}\text { Frontzahnbereich: mit bloßem Auge erkennbar, } \\
\text { ästhetisch relevant, formverändernd }\end{array}$ & ggf. Reparatur mit Komposit \\
\hline 4 & $\begin{array}{l}\text { Seitenzahnbereich: mit bloßem Auge erkennbar, } \\
\text { im okklusionstragenden Bereich }\end{array}$ & $\begin{array}{l}\text { ggf. Reparatur mit Komposit, } \\
\text { je nach Ausmaß auch Ersatz }\end{array}$ \\
\hline 5 & $\begin{array}{l}\text { massive Chippings, } \\
\text { auch weitergehende kleinere Chippings }\end{array}$ & $\begin{array}{l}\text { Austausch oder Reparatur } \\
\text { mittels adhäsiver Befestigung } \\
\text { des erhaltenen Chips oder } \\
\text { eines Keramikveneers }\end{array}$ \\
\hline
\end{tabular}

Tabelle 3: $\quad$ Chipping-Klassifikation nach Pospiech (2010) 


\section{Schlussfolgerungen}

1. Die Überlebens- und die Erfolgsraten von In-Ceram-Einzelzahnkronen im 15-JahresZeitraum werden durch deren Lokalisation im Mund (Front- gegenüber Seitenzahnbereich) signifikant beeinflusst. Anteriore Restaurationen zeigen sich klinisch erfolgreicher als posterior inserierte In-Ceram-Einzelzahnkronen.

2. Die Langzeit-Überlebens- und Erfolgsraten von glasinfiltrierten aluminiumoxidbasierten Einzelzahnkronen im Frontzahnbereich sind denen für metallkeramische Kronen (VMKs) publizierten Raten nahezu äquivalent. Da diese Restaurationsform aufgrund der guten klinischen Langzeiterfahrungen aktuell noch als Referenz gewertet werden, kann hieraus eine Empfehlung zur klinischen Anwendbarkeit von In-CeramEinzelzahnkronen im Frontzahnbereich abgeleitet werden.

3. Die Überlebens- und Erfolgsraten von aluminiumoxidbasierten In-CeramEinzelzahnkronen im Seitenzahnbereich sind denen im Frontzahnbereich und denen der VMK-Restaurationen im 15-Jahres-Zeitraum unterlegen. Hier sollte die Indikationsstellung kritisch abgewogen werden.

4. Die häufigsten Ursachen für ein Totalversagen hatten einen biologischen Hintergrund. Hier sind kariologische, endodontische und parodontologische Faktoren zu nennen.

5. Die häufigste technische Komplikation trat in Form von Frakturen der Keramik auf. Zum einen waren dies Frakturen der Verblendkeramik unterschiedlichen Schweregrades und zum anderen Brüche des Aluminiumoxid-Gerüstes.

6. Die Vitalität des Pfeilerzahnes bei Inkorporation der Restauration hatte keinen Einfluss auf die Komplikations- und Verlustrate.

7. In manchen Fällen (häufiger bei Wiederholungsüberkronungen) kann eine In-CeramVersorgung aufgrund des reduzierten Hartsubstanzangebotes nicht durchgeführt werden. Hier sollte der substanzschonenden metall- oder zirkoniumdioxid-basierten Variante der Vorzug gegeben werden. 


\section{$7 \quad$ Zusammenfassung}

Glas-infiltrierte aluminiumoxid-basierte Restaurationen (In-Ceram) werden seit über 20 Jahren klinisch angewendet. Allerdings sind bis heute die Ergebnisse zur Erfolgswahrscheinlichkeit dieser Art von Zahnersatz nur mit klinischen Verweildauern mittlerer Größenordnung (über mindestens 3 Jahre) verfügbar. Diese retrospektive Untersuchung evaluiert die Langzeitüberlebensrate von konventionell zementierten InCeram-Einzelzahnkronen mit einer maximalen Beobachtungszeit von 17,7 Jahren (mittlere Beobachtungszeit 12,8 Jahre) sowie den Einfluss der Zahnposition auf die Überlebens- und Erfolgsrate dieser Restaurationsform.

Insgesamt 60 Patienten (31 Frauen und 29 Männer) der Abteilung Prothetik der Universitätszahnklinik in Göttingen wurden im Rahmen dieser Studie nachuntersucht. Diese Patienten haben insgesamt 229 In-Ceram-Kronen in den Jahren 1991-1999 erhalten, wovon 129 im Frontzahnbereich und 100 im Seitenzahnbereich inseriert wurden. Zum einen wurden die zeitabhängige Überlebensrate (in-situ-Kriterium) und zum anderen die Erfolgsrate (ereignisfreie Restaurationen) nach Kaplan-Meier ermittelt.

In insgesamt 41 Fällen war es zu einem Verlust der Restauration gekommen. Daraus ergibt sich eine Überlebensrate von 77,3\% nach 15 Jahren. Die Erfolgsrate betrug 68,9\%. Von den 129 Frontzahnkronen gingen 17 verloren. Dies entspricht einer Überlebensrate von 85,9\% nach 15 Jahren. Im anterioren Bereich ergab sich eine Erfolgsrate von $74,5 \%$. Für den Seitenzahnbereich wurden 14 Verluste festgestellt, dies entspricht einer Überlebenswahrscheinlichkeit von 64,6\% nach 15 Jahren. Die Erfolgswahrscheinlichkeit betrug hier 54,2\%. Der durchgeführte Log-Rang-Test offenbarte einen signifikanten Einfluss der Lokalisation der Kronen im Mund auf ihre Überlebensrate und ihre Erfolgswahrscheinlichkeit.

Die klinischen Erfolgsraten von In-Ceram-Kronen nach 15 Jahren im Frontzahnbereich sind denen von Verblendmetallkeramik-Kronen (VMKs) vergleichbar, welche für diese Indikation als Standard gelten. Signifikant schlechtere Überlebens- und Erfolgsraten von In-Ceram-Kronen konnten im Seitenzahnbereich eruiert werden. Die meisten Verluste wurden durch biologische Komplikationen verursacht, die häufigste technische Misserfolgs-Ursache waren Frakturen der Verblendkeramik. In manchen klinischen Fällen ist aufgrund eines reduzierten Hartsubstanzangebotes eine Versorgung mit In-Ceram-Restaurationen nicht möglich. 


\section{$8 \quad$ Literaturverzeichnis}

Adamcyk A, Spiechowicz E (1990):

Plaqueaccumulation and crowns made of various materials.

Int J Prosthodont $\underline{3}, 285-291$

Bader JD, Shugars DA (2009):

Summary review of the survival of single crowns.

Gen Dent $\underline{57,74-81}$

Boysen H, Frey F, Vogt T (1991):

Neutron Powder Investigation of the Tetragonal to Monoclinic Phase Transformation in Undoped Zirconia.

Acta Cryst $\underline{47}, 881$

Chan C, Weber H (1986):

Plaqueretention and teeth restored with full-ceramic crowns: A comperative study. J Prosthet Dent 56,666-671

Danzer R, Lube T, Supancic P, Damani R (2008):

Fracture of ceramics.

Adv Eng Mater 10,275-298

De Backer H, Van Maele G, De Moor N, Van den Berghe L, De Boever J (2006):

An 18-year retrospective survival study of full crowns with or without posts.

Int J Prosthodont 19,136-142

Erpenstein H, Kerschbaum Th (1995):

Frakturanfälligkeit von glas- und galvanokeramischen Kronen.

Dtsch Zahnärztl Z 50,668-670 
Eser A, Bezold A, Wiesner CV, Vollmann M (2009):

Optimization of residual stresses on all-ceramic fixed partial dentures using Finite Element Analysis with viscoelastic material modelling. Interner Forschungsbericht DeguDent. Anwenderbrief Cercon 1/2009

Fischer J, Schmid M, Kappert HF, Strub JR (1991):

Gefügeausbildung der dentalkeramischen Kernmasse In-Ceram und thermische Dehnung ihrer Einzelkomponenten.

Dtsch Zahnärztl Z $\underline{46}, 461-463$

Friedman S:

Introduction to ceramics and glasses.

In: ASM Engeneering Materials Handbook, Vol. 4: Ceramic and glasses.

ASM International, Philadelphia 1991,1-40

Goodacre CJ, Bernal G, Rungcharassaeng K, Kann JYK (2003):

Clinical Complications in fixed prosthodontics.

J Prosthet Dent $\underline{\text { 90,31-41 }}$

Hahn R, Löst C (1992):

Konventionelle Dentalporzellane versus bruchzähe Hochleistungskeramiken.

Dtsch Zahnärztl Z 47,659-664

Haselton DR, Diaz-Arnold AM, Hillis SL (2000):

Clinical assessment of high-strenght all-ceramic crowns.

J Prosthet Dent $\underline{83}, 396-401$

Hellwig E, Klimek J, Attin Th:

Einführung in die Zahnerhaltung, 2.Auflage,Urban \& Fischer München 1999,S.28-29

Hofer B (1984):

Anwendung des HIP Verfahrens bei keramischen Werkstoffen.

Keram Z 36,552 
Hüls A (1995):

Zum Stand der klinischen Bewährung infiltrationskeramischer Verblendkronen.

Dtsch Zahnärztl Z $\underline{50}, 674-676$

Kaplan M, Meier P (1958):

Nonparametric estimation from incomplete observation.

J Am Stat Assoc $\underline{53}, 457-465$

Kappert HF (1996):

Vollkeramik. Werkstoffkunde- Zahntechnik- Klinische Erfahrung.

Quintessenz Verlag Berlin,193-210

Kappert HF (1996):

Vollkeramik. Werkstoffkunde- Zahntechnik- Klinische Erfahrung.

Quintessenz Verlag Berlin,225-230

Kappert HF, Ulbrich J, Gläss P, Huber H, Neumüller H (1989):

Determining the threshold level for sensibility responses to galvanic potentials in the oral cavity.

Dtsch Zahnärztl Z $\underline{44}, 50-52$

Kappert HF, Schwickerath H, Veiel T, Bregazzi J (1994):

Zur Korrosionsfestigkeit aufbrennfähiger Edelmetallegierungen.

Dtsch Zahnärztl Z $\underline{49}, 716-721$

Kappert HF, Knipp U, Wehrstein A, Kmitta M, Knipp J (1995):

Festigkeit von Zirkonoxid-verstärkten Keramikbrücken aus In-Ceram.

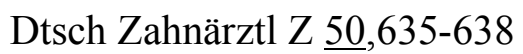

Kern M (2008):

Vollkeramik- klinisch und praktisch bewährt?

Dtsch Zahnärztl Z 63,145-150 
Kern M (2010):

Longitudinal bewährt - Tagungsbericht des 9. Keramik-Symposiums in München Dtsch Zahnärztl Z $\underline{65}, 115-119$

Kerschbaum T, Faber FJ, Noll FJ, Keiner M, Hürther W, Schumacher S, Keller E (2009):

Komplikationen von Cercon-Restaurationen in den ersten fünf Jahren.

Dtsch Zahnärztl Z $\underline{64}, 81-89$

Körber KH, Ludwig K, Dünner P (1984):

Experimental studies of the abrasion effect between dental enamel and dental ceramic. Dtsch Zahnärztl Z $\underline{\text { 39, }}$,-11

Luthardt R, Herold V, Sandkuhl O, Reitz B, Knaak JP, Lenz E (1998):

Kronen aus Hochleistungskeramik. Zirkondioxid-Keramik, ein neuer Werkstoff in der Kronenprothetik.

Dtsch Zahnärztl Z 53,280-285

Maier HM:

Leitfaden technische Keramik. Werkstoffkunde II

Keramik. Selbstverlag. Institut für keramische Komponenten im Maschinenbau, Aachen 1991

Malament KA, Socransky SS (1999):

Survival of Dicor glass-ceramic dental restorations over 14 years: Part I. Survival of Dicor complete coverage restorations and effect of internal surface acis etching, tooth position, gender and age.

J Prosthet Dent $\underline{81}, 23-32$

Malament KA, Socransky SS (1999):

Survival of Dicor glass-ceramic dental restorations over 14 years: Part II: Effect of thickness of Dicor material and design of tooth preparation.

J Prosthet Dent 81,662-667 
Marx R, Meiners H:

Taschenbuch der zahnärztlichen Werkstoffkunde.

5.überarbeitete und ergänzte Auflage.

Dtsch Zahnärzteverlag, Köln 2001,S.169

McLean JW, von Fraunhofer JA (1971):

The estimation of cement film thickness by an in vivo technique.

Br Dent J 131, 107-111

McLean JW (1978):

Wissenschaft und Kunst der Dentalkeramik.

Berlin, Chicago, Rio de Janeiro, Tokio

Moffa JP, Lugassy AA, Ellison JA (1988):

Clinical evaluation of a custable ceramic material. Three-Year-Study.

J Dent Res $\underline{67}, 118, A b s t r$ No43

Moldovan O: Innovatives Modell für die In-vitro-Untersuchung der internen und externen 3D-Passgenauigkeit CAM-gefertigter Zirkondioxid-Brückengerüste.

Med. Diss. Göttingen 2007

Näpänkangas R, Raustia A (2008):

Twenty-year follow-up of metal-ceramic single crowns: a retrospective study.

Int J Prosthodont $\underline{4}, 307-311$

Pjetursson BE, Sailer I, Zwahlen M, Hämmerle CH (2007):

A systematic review of the survival and complication rates of all-ceramic and metalceramic reconstructions after an observation period of at least 3 years. Part I: Single crowns.

Clin Oral Implants Res $\underline{3}, 73-85$ 
Pospiech P: Vollkeramische Restaurationen: Werkstoffkundliche und klinische Aspekte, Keramische Werkstoffe. In: Zahnärztliche Prothetik. Gernet W, Biffar R, Schwenzer N, Ehrenfeld M 3.vollständig überarbeitete und erweiterte Auflage, Thieme-Verlag, Stuttgart 2007,71-79

Pospiech P (2010):

Chipping - systemimmanente oder verarbeitungsbedingte Probleme?

Quintessenz $\underline{61(2)}, 173-181$

Pröbster L (1993):

Survival rate of In-Ceram-restorations.

Int J Prosthodont $\underline{6}, 259-263$

Pröbster L (1996):

Four year clinical study of glass-infiltrated, sintered aluminia crowns.

J Oral Rehabil $\underline{23}, 147-151$

Pröbster L (1997):

Klinische Langzeitergebnisse mit vollkeramischen Kronen aus In-Ceram.

Quintessenz $\underline{48 / 12}, 1639-1646$

Pröbster L, Groten M:

VITA Vollkeramik - VITA In-Ceram - Leitfaden für vollkeramische Restaurationen in der zahnärztlichen Praxis. 3. Auflage,VITA,Bad Säckingen 2006,S.9-10

Rammelsberg P, Ohlmann B: Kronen- und Brückenprothetik. In: Zahnärztliche Prothetik. Gernet W, Biffar R, Schwenzer N, Ehrenfeld M 3.vollständig überarbeitete und erweiterte Auflage, Thieme-Verlag, Stuttgart 2007,S.37-39

Reitemeier B, Hansel K, Kastner C, Walter MH (2006):

Metal-ceramic failure in noble metal crowns: 7-year results of a prospective clinical trial in private practices.

Int J Prosthodont 19,397-399 
Rieger W (1993):

Aluminium- und Zirkonoxidkeramik in der Medizin.

Industrie- Diamanten- Rundschau 2,116

Roediger M, Gersdorff N, Huels A, Rinke S (2010):

Prospective evaluation of zirconia posterior fixed partial dentures: four-year clinical results.

Int J Prosthodont 23, 141-148

Rüde M, Evans AE (1989):

High toughness ceramics and ceramic composits.

Prog Mat $\underline{33}, 85$

Sadoun M:

All-ceramic bridges with slipping casting technique.

Vortrag, $7^{\text {th }}$ Internation Symposium of Ceramics, Paris 1988

Sailer I, Fehér A, Filser F, Gauckler LJ, Lüthy H, Hämmerle CH (2007):

Five-year clinical results of zirconia frameworks for posterior fixed partial dentures. Int J Prosthodont 20,383-388

Schmitter M, Rammelsberg P (2009):

Untersuchungen zum Chipping-Verhalten von 4 verschiedenen Verblendmaterialien. Interner Forschungsbericht DeguDent. Anwenderbrief Cercon 1/2009

Schneider F, Siebert F, Setz J, Boeckler A (2010):

Verblendfreie CAD/CAM-gefertigte Zirkoniumdioxidrestaurationen.

Quintessenz $\underline{61}, 47-59$

Schüller KH, Hennicken HW (1985):

Zur Systematik der keramischen Werkstoffkunde.

Ceram Forum Int 6/7,259 
Schwickerath H (1987)

Werkstoffkundliche Bewertung keramischer Kronen.

Zahnärztl Mitt 21,2422-2427

Schwickerath H, Coca I (1987):

Single crowns of glass-ceramic.

Phillip J Restaur Zahnmed 4,336-338

Scotti R, Catapano S, D`Elia A (1995):

A clinical evaluation of In-Ceram crowns.

Int J Prosthodont $\underline{8}, 320-323$

Seghi RR, Daher T, Caputo A (1990):

Relative flexural strenght of dental restorative ceramics.

Dent Mater 6(3), 181-184

Sjogren G, Lantto R, Tillberg A (1999):

Clinical evaluation of all-ceramic crowns (Dicor) in general practice.

J Prosthet Dent $\underline{81,277-284}$

Smales RJ, Hawthorne WS (1997):

Long-term survival of extensive amalgams and posterior crowns.

J Dent $\underline{25}, 225-227$

Strub JR, Türb JC, Witkowski S, Hürzeler MB, Kern M:

Curriculum Prothetik Band 3. 2. überarbeitete Auflage

Quintessenz Verlag,Berlin 1999,S.879

Swain MV (2009):

Unstable cracking (chipping) of veneering porcelain on all-ceramic dental crowns and fixed partial dentures.

Acta Biomater 5, 1668-1677 
Tinschert J, Natt G, Doose B, Fischer H, Marx R (1999):

Seitenzahnbrücken aus hochfester Strukturkeramik.

Dtsch Zahnärztl Z $\underline{54,545-550}$

Tinschert J, Natt G, Latzke P, Schulze KA, Heussen N, Spiekermann H (2007):

5-Jahres-Ergebnisse - Bewährung von vollkeramischen Brücken aus DC-Zirkon. ZWR $\underline{116}, 58$

Vult von Steyern P, Carlson P, Nilner K (2005):

All-ceramic fixed partial dentures designed according to the DC-Zirkon technique. A 2-year clinical study.

J Oral Rehabil 32,180-187.

Walton TR (1999):

A 10-year longitudinal study of fixed prosthodontics: clinical characteristics and outcome of single-unit metal-ceramic crowns.

Int J Prosthodont $\underline{6}, 519-526$

Weber T:

Memorix Zahnmedizin, Erstauflage, Thieme-Verlag, Stuttgart 1999,S.213

Wegner PK, Freitag S, Kern M (2006):

Survival rate of endodontically treated teeth with posts after prosthetic restoration. J Endod 32(10),928-931

Yeo IS, Yang JH, Lee JB (2003):

In vitro marginal fit of three all-ceramic crown systems.

J Prosthet Dent 90(5),459-464 
$9 \quad$ Anhang

9.1 Protokoll für die Nachuntersuchung (CRF)

Protokoll für die Langzeit-Nachevaluation von vollkeramischem Zahnersatz (In-Ceram)

Patientenname:

Geburtsdatum:

$\underline{\text { Restaurationen: }}$

Eingliederungsdatum:

1.

2.

3.

4.

5.

Zementierung: $\quad$ Produkt:

$\bigcirc \quad$ Adhäsiv

$\bigcirc \quad$ Konventionell

Mundhygienestatus:

Sehr Gut

○ Gut

$\bigcirc \quad$ Befriedigend

$\bigcirc \quad$ Unzureichend

O Schlecht

$\underline{\text { Restaurationen in situ: }}$

$\bigcirc \quad$ Restauration ist unverändert in situ

$\bigcirc \quad$ Restauration hat sich gelockert Zahn:

Wann:

$\bigcirc \quad$ Restauration nicht mehr funktionstüchtig und muss entfernt werden

$\bigcirc \quad$ Restauration nicht mehr in situ, Entfernungsdatum :

$\underline{\text { Vitalitätsverlust (nur ausfüllen bei negativer Vitalität mit positivem Ausgangsbefund) }}$

$\bigcirc \quad$ Apikaler Befund im Röntgenbild

$\bigcirc \quad$ Perkussionsempfindlichkeit

$\bigcirc \quad$ Endobehandlung notwendig

$\bigcirc \quad$ Endobehandlung bereits erfolgt (Zahn, Datum) : 


\section{$\underline{\text { Randschlussqualität: }}$}

$\bigcirc \quad$ Kein tastbarer Übergang zwischen Restauration und Zahn

$\bigcirc \quad$ Tastbarer Randspalt an weniger als 50\% des Umfangs der Restauration Zähne:

$\bigcirc \quad$ Tastbarer Randspalt an mehr als $50 \%$ des Umfanges der Restauration Zähne:

$\bigcirc \quad$ Tastbarer kontinuirlicher Randspalt an der gesamten Restauration Zähne:

\section{$\underline{\text { Sekundärkaries / Randverfärbungen: }}$}

$\bigcirc \quad$ Keine Verfärbung zwischen Restauration und Zahn

$\bigcirc \quad$ Lokale Verfärbungen zwischen Restauration und Zahn

$\bigcirc \quad$ Kontinuirliche Verfärbungen zwischen Restauration und Zahn

$\bigcirc \quad$ Kavitätenbildung mit erweichter Zahnhartsubstanz an folgenden Zähnen:

\section{Approximale Kontakte:}

$\bigcirc$ Kontakte optimal gestaltet

Mesialer KP etwas zu schwach Zähne:

O Mesialer KP etwas zu stark Zähne:

O Distaler KP etwas zu schwach Zähne:

○ Distaler KP etwas zu stark Zähne: $\bigcirc$ Mesialer KP fehlt: Zähne:

$\bigcirc$ Mesialer KP mit Zahnseide nicht gängig Zähne:

○ Distaler KP fehlt Zähne

$\bigcirc$ Distaler KP mit Zahnseide nicht gängig Zähne:

\section{Okklusale Kontakte:}

Optimale okklusale Abstützung der Restauration

$\bigcirc \quad$ Restauration ist geringgradig zu hoch Zähne:

$\bigcirc \quad$ Restauration hat etwas zu schwache okklusale Kontaktpunkte Zähne:

○ Lokale Schlifffacetten am Zahn Zähne:

$\bigcirc \quad$ Lokale Schlifffacetten am Antagonisten Zähne:

$\bigcirc \quad$ Starke Abrasionszeichen an der Restauration Zähne:

$\bigcirc \quad$ Starke Abrasionszeichen an den Antagonisten Zähne: 
$\bigcirc \quad$ Restauration verursacht eine klinisch manifeste Funktionsstörung Zähne:

Frakturanfälligkeit:

$\bigcirc \quad$ Kein Defekt

$\bigcirc \quad$ Riss oder Sprung

Fraktur, Zahn:

Riss oder Sprung in der Verblendkeramik, Zahn:

O Fraktur in der Verblendkeramik, Zahn:

$\bigcirc \quad$ Riss oder Sprung im Gerüst, Zahn:

O Fraktur im Gerüst, Zahn:

Sonstiges:

Untersucher:

Datum: $\quad$ Unterschrift: 


\subsection{Votum der Ethikkommission}

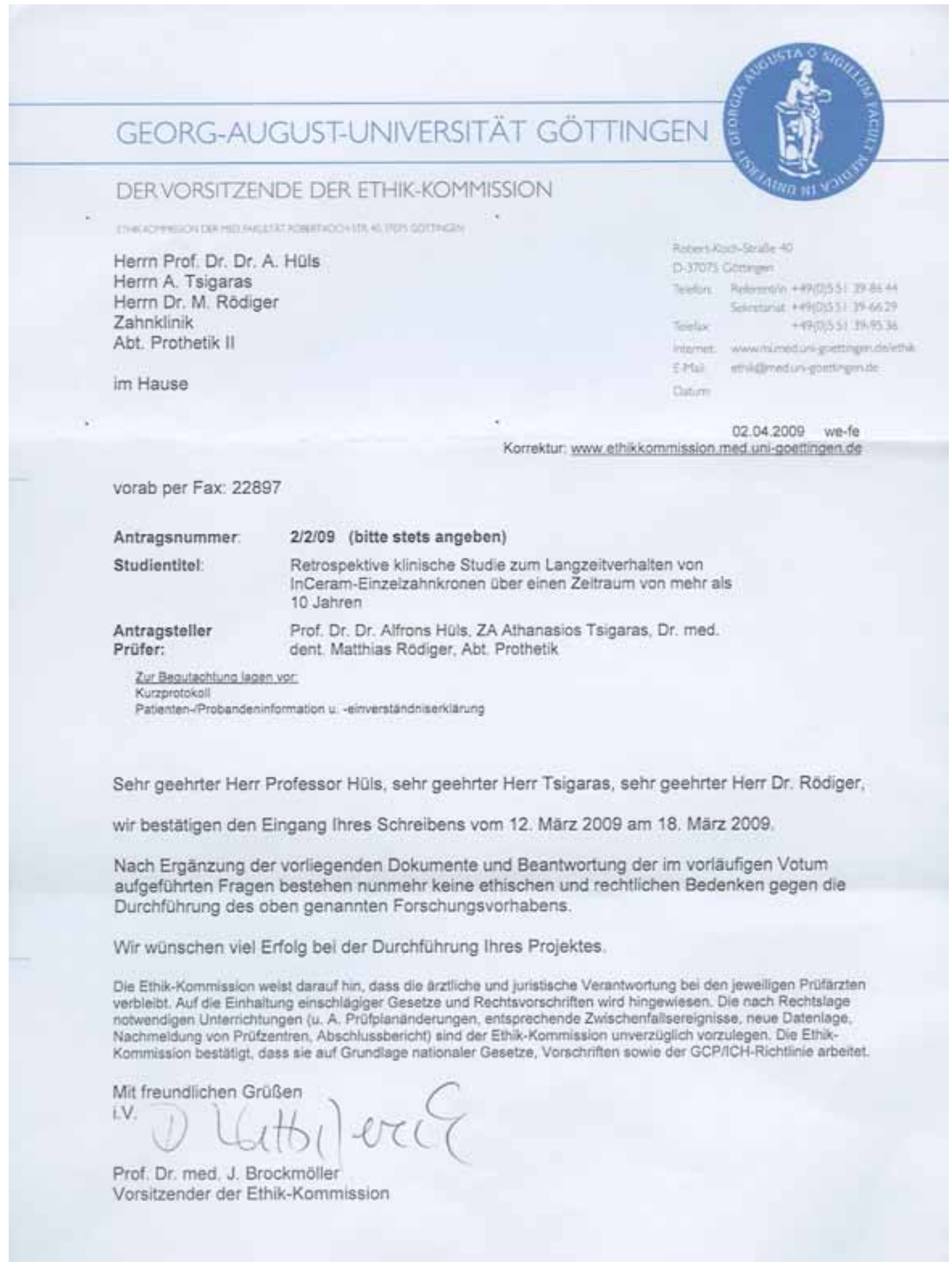




\subsection{Aufklärungsbogen Patient}

Studientitel: Retrospektive klinische Studie zum Langzeitverhalten von InCeramEinzelzahnkronen über einen Zeitraum von mehr als 10 Jahren

\section{Patientenaufklärung}

Sehr geehrte(r) Patient(in)!

Die prothetische Restauration von Zähnen mit vollkeramischen Versorgungen hat in den vergangenen Jahren immer mehr zugenommen. Die Vorteile liegen hier zum einen in der sehr guten Biokompatibilität dieser Werkstoffe und zum anderen in der zu erreichenden ästhetischen Qualität.

Die alternative Versorgung aus Metall bzw. Metall-Keramik ist allerdings schon länger im klinischen Einsatz und entsprechend sind Erfahrungen über das Langzeitverhalten von mehr als 10 Jahren für diese Restaurationen besser dokumentiert. Die bisher verfügbaren Daten für vollkeramische Versorgungen suggerieren ähnliche Erfolgswahrscheinlichkeiten, jedoch sind wissenschaftlich dokumentierte klinische Langzeitstudien für die Bewertung dieser Therapieform von großer Bedeutung, sowohl für den Zahnarzt als natürlich auch für den Patient.

Im Rahmen dieser klinischen Nachuntersuchung von vollkeramischen Kronen, die Sie in unserem Hause erhalten haben, soll die Erfolgswahrscheinlichkeit dieser Versorgung bei Verweildauern von mehr als 10 Jahren erfasst und bewertet werden. Mit Ihrer Teilnahme können Sie uns helfen, entsprechend fundierte Ergebnisse zu erhalten.

Während der Untersuchung werden verschiedene Parameter zur Bewertung erfasst: kam es zwischenzeitlich zu einem Verlust der Restauration, wo lagen ggf. die Gründe dafür, kam es zu einer Lockerung der Krone(n), sind die Pfeilerzähne vital, ist eine Karies aufgetreten, kam es zu Schäden an der Gerüst- oder der Verblendkeramik?

Die Untersuchung wird von einem Zahnarzt der Abteilung Prothetik der Universitätszahnklinik durchgeführt, ist vollkommen schmerzfrei und dauert nicht länger als 15 Minuten. Es entstehen keine Kosten für Sie. Risiken und Nebenwirkungen sind nicht zu erwarten bzw. bestehen nicht, da keinerlei Medikamente, operative Eingriffe oder Röntgenaufnahmen notwendig sind.

Ihre personenbezogenen Daten unterliegen dem Datenschutz und werden vom Leiter der Prüfung nicht weitergegeben. Sie werden pseudonymisiert behandelt, nur zu Untersuchungszwecken verwendet und nach der Auswertung vernichtet. 
Wir bitten um die freiwillige Teilnahme an der Studie. Sie können jederzeit die Teilnahme widerrufen, ohne Angabe von Gründen und ohne Nachteile erwarten zu müssen. Nach Ihrem Widerruf erfolgt unverzüglich die Vernichtung Ihrer personenbezogenen Daten.

Für Rückfragen stehen Ihnen der Studienleiter und der durchführende Zahnarzt der Abteilung Prothetik unter den oben aufgeführten Telefonnummern zur Verfügung.

Vielen Dank für Ihre Teilnahme.

Göttingen, den

Unterschrift: 


\subsection{Einverständniserklärung Patient}

Studientitel: Retrospektive klinische Studie zum Langzeitverhalten von InCeramEinzelzahnkronen über einen Zeitraum von mehr als 10 Jahren

\section{Einwilligungserklärung}

Ich, wurde von meinem Arzt vollständig über Wesen, Bedeutung und Tragweite der klinischen Untersuchung mit dem Titel:

„Retrospektive klinische Studie zum Langzeitverhalten von InCeram-Einzelzahnkronen über einen Zeitraum von mehr als 10 Jahren“

aufgeklärt.

Ich habe den Aufklärungstext gelesen und verstanden. Ich hatte die Möglichkeit, Fragen zu stellen, und habe die Antworten verstanden und akzeptiere diese. Mein Arzt hat mich über die mit der Teilnahme an der Studie verbundenen Risiken und den möglichen Nutzen informiert.

Mir ist bekannt, dass bei dieser Untersuchung personenbezogene Daten, insbesondere der medizinische Befund über mich erhoben, gespeichert und ausgewertet werden sollen. Die Verwendung der Angaben über meine Gesundheit erfolgt nach gesetzlichen Bestimmungen und setzt vor der Teilnahme an der Klinischen Prüfung die folgende freiwillig abgegebene Einwilligungserklärung voraus. Ich hatte ausreichend Zeit, mich zur Teilnahe an dieser Untersuchung zu entscheiden und weiß, dass die Teilnahme freiwillig ist.

Mir ist bekannt, dass ich jederzeit und ohne Angaben von Gründen diese Zustimmung widerrufen kann, ohne dass sich dieser Entschluss nachteilig auswirkt.

Ich habe eine Kopie der Patienteninformation und dieser Einwilligungserklärung erhalten.

Ich erkläre hiermit meine freiwillige Teilnahme an dieser Studie.

Ort und Datum

Unterschrift des Teilnehmers

Ort und Datum

Unterschrift des Prüfarztes 
9.5 Publikationen im Zusammenhang mit dieser Dissertation

9.5.1 Postervortrag:

Jahrestagung der Deutschen Gesellschaft für Zahnärztliche Prothetik und

Werkstoffkunde vom 04. - 07. November 2009 in München.

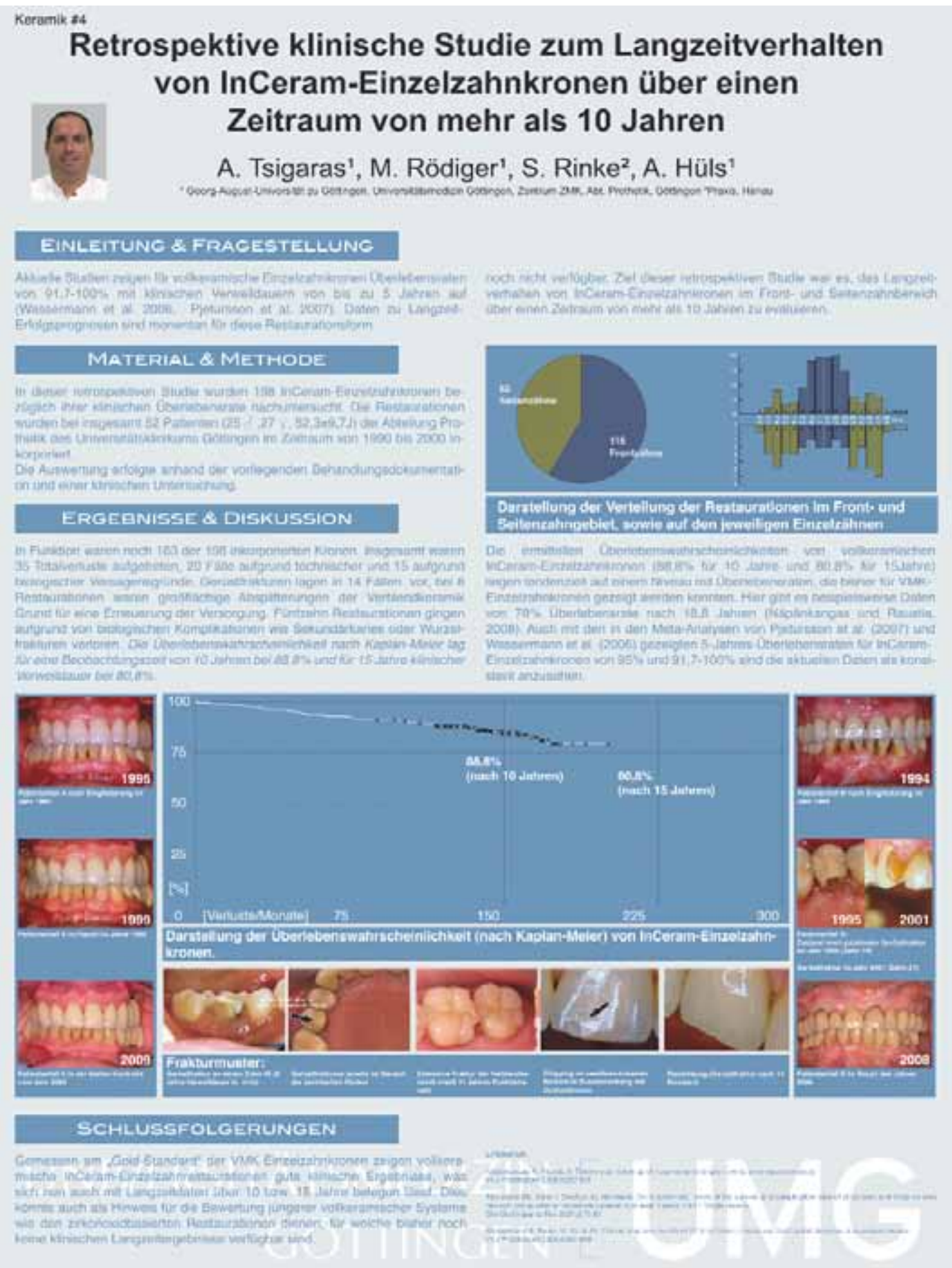

9.5.2 Wissenschaftliches Journal:

Rinke S, Roediger M, Tsigaras A, Huels A.:

Retrospective evaluation of glass-infiltrated alumina crowns up to 18 years

$\rightarrow$ J Prosthet Dent, Reviewprozess 


\section{Danksagung}

Meinem Chef, Prof. Dr. Dr. Alfons Hüls, danke ich für die freundliche Überlassung des

Themas dieser Arbeit sowie für die fachliche Unterstützung während der Bearbeitung.

Meinem Kollegen Dr. Matthias Rödiger danke ich für die außerordentlich gute Betreuung der Arbeit.

Herrn Dr. Sven Rinke möchte ich für die Unterstützung bei der Erstellung dieser Arbeit, vor allem bei der statistischen Auswertung, danken.

Schließlich möchte ich mich bei meinen Kollegen und meiner Familie recht herzlich für die Unterstützung bedanken. 


\section{Lebenslauf}

Geboren wurde ich am 10. August 1977 in Bielefeld als Sohn von Athena Tsigaras, geb. Sidiropoulou und von Dimitrios Tsigaras.

Von 1984 bis 1989 besuchte ich die griechische Grundschule in Bielefeld, von 1989 bis 1992 das griechische Gymnasium in Bielefeld, von 1992-1995 das griechische Lyzeum in Bielefeld und anschließend nahm ich an den Panhellenischen Prüfungen in Griechenland teil, die zu einem Studium in Deutschland berechtigen.

Von 1996-2000 studierte ich an der Ludwig-Maximilian-Universität München Humanmedizin. Im Anschluss begann ich mein Studium der Zahnheilkunde an der Georg-August-Universität zu Göttingen im April 2001. Die naturwissenschaftliche Vorprüfung absolvierte ich im März 2001, die zahnärztliche Vorprüfung im September des Jahres 2003.

Meine Dissertation begann ich im September 2007 bei Herrn Prof. Dr. Dr. A. Hüls in der Abteilung Prothetik des Zentrums für Zahn-, Mund- und Kieferheilkunde.

Im Dezember 2006 beendete ich an der Georg-August-Universität mein Staatsexamen erfolgreich. Seit April 2007 bin ich als wissenschaftlicher Mitarbeiter der Abteilung Prothetik des Universitätsklinikums Göttingen beschäftigt. 